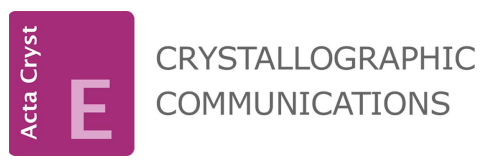

ISSN 2056-9890

Received 14 March 2015

Accepted 18 March 2015

Edited by W. T. A. Harrison, University of Aberdeen, Scotland

Keywords: crystal structure; clozapinium; crystal structure; molecular configuration hydrogen bonding; supramolecular assembly

CCDC references: $1054724 ; 1054723$; 1054722

Supporting information: this article has supporting information at journals.iucr.org/e

\section{The crystal structures of three clozapinium salts: different molecular configurations, and supramolecular assembly in one, two and three dimensions}

\author{
Manpreet Kaur, ${ }^{a}$ Jerry P. Jasinski, ${ }^{b *}$ Hemmige S. Yathirajan, ${ }^{a}$ Channappa N. \\ Kavitha $^{\mathrm{a}}$ and Christopher Glidewell ${ }^{\mathrm{C}}$ \\ ${ }^{\mathbf{a}}$ Department of Studies in Chemistry, University of Mysore, Manasagangotri, Mysore 570 006, India, ${ }^{\mathbf{b}}$ Department of \\ Chemistry, Keene State College, 229 Main Street, Keene, NH 03435-2001, USA, and 'School of Chemistry, University of \\ St Andrews, St Andrews, Fife KY16 9ST, Scotland. *Correspondence e-mail: jjasinski@keene.edu
}

The structures of three salts derived from clozapine, 8-chloro-11-(4-methylpiperazin-1-yl)-5H-dibenzo[ $b, e][1,4]$ diazepine, are reported, namely, clozapinium 3,5-dinitrobenzoate dimethyl sulfoxide monosolvate, $\mathrm{C}_{18} \mathrm{H}_{20} \mathrm{ClN}_{4}{ }^{+}$.$\mathrm{C}_{7} \mathrm{H}_{3} \mathrm{~N}_{2} \mathrm{O}_{6}{ }^{-} \cdot \mathrm{C}_{2} \mathrm{H}_{6} \mathrm{OS}$, (I), where the dimethyl sulfoxide component is disordered over two sets of atomic sites having occupancies 0.627 (2) and 0.373 (2); clozapinium hydrogen maleate 0.21 -hydrate, $\mathrm{C}_{18} \mathrm{H}_{20} \mathrm{ClN}_{4}{ }^{+} \cdot \mathrm{C}_{4} \mathrm{H}_{3} \mathrm{O}_{4}{ }^{-} \cdot 0.21 \mathrm{H}_{2} \mathrm{O}$, (II), and clozapinium 2-hydroxybenzoate, $\mathrm{C}_{18} \mathrm{H}_{20} \mathrm{ClN}_{4}{ }^{+} \cdot \mathrm{C}_{7} \mathrm{H}_{5} \mathrm{O}_{3}{ }^{-}$, (III). In all three salts, the protonation site is the methylated $\mathrm{N}$ atom of the piperazine ring, and the dimensions and conformations of the fused tricyclic system are very similar. However, differences are apparent in the piperazine component: in both compounds (II) and (III), the unprotonated $\mathrm{N}$ atom of this ring has a pyramidal geometry, but in compound (I) this atom has a planar geometry. In compound (III), both $\mathrm{N}$-substituents in this ring occupy equatorial sites, but in compound (II) the fused tricyclic system occupies an axial site of the piperazine ring. The independent components of compound (I) are linked within the selected asymmetric unit by a combination of $\mathrm{N}-\mathrm{H} \cdots \mathrm{O}$ and $\mathrm{C}-\mathrm{H} \cdots \mathrm{O}$ hydrogen bonds, and these hydrogen-bonded aggregates are linked into chains by an aromatic $\pi$ $\pi$ stacking interaction. In compound (II), the components are linked into sheets by a combination of $\mathrm{O}-\mathrm{H} \cdots \mathrm{O}, \mathrm{N}-\mathrm{H} \cdots \mathrm{O}$ and $\mathrm{C}-\mathrm{H} \cdots \mathrm{O}$ hydrogen bonds, and in compound (III), a combination of $\mathrm{N}-\mathrm{H} \cdots \mathrm{O}, \mathrm{C}-\mathrm{H} \cdots \mathrm{O}$ and $\mathrm{C}-\mathrm{H} \cdots \mathrm{N}$ hydrogen bonds links the components into a three-dimensional framework structure. Comparisons are made with some similar compounds.

\section{Chemical context}

Clozapine, 8-chloro-11-(4-methylpiperazin-1-yl)-5H-dibenzo$[b, e][1,4]$ diazepine, is a well established medication used in the treatment of schizophrenia which in general leads to a lower incidence of adverse side effects such as Parkinsonian-type symptoms than some other treatments (Breier et al., 1994). Although structures have been reported for both the free base itself (Petcher \& Weber, 1976) and for its doubly-protonated di-cation, as the dibromide salt (Fillers \& Hawkinson, 1982), there appear to be no reports of the structures of any monoprotonated clozapine derivatives. Accordingly, we have now determined the structures of three such salts with a variety of counter-ions. Of these salts, the 3,5-dinitrobenzoate crystallizes from dimethyl sulfoxide as a stoichiometric monosolvate (I) (Fig. 1, Scheme 1); however, the hydrogen maleate crystallizes from the same solvent as a partial hydrate (II) (Fig. 2); 
and the 2-hydroxybenzoate crystallizes from a 1:1 mixture of acetonitrile and methanol in a solvent-free form (III) (Fig. 3). A number of other such salts were prepared, but no crystals suitable for single-crystal X-ray diffraction have so far been obtained from these, despite attempts to prepare crystals using a range of solvents. The aims of the present study are firstly to establish the site of protonation in the mono-protonated cations; secondly, to compare the conformations of the clozapinium cations; and thirdly, to explore the supramolecular assembly in these three salts.<smiles>CS(=O)(=O)[N+]1CCN(C2=Nc3cc(Cl)ccc3Nc3ccccc32)CC1</smiles><smiles>O=[N+]([O-])c1cc([N+](=O)[O-])cc([N+](=O)[O-])c1</smiles>

Me<smiles>C[N+]1(C)CCN(C2=Nc3cc(Cl)ccc3Nc3ccccc32)CC1</smiles>

(I)<smiles>O=C(O)/C=C\C(=O)O</smiles>
$0.21\left(\mathrm{H}_{2} \mathrm{O}\right)$

(II)<smiles>CS(=O)(=O)[N+]1CCN(C2=Nc3cc(Cl)ccc3Nc3ccccc32)CC1</smiles><smiles>O=C(O)c1ccccc1O</smiles>

(III)

\section{Structural commentary}

Compound (I) consists of a clozapinium cation, in which protonation has occurred at the protonated $\mathrm{N}$ atom of the piperazine ring, as is also observed in both of compounds (II) and (III), a 3,5-dinitrobenzoate anion, and a molecule of dimethyl sulfoxide (DMSO), which is disordered over two orientations having site occupancies of 0.627 (2) and 0.273 (2), respectively (Fig. 1). It was possible to select an asymmetric unit for (I) in which the three components are linked by $\mathrm{N}-$ $\mathrm{H} \cdots \mathrm{O}$ hydrogen bonds (Fig. 1, Table 1). The $\mathrm{N}-\mathrm{H} \cdots \mathrm{O}$ hydrogen bond between the two ionic components within the

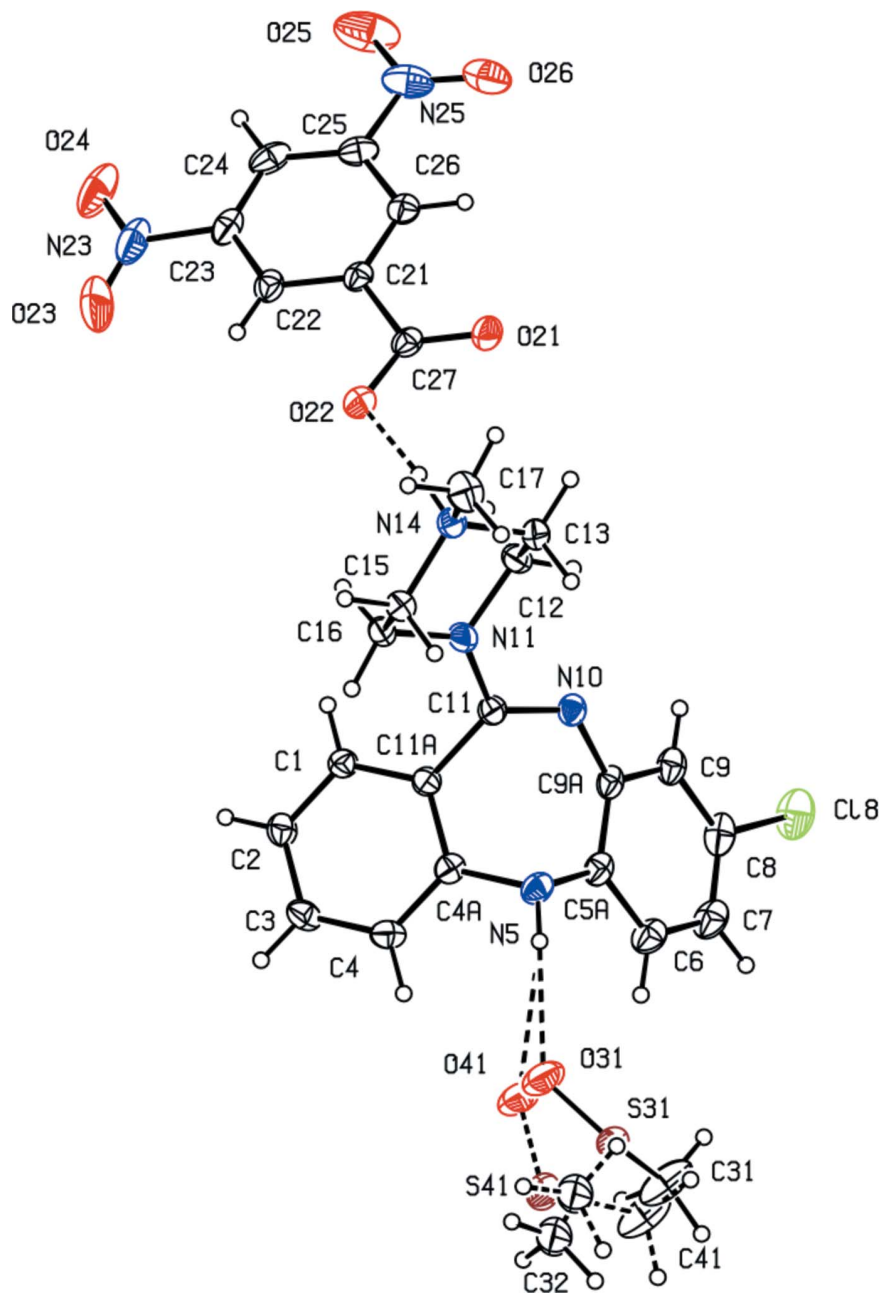

Figure 1

The independent components of compound (I), showing the atomlabelling scheme and the $\mathrm{N}-\mathrm{H} \cdots \mathrm{O}$ hydrogen bonds within the selected asymmetric unit. Displacement ellipsoids are drawn at the $30 \%$ probability level and the major and minor orientations of the disordered dimethyl sulfoxide component, containing atoms S31 and S41, respectively, have occupancies 0.627 (2) and 0.373 (2).

selected asymmetric unit is a charge-assisted hydrogen bond (Gilli et al., 1994) and it is nearly linear with fairly short $\mathrm{H} \cdots \mathrm{O}$ and $\mathrm{N}$... O distances (Table 1). There are also some short $\mathrm{C}-$ $\mathrm{H}$. . O contacts between the cation and the disordered DMSO components, but those to the major component, in particular, have long $\mathrm{H} \cdots \mathrm{O}$ distances, and $\mathrm{C}-\mathrm{H} \cdots \mathrm{O}$ angles which are less than $140^{\circ}$ (cf. Wood et al., 2009) so these may be better regarded as adventitious contacts rather than structurally significant interactions.

Compound (II) consists of a clozapinium cation, a hydrogen maleate anion which contains a short intra-anion $\mathrm{O}-\mathrm{H} \cdots \mathrm{O}$ hydrogen bond (Table 1), and a partial occupancy water molecule whose refined site occupancy is 0.210 (7): despite the short $\mathrm{O} \cdots \mathrm{O}$ distance in the intra-anion hydrogen bond, the $\mathrm{H}$ atom is significantly displaced from a position equidistant from the two $\mathrm{O}$ atoms involved (Table 1). The two ionic components are linked by a nearly linear charge-assisted hydrogen bond (Fig. 2), while the partially occupied water site forms hydrogen bonds to two anions (Table 1). 
Table 1

Hydrogen bonds and short intramolecular contacts $\left(\AA{ }^{\circ}\right)$ for compounds (I)-(III).

$\mathrm{Cg} 1$ and $\mathrm{Cg} 2$ represent the centroids of rings $\mathrm{C} 5 A / \mathrm{C} 6-\mathrm{C} 9 / \mathrm{C} 9 A$ and $\mathrm{C} 1-\mathrm{C} 4 /$ $\mathrm{C} 4 A / \mathrm{C} 11 A$, respectively

\begin{tabular}{|c|c|c|c|c|c|}
\hline Compound & $D-\mathrm{H} \cdots A$ & $D-\mathrm{H}$ & $\mathrm{H} \cdots A$ & $D \cdots A$ & $D-\mathrm{H} \cdots A$ \\
\hline \multirow[t]{5}{*}{ (I) } & $\mathrm{N} 5-\mathrm{H} 5 \cdots \mathrm{O} 31$ & $0.83(3)$ & $2.10(3)$ & $2.921(4)$ & $170(3)$ \\
\hline & $\mathrm{N} 14-\mathrm{H} 14 \cdots \mathrm{O} 22$ & $1.00(3)$ & $1.58(3)$ & $2.575(3)$ & $176(2)$ \\
\hline & $\mathrm{C} 4-\mathrm{H} 4 \cdots \mathrm{O} 31$ & 0.95 & 2.58 & $3.342(4)$ & 137 \\
\hline & $\mathrm{C} 4-\mathrm{H} 4 \cdots \mathrm{O} 41$ & 0.95 & 2.38 & $3.208(7)$ & 146 \\
\hline & $\mathrm{C} 6-\mathrm{H} 6 \cdots \mathrm{O} 31$ & 0.95 & 2.54 & $3.313(5)$ & 139 \\
\hline \multirow[t]{8}{*}{ (II) } & $\mathrm{N} 5-\mathrm{H} 5 \cdots \mathrm{O} 22^{\mathrm{i}}$ & $0.86(3)$ & $2.24(3)$ & $3.084(2)$ & $170(3)$ \\
\hline & $\mathrm{N} 14-\mathrm{H} 14 \cdots \mathrm{O} 23$ & $0.87(3)$ & $1.83(2)$ & $2.688(2)$ & $173(2)$ \\
\hline & $\mathrm{O} 21-\mathrm{H} 21 \cdots \mathrm{O} 24$ & $1.00(5)$ & $1.47(5)$ & $2.420(3)$ & $157(5)$ \\
\hline & $\mathrm{O} 31-\mathrm{H} 31 A \cdots \mathrm{O} 24$ & 0.90 & 2.05 & $2.913(11)$ & 160 \\
\hline & $\mathrm{O} 31-\mathrm{H} 31 B \cdots \mathrm{O} 21^{\mathrm{ii}}$ & 0.90 & 2.37 & $3.232(11)$ & 161 \\
\hline & $\mathrm{C} 12-\mathrm{H} 12 A \cdots \mathrm{O} 22^{\mathrm{iii}}$ & 0.99 & 2.48 & $3.308(2)$ & 141 \\
\hline & $\mathrm{C} 15-\mathrm{H} 15 A \cdots C g 1^{\text {iv }}$ & 0.99 & 2.95 & $3.642(2)$ & 128 \\
\hline & $\mathrm{C} 15-\mathrm{H} 15 B \cdots C g 2^{\mathrm{iv}}$ & 0.99 & 2.95 & $3.759(2)$ & 139 \\
\hline \multirow[t]{7}{*}{ (III) } & $\mathrm{N} 14-\mathrm{H} 14 \cdots \mathrm{O} 22$ & $0.89(6)$ & $1.75(6)$ & $2.612(4)$ & $164(5)$ \\
\hline & $\mathrm{O} 23-\mathrm{H} 23 \cdots \mathrm{O} 21$ & $1.01(11)$ & $1.52(10)$ & $2.507(5)$ & $161(9)$ \\
\hline & $\mathrm{C} 4-\mathrm{H} 4 \cdots \mathrm{O} 22^{\mathrm{v}}$ & 0.95 & 2.23 & $3.261(4)$ & 166 \\
\hline & $\mathrm{C} 9-\mathrm{H} 9 \cdots \mathrm{O} 22^{\mathrm{v}}$ & 0.95 & 2.25 & $3.202(4)$ & 176 \\
\hline & $\mathrm{C} 12-\mathrm{H} 12 A \cdots \mathrm{O} 21$ & 0.99 & 2.44 & $3.306(5)$ & 146 \\
\hline & $\mathrm{C} 12-\mathrm{H} 12 B \cdots \mathrm{N} 5^{\mathrm{vi}}$ & 0.99 & 2.56 & $3.539(4)$ & 170 \\
\hline & $\mathrm{C} 24-\mathrm{H} 24 \cdots C g 1^{\mathrm{vii}}$ & 0.95 & 2.83 & $3.637(5)$ & 144 \\
\hline
\end{tabular}

Symmetry codes: (i) $-1+x, \frac{3}{2}-y,-\frac{1}{2}+z$; (ii) $-x, 2-y, 1-z$; (iii) $2-x, 1-y, 1-z$; (iv) $1-x, \frac{1}{2}+y, \frac{1}{2}-z$; (v) $\frac{1}{2}+x,-\frac{1}{2}+y, z$; (vi) $x, 1-y, \frac{1}{2}+z$; (vii) $-\frac{1}{2}+x, \frac{1}{2}+y,-1+z$.

There is again an intra-anion $\mathrm{O}-\mathrm{H} \cdots \mathrm{O}$ hydrogen bond in the 2-hydroxybenzoate component of compound (III) and, again, the two ionic components are linked by a fairly short, charge-assisted hydrogen bond (Fig. 3). It is of interest to note both the general similarity in the dimensions of the two intraanion hydrogen bonds in compounds (II) and (III), and also that in each of (I)-(III), the site of the protonation of the

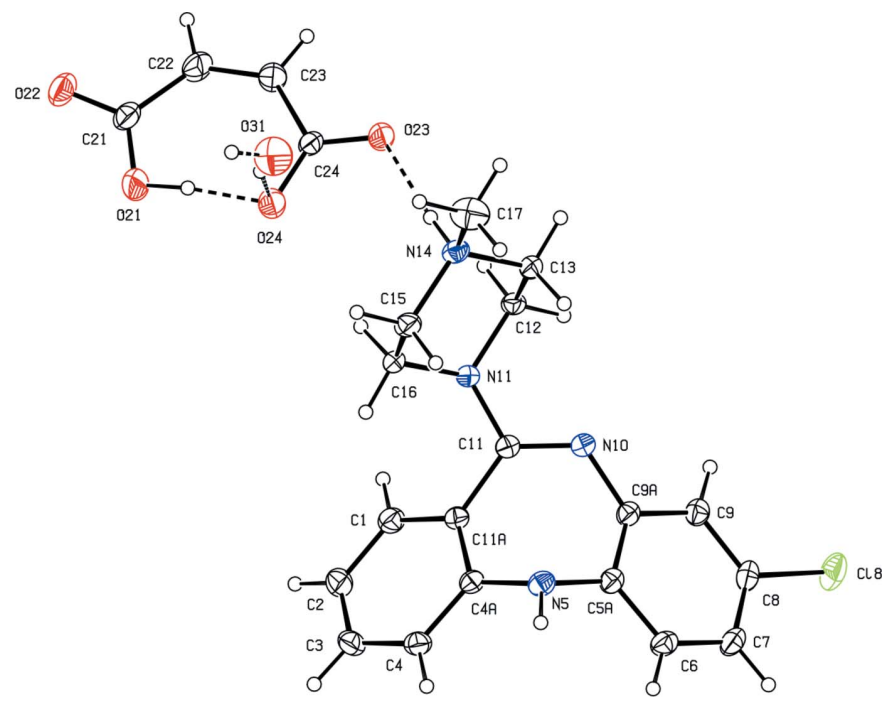

Figure 2

The independent components of compound (II), showing the atomlabelling scheme, and the $\mathrm{O}-\mathrm{H} \cdots \mathrm{O}$ and $\mathrm{N}-\mathrm{H} \cdots \mathrm{O}$ hydrogen bonds within the selected asymmetric unit. Displacement ellipsoids are drawn at the $30 \%$ probability level and the water molecule, containing atom $\mathrm{O} 31$, has occupancy $0.210(7)$.

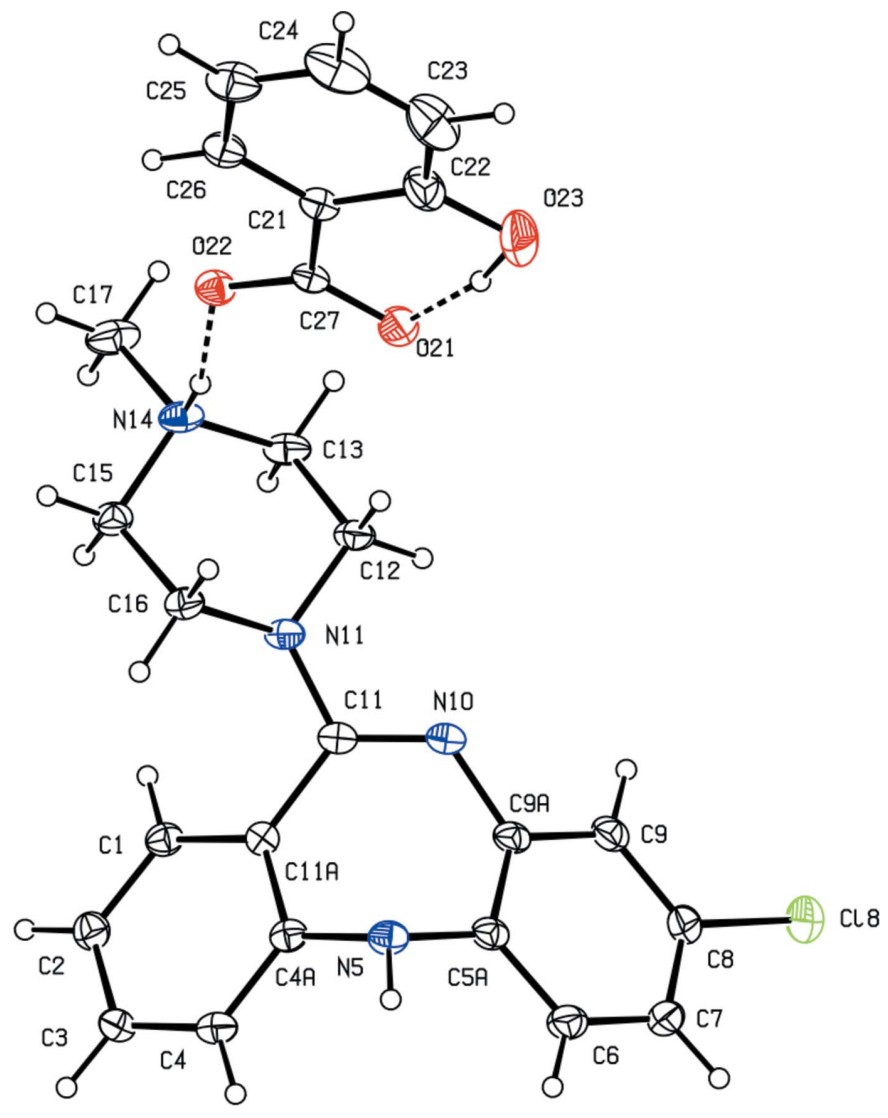

Figure 3

The independent components of compound (III), showing the atomlabelling scheme, and the $\mathrm{O}-\mathrm{H} \cdots \mathrm{O}$ and $\mathrm{N}-\mathrm{H} \cdots \mathrm{O}$ hydrogen bonds within the selected asymmetric unit. Displacement ellipsoids are drawn at the $30 \%$ probability level.

clozapine is the methylated atom N14 of the piperazine ring. In each case, this $\mathrm{N}-\mathrm{H}$ bond participates in a short chargeassisted hydrogen bond between the ionic components. As discussed below, this is the only $\mathrm{N}-\mathrm{H} \cdots \mathrm{O}$ hydrogen bond involving the ionic components in both compound (I) and compound (III).

In the clozapinium cations of compounds (I)-(III), the fused tricyclic units exhibit very similar conformations, as

Table 2

Selected geometric parameters $\left(^{\circ}\right)$ for compounds (I)-(III).

'Dihedral' denotes the dihedral angles between the mean planes of rings $\mathrm{C} 1-$ $\mathrm{C} 4 / \mathrm{C} 4 A / \mathrm{C} 11 A$ and $\mathrm{C} 5 A / \mathrm{C} 6-\mathrm{C} 9 / \mathrm{C} 9 A$.

\begin{tabular}{lccr}
\hline Parameter & $(\mathrm{I})$ & (II) & \multicolumn{1}{c}{ (III) } \\
\hline C11-N11-C12 & $120.80(19)$ & $118.45(15)$ & $118.3(3)$ \\
C11-N11-C16 & $126.01(19)$ & $122.04(15)$ & $121.7(3)$ \\
C12-N11-C16 & $112.91(18)$ & $111.09(14)$ & $111.0(3)$ \\
C4 $A-\mathrm{N} 5-\mathrm{C} 5 A-\mathrm{C} 6$ & $-117.8(2)$ & $-116.17(19)$ & $-117.1(3)$ \\
C5 $A-\mathrm{N} 5-\mathrm{C} 4 A-\mathrm{C} 4$ & $115.1(3)$ & $120.07(19))$ & $115.3(3)$ \\
C1-C11 $A-\mathrm{C} 11-\mathrm{N} 10$ & $-129.2(3)$ & $-137.5(2)$ & $-139.6(3)$ \\
C9-C9A-N10-C11 & $146.3(2)$ & $141.11(19)$ & $141.9(3)$ \\
C9 $A-\mathrm{N} 10-\mathrm{C} 11-\mathrm{C} 11 A$ & $-7.9(4)$ & $0.9(3)$ & $1.0(5)$ \\
N10-C11-N11-C12 & $6.3(3)$ & $2.7(3)$ & $5.5(4)$ \\
C11-N11-C12-C13 & $-120.8(2)$ & $-90.0(2)$ & $151.8(3)$ \\
Dihedral & $62.21(11)$ & $60.97(9)$ & $59.07(16)$ \\
\hline
\end{tabular}



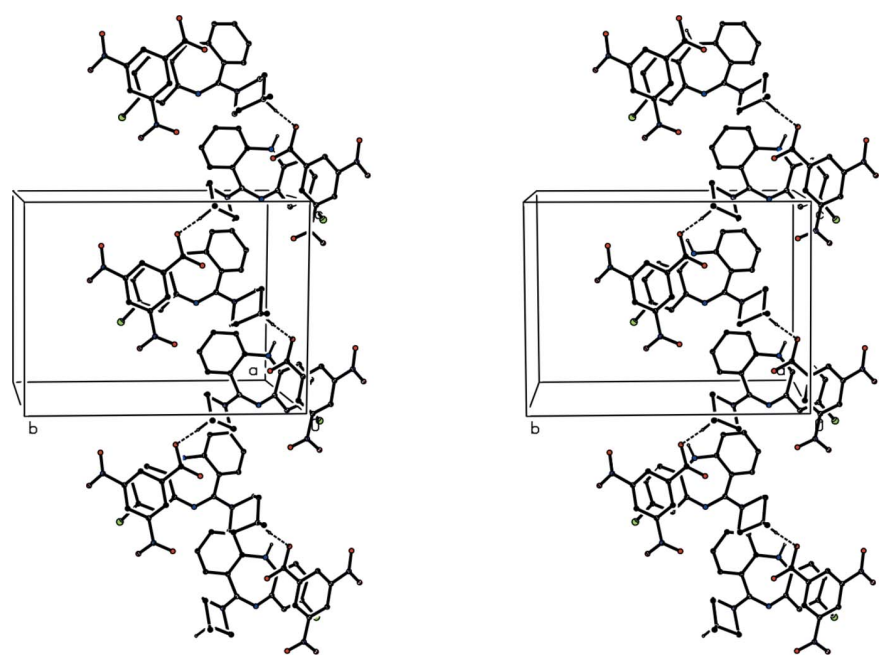

Figure 4

A stereoview of part of the crystal structure of compound (I), showing the formation of a $\pi$-stacked chain of hydrogen-bonded ion pairs. For the sake of clarity, $\mathrm{H}$ atoms not involved in the hydrogen bonds (shown as dashed lines) have been omitted, as have the disordered DMSO molecules.

shown by the relevant torsional and dihedral angles (Table 2) which define the relative orientations of the three rings. It is interesting to note that corresponding pairs of torsional angles involving either one or the other of the two aryl rings consistently have similar magnitudes but opposite signs, indicative of near mirror symmetry, provided the difference in the atomic types $\mathrm{N} 10$ and $\mathrm{C} 11$ is ignored, with the pseudo mirror containing the bond $\mathrm{N} 5-\mathrm{H} 5$ and passing through the midpoint of the bond $\mathrm{N} 10-\mathrm{C} 11$. However, there are some interesting differences between (I)-(III) in respect of the piperazine rings, which in each compound adopt a chair conformation, with protonation at the methylated atom N14, where the methyl atom $\mathrm{C} 17$ always occupies the equatorial site. While the geometry at N11 is planar within experimental uncertainty in compound (I), it is pyramidal in each of (II) and (III). In addition, the atom $\mathrm{C} 11$ (and hence the bulky tricyclic system) occupies the equatorial site at N11 in compound (III), but in compound (II) the tricyclic unit unexpectedly occupies the axial site at atom N11, as indicated by the values of the torsional angles $\mathrm{C} 11-\mathrm{N} 11-\mathrm{C} 12-\mathrm{C} 13$ for these two compounds (Table 2).

\section{Supramolecular features}

The supramolecular assembly in compounds (I)-(III) provides structures in one, two and three dimensions respectively. There are no hydrogen bonds in the structure of compound (I) other than those within the selected asymmetric unit (Table 1, Fig. 1). However, the hydrogen-bonded ionic components are linked into a chain by an aromatic $\pi-\pi$ stacking interaction. The $\mathrm{C} 5 A, \mathrm{C} 6-\mathrm{C} 9, \mathrm{C} 9 A$ ring in the cation at $(x, y, z)$ makes a dihedral angle of only $1.34(12)^{\circ}$ with the $\mathrm{C} 21-\mathrm{C} 26$ ring in the anion at $\left(x, \frac{1}{2}-y, \frac{1}{2}+z\right)$. The distance between the centroids of these two rings is 3.4583 (14) $\AA$ and the shortest perpendicular

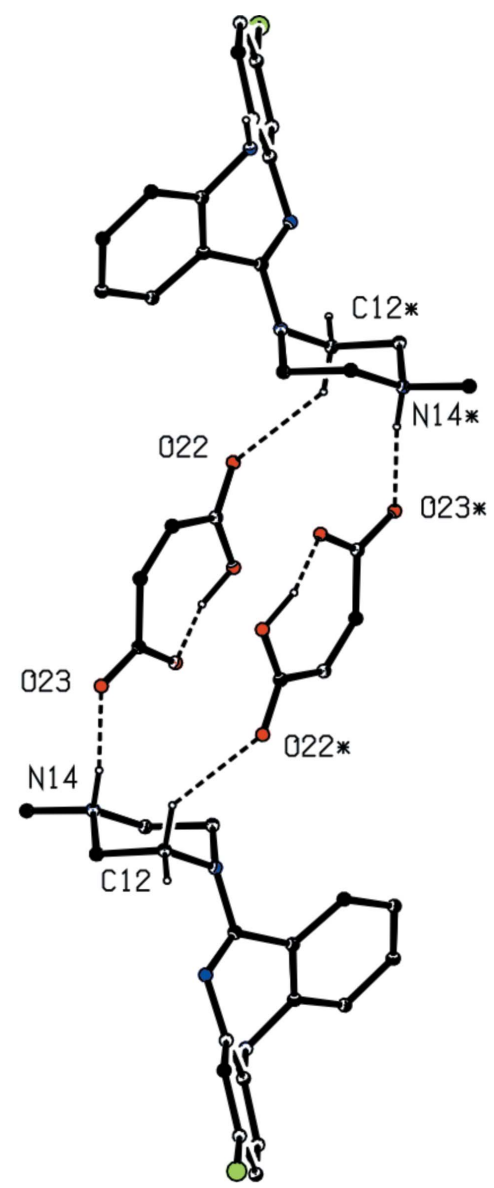

Figure 5

Part of the crystal structure of compound (II) showing the formation of a centrosymmetric four-ion aggregate. For the sake of clarity, the unit-cell outline and the $\mathrm{H}$ atoms bonded to $\mathrm{C}$ atoms not involved in the motif shown have been omitted. Atoms marked with an asterisk (*) are at the symmetry position $(2-x, 1-y, 1-z)$.

distance between the centroid of one ring and the plane of the other is 3.2761 (1) $\AA$, corresponding to a ring-centroid offset of $c a 1.11 \AA$. This stacking interaction links the hydrogenbonded ionic components into a chain running parallel to the [001] direction (Fig. 4). Two chains of this type, related to one another by inversion, pass through each unit cell, but there are no direction-specific interactions between adjacent chains. The DMSO molecules are pendent from the chains but otherwise play no part in the supramolecular assembly, so that their role may be largely that of filling otherwise empty cavities within the structure formed by the ionic components.

In compound (II) a combination of $\mathrm{O}-\mathrm{H} \cdots \mathrm{O}, \mathrm{N}-\mathrm{H} \cdots \mathrm{O}$ and $\mathrm{C}-\mathrm{H} \cdots \mathrm{O}$ hydrogen bonds (Table 1 ) links the independent components into complex sheets, but the sheet formation can readily be analysed in terms of a small number of fairly simple sub-structures (Ferguson et al., 1998a,b; Gregson et al., 2000). Ion pairs (Fig. 2) which are related by inversion are linked by $\mathrm{C}-\mathrm{H} \cdots \mathrm{O}$ hydrogen bonds, forming a cyclic centrosymmetric aggregate characterized by an $R_{4}^{4}(22)$ (Bernstein et al., 1995) motif, with the reference aggregate centred at $\left(1, \frac{1}{2}, \frac{1}{2}\right)$ (Fig. 5). In a second sub-structure, ion pairs which are related by a glide plane are linked by $\mathrm{N}-\mathrm{H} \cdots \mathrm{O}$ 

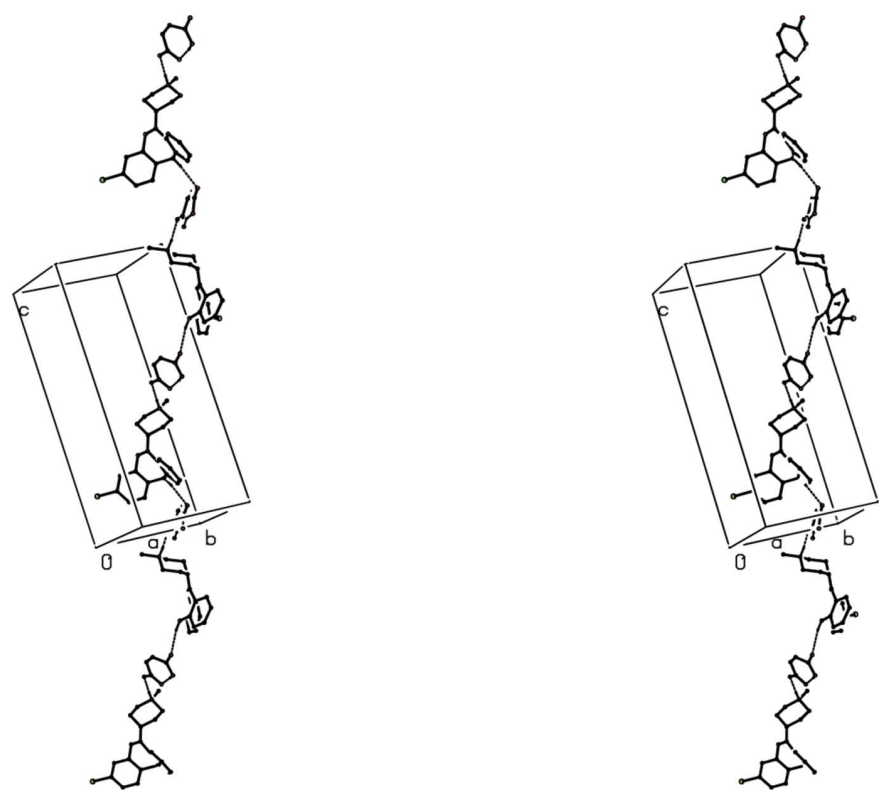

Figure 6

A stereoview of part of the crystal structure of compound (II) showing the formation of hydrogen-bonded $C_{2}^{2}(16)$ chain parallel to [201]. For the sake of clarity, $\mathrm{H}$ atoms bonded to $\mathrm{C}$ atoms and the partial-occupancy water molecules have been omitted.

hydrogen bonds to form a $C_{2}^{2}(16)$ chain running parallel to the [201] direction (Fig. 6). This chain motif directly links the reference four-ion aggregate (Fig. 5) centred at $\left(1, \frac{1}{2}, \frac{1}{2}\right)$ to the four symmetry-related aggregates centred at $(0,0,0),(0,1,0)$, $(2,0,1)$ and $(2,1,1)$, so forming a sheet lying parallel to $(10 \overline{2})$ (Fig. 7). Embedded within this sheet is a further cyclic motif, which is formally centrosymmetric, containing two anions and two water molecules. However, since the occupancy of the water sites is only 0.210 (7), if either of the two water sites in this motif is occupied there is a high probability that the other such site will be unoccupied: indeed, in the majority of cases, neither site will be occupied.
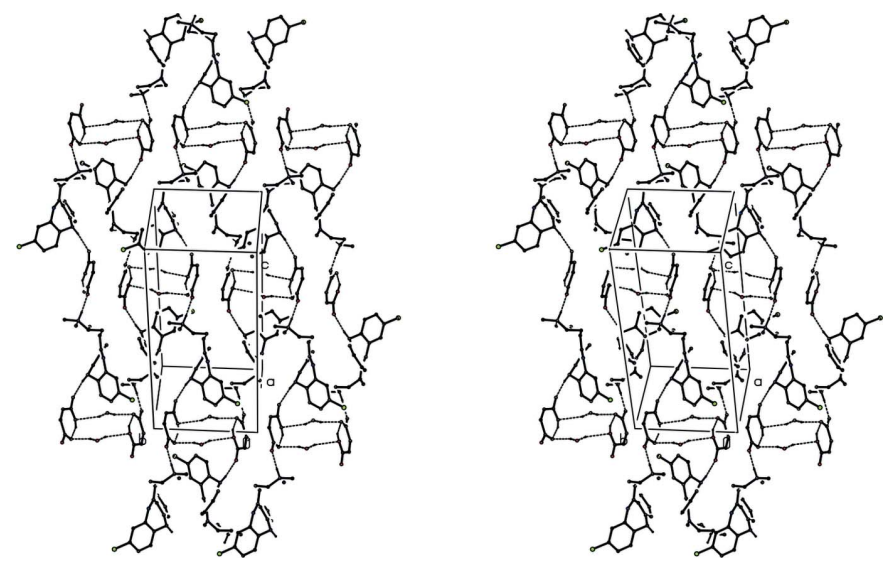

Figure 7

A stereoview of part of the crystal structure of compound (II) showing the formation of a hydrogen-bonded sheet lying parallel to $(10 \overline{2})$. For the sake of clarity, $\mathrm{H}$ atoms bonded to $\mathrm{C}$ atoms not involved in the motifs shown have been omitted.
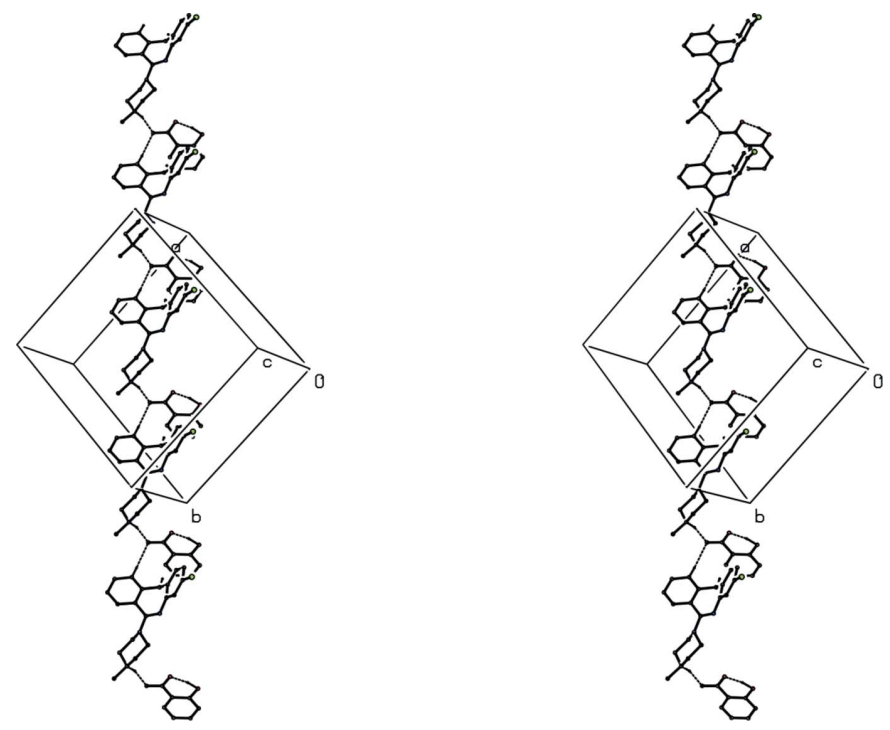

Figure 8

A stereoview of part of the crystal structure of compound (III) showing the formation of a hydrogen-bonded $C_{2}^{2}(11)$ chain running parallel to [11ํ]. For the sake of clarity, $\mathrm{H}$ atoms not involved in the motif shown have been omitted.

The independent components of compound (III) are linked into a three-dimensional framework structure by a combination of $\mathrm{N}-\mathrm{H} \cdots \mathrm{O}, \mathrm{O}-\mathrm{H} \cdots \mathrm{O}$ and $\mathrm{C}-\mathrm{H} \cdots \mathrm{N}$ hydrogen bonds (Table 1), and again the formation of the framework is most readily analysed in terms of three one-dimensional substructures. In the simplest of these sub-structures, the $\mathrm{C}-\mathrm{H} \cdots \mathrm{O}$ hydrogen bond involving atom $\mathrm{C} 4$ links ion pairs related by translation into a $C_{2}^{2}(11)$ chain running parallel to the [1 $\left.1 \overline{1} 0\right]$ direction (Fig. 8). The second sub-structure involves both $\mathrm{C}-$ $\mathrm{H} \cdots \mathrm{N}$ and $\mathrm{C}-\mathrm{H} \cdots \mathrm{O}$ hydrogen bonds: cations related by the $c$-glide plane at $y=0.5$ are linked by $\mathrm{C}-\mathrm{H} \cdots \mathrm{N}$ hydrogen bonds into $C(7)$ chains running parallel to the [001] direction, and similarly related ion pairs are linked by the $\mathrm{C}-\mathrm{H} \cdots \mathrm{O}$ hydrogen bond involving atom $\mathrm{C} 9$ to form a $C_{2}^{2}(11)$ chain also running parallel to [001], such that the combined effect of these two hydrogen bonds generates a $C(7) C_{2}^{2}(11)\left[R_{3}^{2}(19)\right]$
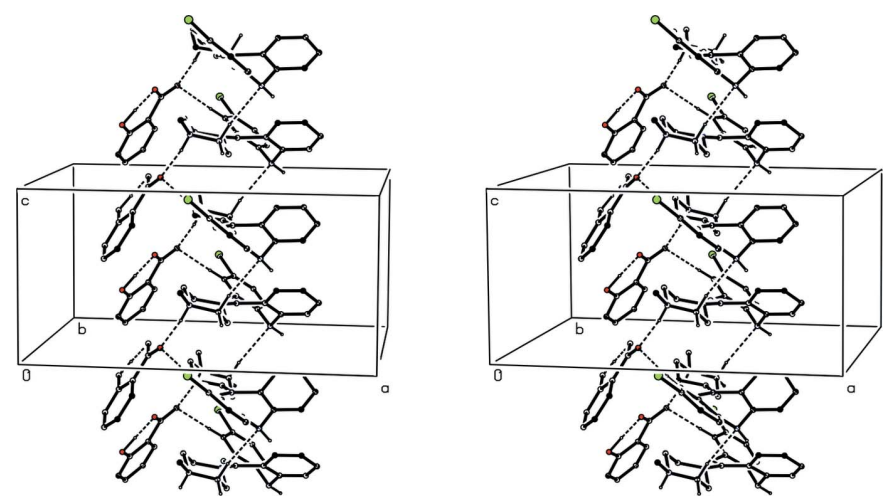

Figure 9

A stereoview of part of the crystal structure of compound (III) showing the formation of a hydrogen-bonded $C(7) C_{2}^{2}(11)\left[R_{3}^{2}(19)\right]$ chain of rings running parallel to [001]. For the sake of clarity, $\mathrm{H}$ atoms bonded to the $\mathrm{C}$ atoms not involved in the motif shown have been omitted. 

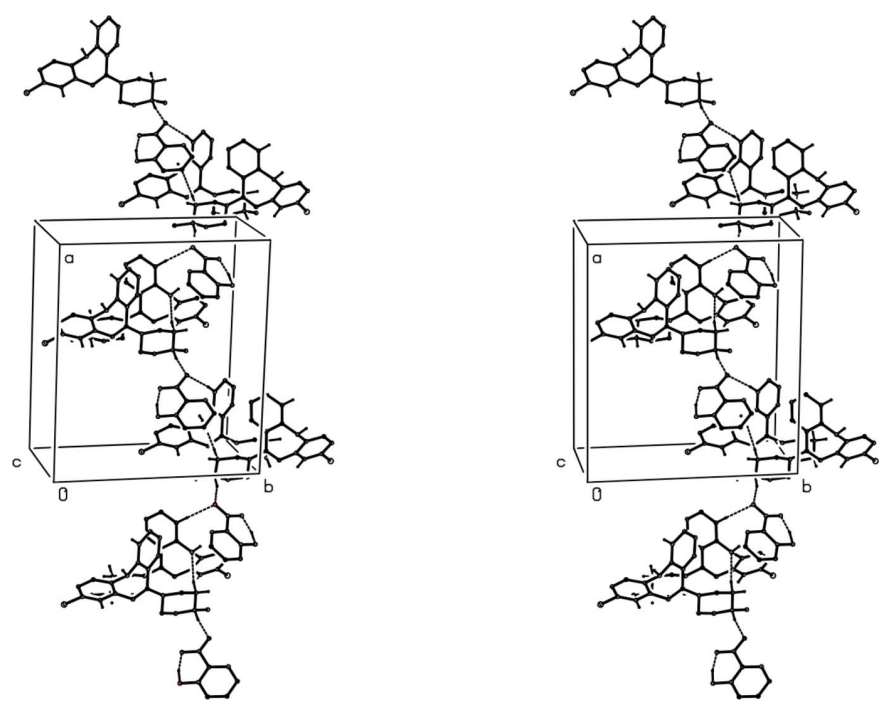

Figure 10

A stereoview of part of the crystal structure of compound (III) showing the formation of a hydrogen-bonded chain running parallel to [101]. For the sake of clarity, $\mathrm{H}$ atoms bonded to the $\mathrm{C}$ atoms not involved in the motif shown have been omitted.

chain of rings running parallel to [001] (Fig. 9). Finally, the alternating action of the hydrogen bonds involving, atom $\mathrm{C} 4$ on the one hand, and atoms C9 and C15 on the other (Table 1) generates a complex chain running parallel to the [101] direction (Fig. 10).

\section{Database survey}

It is of interest briefly to compare the structures reported here for the salts (I)-(III) with those of some closely related analogues, in particular clozapine itself, compound (IV) (see Scheme 2) and the dihydrobromide salt (V).

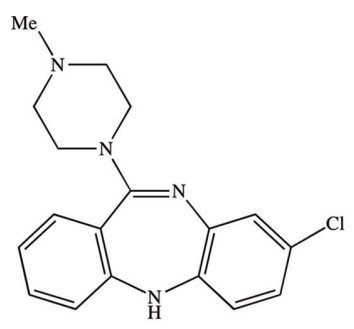

(IV)

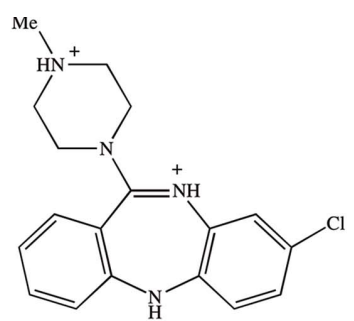

(V)

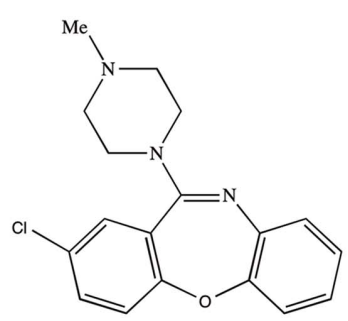

(VI)

In the free base clozapine, which crystallizes in the space group $P 2_{1} 2_{1} 2_{1}$ (Petcher \& Weber, 1976), the geometry at the piperazinyl $\mathrm{N}$ atom corresponding to atom N11 in compounds (I)-(III) is very nearly planar, with a sum of interbond angles of $357(2)^{\circ}$, and there are no direction-specific interactions between the molecules: in particular, the $\mathrm{N}-\mathrm{H}$ bond does not participate in any kind of hydrogen bond. In the salt (V), which crystallizes in space group $P 2_{1} / c$ (Fillers \& Hawkinson, 1982), the protonation sites are the $\mathrm{N}$ atoms corresponding to atoms N10 and N14 in compounds (I)-(III), so that the doubly bonded $\mathrm{N}$ atom of the diazepine ring is protonated in preference to the second $\mathrm{N}$ atom of the piperazine ring where, as in (IV), the geometry in nearly planar, with a sum of interbond angles of $357(2)^{\circ}$ : the individual ionic components in $(\mathrm{V})$ are linked by charge-assisted $\mathrm{N}-\mathrm{H} \cdots \mathrm{Br}$ hydrogen bonds, such that cations related by a $2_{1}$ screw axis are bridged by one of the two independent anions to form a $C_{2}^{1}(7)$ chain, from which the anions of the second type are pendent. Loxapine, compound (VI), is similar to clozapine but differs from it in two respects: the nature of the hetero-atoms in the seven-membered ring, and the location of the $\mathrm{Cl}$ substituent. Here again there are no direction-specific interactions between the molecules (Petcher \& Weber, 1976). The overall molecular shapes of the molecules of compounds (IV) and (VI) are extremely similar, and it was suggested (Petcher \& Weber, 1976) that the structures observed in the solid state represented a preferred form which persists in aqueous solutions and at the site of neuroleptic receptors. However, in the presence of charge-assisted hydrogen bonds in compounds (I)-(III), reported here, which are probably slightly stronger that those between the molecules of (IV) and (VI) and adjacent water molecules in solution, the molecular configurations show some significant differences, as noted above, so that no preferred configuration is apparent from the structures of (I)(III).

\section{Synthesis and crystallization}

Clozapine was a gift from $\mathrm{R}$ L Fine Chem, Bengaluru, Karnataka, India. Equimolar quantities of clozapine and the appropriate acid (1.53 mmol of each component) were dissolved in methanol at $333 \mathrm{~K}$. The solutions were permitted to cool to ambient temperature, and the resulting crystals were then collected by filtration, and dried over phosphorus(V) oxide. Crystals suitable for single-crystal X-ray diffraction were obtained by slow evaporation, at ambient temperature and in the presence of air, of solutions in dimethyl sulfoxide, for compounds (I) and (II), and a mixture (1:1 v/v) of acetonitrile and methanol for compound (III).

\section{Refinement}

Crystal data, data collection and structure refinement details are summarized in Table 3. The $\mathrm{H}$ atoms bonded to $\mathrm{C}$ or $\mathrm{N}$ atoms in the ionic components of compounds (I)-(III) were all located in difference maps. The $\mathrm{H}$ atoms bonded to $\mathrm{C}$ atoms were then treated as riding atoms in geometrically idealized positions with $\mathrm{C}-\mathrm{H}$ distances of $0.95 \AA$ (alkenyl and aromatic), $0.98 \AA\left(\mathrm{CH}_{3}\right)$ or $0.99 \AA\left(\mathrm{CH}_{2}\right)$ and with $U_{\text {iso }}(\mathrm{H})=$ 
Table 3

Experimental details.

(I) (II)

Crystal data

Chemical formula

$M_{\mathrm{r}}$

Crystal system, space group

Temperature (K)

$a, b, c(\AA)$

$\beta\left({ }^{\circ}\right)$

$V\left(\AA^{3}\right)$

$Z$

Radiation type

$\mu\left(\mathrm{mm}^{-1}\right)$

Crystal size (mm)

Data collection

Diffractometer

Absorption correction

$T_{\min }, T_{\max }$

No. of measured, independent and observed $[I>2 \sigma(I)]$ reflections

$R_{\text {int }}$

$(\sin \theta / \lambda)_{\max }\left(\AA^{-1}\right)$

$\mathrm{C}_{18} \mathrm{H}_{20} \mathrm{ClN}_{4}{ }^{+} \cdot \mathrm{C}_{7} \mathrm{H}_{3} \mathrm{~N}_{2} \mathrm{O}_{6}{ }^{-} \cdot \mathrm{C}_{2} \mathrm{H}_{6} \mathrm{OS}$

617.07

Monoclinic, $P 2_{1} / c$

173

15.3593 (2), 15.7685 (2),

$11.8679(2)$

$91.8097(14)$

$2872.89(7)$

4

$\mathrm{Cu} K \alpha$

2.34

$0.26 \times 0.22 \times 0.18$

(III)

Refinement

$R\left[F^{2}>2 \sigma\left(F^{2}\right)\right], w R\left(F^{2}\right), S$

No. of reflections

No. of parameters

No. of restraints

$\mathrm{H}$-atom treatment

Agilent Eos Gemini

Multi-scan (CrysAlis RED;

Agilent, 2012)

$0.424,0.656$

$19784,5527,4623$

0.032

0.614

$0.056,0.152,1.04$

5527

409

6

$\mathrm{H}$ atoms treated by a mixture of independent and constrained refinement

$0.89,-0.97$

$-$

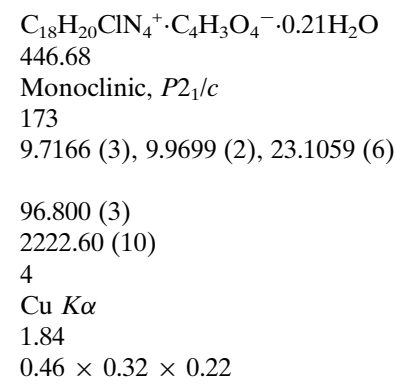

\begin{tabular}{|c|c|}
\hline Agilent Eos Gemini & Agilent Eos Gemini \\
\hline $\begin{array}{l}\text { Multi-scan (CrysAlis RED; } \\
\quad \text { Agilent, 2012) }\end{array}$ & $\begin{array}{l}\text { Multi-scan (CrysAlis RED; } \\
\quad \text { Agilent, 2012) }\end{array}$ \\
\hline $0.440,0.668$ & $0.399,0.705$ \\
\hline $8634,4228,3552$ & $7244,4069,3962$ \\
\hline 0.026 & 0.048 \\
\hline 0.614 & 0.619 \\
\hline $0.048,0.133,1.03$ & $0.056,0.141,1.08$ \\
\hline 4228 & 4069 \\
\hline 295 & 308 \\
\hline 0 & 2 \\
\hline $\begin{array}{l}\mathrm{H} \text { atoms treated by a mixture of } \\
\text { independent and constrained } \\
\text { refinement }\end{array}$ & $\begin{array}{l}\mathrm{H} \text { atoms treated by a mixture of } \\
\text { independent and constrained } \\
\text { refinement }\end{array}$ \\
\hline $0.46,-0.30$ & $0.53,-0.36$ \\
\hline- & $\begin{array}{l}\text { Flack } x \text { determined using } 1674 \\
\quad \text { quotients }\left[\left(I^{+}\right)-\left(I^{-}\right)\right] /\left[\left(I^{+}\right)+\left(I^{-}\right)\right] \\
\quad \text { (Parsons } \text { et al., } 2013)\end{array}$ \\
\hline- & $-0.022(17)$ \\
\hline
\end{tabular}

$k U_{\text {eq }}(\mathrm{C})$, where $k=1.5$ for the methyl groups, which were permitted to rotate but not to tilt, and 1.2 for all other $\mathrm{H}$ atoms bonded to $\mathrm{C}$ atoms. For the $\mathrm{H}$ atoms bonded to $\mathrm{N}$ or $\mathrm{O}$ atoms, the atomic coordinates were refined with $U_{\text {iso }}(\mathrm{H})=1.2 U_{\text {eq }}(\mathrm{N})$ or $1.5 U_{\mathrm{eq}}(\mathrm{O})$, giving the $\mathrm{N}-\mathrm{H}$ and $\mathrm{O}-\mathrm{H}$ distances shown in Table 1.

In compound (I), the dimethyl sulfoxide component is disordered over two sets of atomic sites having unequal occupancy (cf. Fig. 1). For the minor disorder component, the bonded distances and the one-angle non-bonded distances were all restrained to be the same as the corresponding distances in the major component subject to uncertainties of $0.005 \AA$ and $0.01 \AA$ respectively. The anisotropic displacement parameters for those pairs of partial-occupancy $\mathrm{C}$ and $\mathrm{O}$ atoms occupying essentially the same physical space were constrained to be identical, and the $\mathrm{H}$ atoms of the dimethyl sulfoxide components were included as riding atoms with $\mathrm{C}-$ $\mathrm{H}$ distances $0.95 \AA$ and $U_{\text {iso }}(\mathrm{H})=1.5 U_{\text {eq }}(\mathrm{C})$. Subject to these conditions, independent refinement of the site occupancies for the two disorder components gave values of 0.613 (3) and 0.359 (3): thereafter the occupancies were constrained to sum to unity, giving final values of 0.627 (2) and 0.373 (2). At this stage of the refinements there were no significant features in the difference maps for compounds (I) and (III), but for (II) there was a single significant peak, $1.51 \mathrm{e} \AA^{-3}$, which was within plausible hydrogen-bonding distance of two $\mathrm{O}$ atoms. Examination of the structures of compounds (I) and (III) using PLATON (Spek, 2009) showed that there were no solvent-accessible voids in these structures. However, in compound (II), there was a total void volume of $c a 88 \AA^{3}$ per unit cell, and examination of the structure of (II) using the SQUEEZE tool (Spek, 2015) within PLATON disclosed the presence of an addition 8.8 electrons per unit cell, equivalent to 0.22 molecules of water per ion pair. Accordingly, the large residual was modeled as the $\mathrm{O}$ atom, denoted $\mathrm{O} 31$, of a partial occupancy water molecule, which was refined isotropically: it was not possible to locate the $\mathrm{H}$ atoms associated with atom O31 in difference maps, but they were included in calculated positions with $\mathrm{O}-\mathrm{H} 0.90 \AA$ and $U_{\text {iso }}(\mathrm{H})=1.5 U_{\text {iso }}(\mathrm{O})$. Subject to these conditions, the occupancy of the water molecule refined to a value of 0.210 (7), very close to that indicated by SQUEEZE. It should be emphasized here that the application of the SQUEEZE procedure referred to above was intended only to estimate the number of electrons not yet accounted for 
at that stage of the refinement, and that the refinements at every stage were undertaken with the original data, independent of SQUEEZE. For compound (III), the correct orientation of the structure with respect to the polar axis directions was established by means of the Flack $x$ parameter (Flack, 1983), $x=-0.022$ (17), calculated (Parsons et al., 2013) using 1674 quotients of the type $\left[\left(I^{+}\right)-\left(I^{-}\right)\right] /\left[\left(I^{+}\right)+\left(I^{-}\right)\right]$.

\section{Acknowledgements}

MK thanks the UOM for research facilities and is also grateful to CPEPA, UGC, for the award of a JRF. JPJ acknowledges the NSF-MRI program (grant No. 1039027) for funds to purchase the X-ray diffractometer.

\section{References}

Agilent (2012). CrysAlis PRO and CrysAlis RED. Agilent Technologies Ltd, Yarnton, England.

Bernstein, J., Davis, R. E., Shimoni, L. \& Chang, N.-L. (1995). Angew. Chem. Int. Ed. Engl. 34, 1555-1573.
Breier, A., Buchanan, R. W., Kirkpatrick, B., David, O. R., Irish, D., Summerfelt, A. \& Carpenter, J. W. Jr (1994). Am. J. Psychiatry, 151, 20-26.

Ferguson, G., Glidewell, C., Gregson, R. M. \& Meehan, P. R. (1998a). Acta Cryst. B54, 129-138.

Ferguson, G., Glidewell, C., Gregson, R. M. \& Meehan, P. R. (1998b). Acta Cryst. B54, 139-150.

Fillers, J. P. \& Hawkinson, S. W. (1982). Acta Cryst. B38, 1750-1753.

Flack, H. D. (1983). Acta Cryst. A39, 876-881.

Gilli, P., Bertolasi, V., Ferretti, V. \& Gilli, G. (1994). J. Am. Chem. Soc. 116, 909-915.

Gregson, R. M., Glidewell, C., Ferguson, G. \& Lough, A. J. (2000). Acta Cryst. B56, 39-57.

Parsons, S., Flack, H. D. \& Wagner, T. (2013). Acta Cryst. B69, 249259.

Petcher, T. J. \& Weber, H.-P. (1976). J. Chem. Soc. Perkin Trans. 2, pp. 1415-1420.

Sheldrick, G. M. (2008). Acta Cryst. A64, 112-122.

Sheldrick, G. M. (2015). Acta Cryst. C71, 3-8.

Spek, A. L. (2009). Acta Cryst. D65, 148-155.

Spek, A. L. (2015). Acta Cryst. C71, 9-18.

Wood, P. A., Allen, F. H. \& Pidcock, E. (2009). CrystEngComm, 11, 1563-1571. 


\section{supporting information}

Acta Cryst. (2015). E71, 406-413 [doi:10.1107/S205698901500554X]

\section{The crystal structures of three clozapinium salts: different molecular} configurations, and supramolecular assembly in one, two and three dimensions

Manpreet Kaur, Jerry P. Jasinski, Hemmige S. Yathirajan, Channappa N. Kavitha and Christopher Glidewell

Computing details

For all compounds, data collection: CrysAlis PRO (Agilent, 2012); cell refinement: CrysAlis PRO (Agilent, 2012); data reduction: CrysAlis RED (Agilent, 2012); program(s) used to solve structure: SHELXS97 (Sheldrick, 2008); program(s) used to refine structure: SHELXL2014 (Sheldrick, 2015); molecular graphics: PLATON (Spek, 2009); software used to prepare material for publication: SHELXL2014 (Sheldrick, 2015) and PLATON (Spek, 2009).

(I) Clozapinium 3,5-dinitrobenzoate dimethyl sulfoxide monosolvate

Crystal data

$\mathrm{C}_{18} \mathrm{H}_{20} \mathrm{ClN}_{4}^{+} \cdot \mathrm{C}_{7} \mathrm{H}_{3} \mathrm{~N}_{2} \mathrm{O}_{6} \cdot \mathrm{C}_{2} \mathrm{H}_{6} \mathrm{OS}$

$M_{r}=617.07$

Monoclinic, $P 2_{1} / c$

$a=15.3593(2) \AA$

$b=15.7685(2) \AA$

$c=11.8679(2) \AA$

$\beta=91.8097(14)^{\circ}$

$V=2872.89(7) \AA^{3}$

$Z=4$

\section{Data collection}

Agilent Eos Gemini diffractometer

Radiation source: Enhance $(\mathrm{Cu}) \mathrm{X}$-ray Source $\omega$ scans

Absorption correction: multi-scan

(CrysAlis RED; Agilent, 2012)

$T_{\min }=0.424, T_{\max }=0.656$

19784 measured reflections

\section{Refinement}

Refinement on $F^{2}$

Least-squares matrix: full

$R\left[F^{2}>2 \sigma\left(F^{2}\right)\right]=0.056$

$w R\left(F^{2}\right)=0.152$

$S=1.04$

5527 reflections

409 parameters

6 restraints
$F(000)=1288$

$D_{\mathrm{x}}=1.427 \mathrm{Mg} \mathrm{m}^{-3}$

$\mathrm{Cu} K \alpha$ radiation, $\lambda=1.54184 \AA$

Cell parameters from 5527 reflections

$\theta=4.0-71.3^{\circ}$

$\mu=2.34 \mathrm{~mm}^{-1}$

$T=173 \mathrm{~K}$

Block, colourless

$0.26 \times 0.22 \times 0.18 \mathrm{~mm}$

5527 independent reflections

4623 reflections with $I>2 \sigma(I)$

$R_{\text {int }}=0.032$

$\theta_{\max }=71.3^{\circ}, \theta_{\min }=4.0^{\circ}$

$h=-17 \rightarrow 18$

$k=-19 \rightarrow 15$

$l=-14 \rightarrow 14$

Hydrogen site location: difference Fourier map

$\mathrm{H}$ atoms treated by a mixture of independent and constrained refinement

$w=1 /\left[\sigma^{2}\left(F_{\mathrm{o}}^{2}\right)+(0.0639 P)^{2}+2.7986 P\right]$

where $P=\left(F_{\mathrm{o}}{ }^{2}+2 F_{\mathrm{c}}{ }^{2}\right) / 3$

$(\Delta / \sigma)_{\max }<0.001$

$\Delta \rho_{\max }=0.89 \mathrm{e} \AA^{-3}$

$\Delta \rho_{\min }=-0.97$ e $\AA^{-3}$ 


\section{Special details}

Geometry. All e.s.d.'s (except the e.s.d. in the dihedral angle between two 1.s. planes) are estimated using the full covariance matrix. The cell e.s.d.'s are taken into account individually in the estimation of e.s.d.'s in distances, angles and torsion angles; correlations between e.s.d.'s in cell parameters are only used when they are defined by crystal symmetry. An approximate (isotropic) treatment of cell e.s.d.'s is used for estimating e.s.d.'s involving l.s. planes.

Fractional atomic coordinates and isotropic or equivalent isotropic displacement parameters $\left(\AA^{2}\right)$

\begin{tabular}{|c|c|c|c|c|c|}
\hline & $x$ & $y$ & $z$ & $U_{\text {iso }} * / U_{\text {eq }}$ & Occ. $(<1)$ \\
\hline $\mathrm{C} 1$ & $0.35763(15)$ & $0.19065(15)$ & $0.64680(19)$ & $0.0349(5)$ & \\
\hline $\mathrm{H} 1$ & 0.3526 & 0.1546 & 0.5828 & $0.042 *$ & \\
\hline $\mathrm{C} 2$ & $0.40839(15)$ & $0.16561(16)$ & $0.7394(2)$ & $0.0396(5)$ & \\
\hline $\mathrm{H} 2$ & 0.4388 & 0.1132 & 0.7385 & $0.048 *$ & \\
\hline $\mathrm{C} 3$ & $0.41440(16)$ & $0.21776(18)$ & $0.8335(2)$ & $0.0428(6)$ & \\
\hline H3 & 0.4452 & 0.1990 & 0.8996 & $0.051 *$ & \\
\hline $\mathrm{C} 4$ & $0.37586(16)$ & $0.29647(17)$ & $0.8315(2)$ & $0.0406(5)$ & \\
\hline $\mathrm{H} 4$ & 0.3830 & 0.3332 & 0.8945 & $0.049 *$ & \\
\hline $\mathrm{C} 4 \mathrm{~A}$ & $0.32657(14)$ & $0.32265(15)$ & $0.7379(2)$ & $0.0344(5)$ & \\
\hline N5 & $0.28834(14)$ & $0.40442(13)$ & $0.73413(19)$ & $0.0398(5)$ & \\
\hline H5 & $0.296(2)$ & $0.4270(19)$ & $0.797(3)$ & $0.048^{*}$ & \\
\hline $\mathrm{C} 5 \mathrm{~A}$ & $0.32343(14)$ & $0.45611(15)$ & $0.6491(2)$ & $0.0374(5)$ & \\
\hline C6 & $0.36736(17)$ & $0.53078(17)$ & $0.6785(3)$ & $0.0479(6)$ & \\
\hline H6 & 0.3744 & 0.5458 & 0.7557 & $0.057^{*}$ & \\
\hline $\mathrm{C} 7$ & $0.40067(17)$ & $0.58291(18)$ & $0.5976(3)$ & $0.0534(7)$ & \\
\hline $\mathrm{H} 7$ & 0.4290 & 0.6345 & 0.6180 & $0.064^{*}$ & \\
\hline $\mathrm{C} 8$ & $0.39217(16)$ & $0.55887(17)$ & $0.4864(3)$ & $0.0491(7)$ & \\
\hline $\mathrm{Cl} 8$ & $0.43801(6)$ & $0.62363(5)$ & $0.38475(8)$ & $0.0715(3)$ & \\
\hline C9 & $0.34849(16)$ & $0.48559(16)$ & $0.4543(2)$ & $0.0422(6)$ & \\
\hline H9 & 0.3430 & 0.4709 & 0.3767 & $0.051^{*}$ & \\
\hline C9A & $0.31242(14)$ & $0.43313(14)$ & $0.5354(2)$ & $0.0355(5)$ & \\
\hline N10 & $0.25884(12)$ & $0.36645(12)$ & $0.49775(17)$ & $0.0361(4)$ & \\
\hline C11 & $0.25516(14)$ & $0.29455(14)$ & $0.54945(19)$ & $0.0333(5)$ & \\
\hline C11A & $0.31376(14)$ & $0.26801(14)$ & $0.64620(19)$ & $0.0328(5)$ & \\
\hline N11 & $0.19690(14)$ & $0.23520(13)$ & $0.50890(17)$ & $0.0393(5)$ & \\
\hline $\mathrm{C} 12$ & $0.14714(16)$ & $0.24954(16)$ & $0.4036(2)$ & $0.0399(5)$ & \\
\hline $\mathrm{H} 12 \mathrm{~A}$ & 0.1621 & 0.2052 & 0.3484 & $0.048^{*}$ & \\
\hline H12B & 0.1634 & 0.3052 & 0.3720 & $0.048^{*}$ & \\
\hline $\mathrm{C} 13$ & $0.05023(16)$ & $0.24786(15)$ & $0.4213(2)$ & $0.0396(5)$ & \\
\hline $\mathrm{H} 13 \mathrm{~A}$ & 0.0340 & 0.2952 & 0.4712 & $0.047 *$ & \\
\hline H13B & 0.0184 & 0.2551 & 0.3480 & $0.047 *$ & \\
\hline N14 & $0.02552(12)$ & $0.16556(12)$ & $0.47312(16)$ & $0.0344(4)$ & \\
\hline H14 & $0.0420(18)$ & $0.1196(18)$ & $0.420(2)$ & $0.041 *$ & \\
\hline $\mathrm{C} 15$ & $0.07363(16)$ & $0.15561(16)$ & $0.5833(2)$ & $0.0387(5)$ & \\
\hline $\mathrm{H} 15 \mathrm{~A}$ & 0.0576 & 0.1010 & 0.6181 & $0.046^{*}$ & \\
\hline H15B & 0.0570 & 0.2018 & 0.6348 & $0.046^{*}$ & \\
\hline $\mathrm{C} 16$ & 0.17085 (16) & $0.15805(15)$ & $0.5671(2)$ & $0.0375(5)$ & \\
\hline H16A & 0.2016 & 0.1551 & 0.6415 & $0.045^{*}$ & \\
\hline H16B & 0.1882 & 0.1080 & 0.5227 & $0.045^{*}$ & \\
\hline
\end{tabular}




\begin{tabular}{|c|c|c|c|c|c|}
\hline $\mathrm{C} 17$ & $-0.06991(17)$ & $0.1565(2)$ & $0.4843(3)$ & $0.0531(7)$ & \\
\hline H17A & -0.0826 & 0.1017 & 0.5190 & $0.080^{*}$ & \\
\hline H17B & -0.0987 & 0.1595 & 0.4095 & $0.080 *$ & \\
\hline $\mathrm{H} 17 \mathrm{C}$ & -0.0915 & 0.2024 & 0.5316 & $0.080 *$ & \\
\hline $\mathrm{C} 21$ & $0.10211(14)$ & $-0.00223(14)$ & $0.1606(2)$ & $0.0328(5)$ & \\
\hline $\mathrm{C} 22$ & $0.14232(15)$ & $-0.07486(15)$ & $0.2025(2)$ & $0.0392(5)$ & \\
\hline $\mathrm{H} 22$ & 0.1445 & -0.0862 & 0.2811 & $0.047 *$ & \\
\hline $\mathrm{C} 23$ & $0.17913(16)$ & $-0.13049(15)$ & $0.1277(3)$ & $0.0457(6)$ & \\
\hline $\mathrm{C} 24$ & $0.17770(16)$ & $-0.11803(18)$ & $0.0129(3)$ & $0.0483(7)$ & \\
\hline $\mathrm{H} 24$ & 0.2032 & -0.1573 & -0.0370 & $0.058^{*}$ & \\
\hline $\mathrm{C} 25$ & $0.13710(15)$ & $-0.04533(18)$ & $-0.0255(2)$ & $0.0431(6)$ & \\
\hline $\mathrm{C} 26$ & 0.09975 (14) & $0.01300(15)$ & $0.0456(2)$ & $0.0362(5)$ & \\
\hline $\mathrm{H} 26$ & 0.0729 & 0.0628 & 0.0158 & $0.043^{*}$ & \\
\hline $\mathrm{C} 27$ & $0.06407(15)$ & $0.06224(15)$ & $0.2402(2)$ & $0.0359(5)$ & \\
\hline $\mathrm{O} 21$ & $0.03166(12)$ & $0.12736(11)$ & $0.19957(16)$ & $0.0466(4)$ & \\
\hline $\mathrm{O} 22$ & 0.07009 (12) & $0.04261(11)$ & $0.34434(15)$ & $0.0442(4)$ & \\
\hline $\mathrm{N} 23$ & $0.22603(19)$ & $-0.20507(16)$ & $0.1753(3)$ & $0.0687(8)$ & \\
\hline $\mathrm{O} 23$ & $0.2293(3)$ & $-0.21397(19)$ & $0.2776(3)$ & $0.1090(11)$ & \\
\hline $\mathrm{O} 24$ & $0.26036(17)$ & $-0.25228(15)$ & $0.1091(3)$ & $0.0895(9)$ & \\
\hline N25 & $0.13695(16)$ & $-0.0268(2)$ & $-0.1471(2)$ & $0.0619(7)$ & \\
\hline $\mathrm{O} 25$ & $0.17166(18)$ & $-0.0786(2)$ & $-0.2086(2)$ & $0.1021(11)$ & \\
\hline $\mathrm{O} 26$ & $0.10244(16)$ & $0.03914(18)$ & $-0.18044(18)$ & $0.0696(7)$ & \\
\hline S31 & $0.31808(7)$ & $0.56967(6)$ & $1.00103(8)$ & $0.0448(3)$ & $0.627(2)$ \\
\hline $\mathrm{O} 31$ & $0.3389(3)$ & $0.4857(2)$ & $0.9475(3)$ & $0.0655(11)$ & $0.627(2)$ \\
\hline $\mathrm{C} 31$ & $0.4020(7)$ & $0.6409(4)$ & $0.9689(7)$ & $0.083(3)$ & $0.627(2)$ \\
\hline $\mathrm{H} 31 \mathrm{~A}$ & 0.3963 & 0.6574 & 0.8894 & $0.125^{*}$ & $0.627(2)$ \\
\hline H31B & 0.4586 & 0.6135 & 0.9830 & $0.125^{*}$ & $0.627(2)$ \\
\hline $\mathrm{H} 31 \mathrm{C}$ & 0.3979 & 0.6914 & 1.0165 & $0.125^{*}$ & $0.627(2)$ \\
\hline C32 & $0.3429(6)$ & $0.5547(4)$ & $1.1451(4)$ & $0.081(2)$ & $0.627(2)$ \\
\hline $\mathrm{H} 32 \mathrm{~A}$ & 0.4050 & 0.5416 & 1.1557 & $0.122 *$ & $0.627(2)$ \\
\hline H32B & 0.3082 & 0.5076 & 1.1735 & $0.122 *$ & $0.627(2)$ \\
\hline $\mathrm{H} 32 \mathrm{C}$ & 0.3293 & 0.6066 & 1.1865 & $0.122 *$ & $0.627(2)$ \\
\hline S41 & $0.39889(18)$ & $0.53363(13)$ & $1.05795(17)$ & $0.0678(8)$ & $0.373(2)$ \\
\hline O41 & $0.3879(5)$ & $0.4760(4)$ & $0.9582(6)$ & $0.0655(11)$ & $0.373(2)$ \\
\hline $\mathrm{C} 41$ & $0.4247(12)$ & $0.6356(6)$ & $1.0098(12)$ & $0.083(3)$ & $0.373(2)$ \\
\hline $\mathrm{H} 41 \mathrm{~A}$ & 0.3746 & 0.6590 & 0.9670 & $0.125^{*}$ & $0.373(2)$ \\
\hline H41B & 0.4749 & 0.6323 & 0.9610 & $0.125^{*}$ & $0.373(2)$ \\
\hline $\mathrm{H} 41 \mathrm{C}$ & 0.4388 & 0.6723 & 1.0744 & $0.125^{*}$ & $0.373(2)$ \\
\hline $\mathrm{C} 42$ & $0.2944(5)$ & $0.5526(6)$ & $1.1067(8)$ & $0.081(2)$ & $0.373(2)$ \\
\hline $\mathrm{H} 42 \mathrm{~A}$ & 0.2725 & 0.5012 & 1.1426 & $0.122^{*}$ & $0.373(2)$ \\
\hline $\mathrm{H} 42 \mathrm{~B}$ & 0.2554 & 0.5683 & 1.0432 & $0.122 *$ & $0.373(2)$ \\
\hline $\mathrm{H} 42 \mathrm{C}$ & 0.2967 & 0.5989 & 1.1618 & $0.122 *$ & $0.373(2)$ \\
\hline
\end{tabular}

Atomic displacement parameters $\left(\AA^{2}\right)$

\begin{tabular}{lllllll}
\hline & $U^{11}$ & $U^{22}$ & $U^{33}$ & $U^{12}$ & $U^{13}$ & $U^{23}$ \\
\hline $\mathrm{C} 1$ & $0.0350(11)$ & $0.0347(11)$ & $0.0348(11)$ & $0.0028(9)$ & $-0.0013(9)$ & $-0.0032(9)$ \\
$\mathrm{C} 2$ & $0.0350(12)$ & $0.0400(13)$ & $0.0437(13)$ & $0.0097(10)$ & $-0.0005(10)$ & $0.0000(10)$
\end{tabular}




\begin{tabular}{|c|c|c|c|c|c|c|}
\hline $\mathrm{C} 3$ & $0.0363(12)$ & $0.0554(15)$ & $0.0363(12)$ & $0.0065(11)$ & $-0.0053(10)$ & $0.0002(11)$ \\
\hline $\mathrm{C} 4$ & $0.0372(12)$ & $0.0489(14)$ & $0.0357(12)$ & $0.0012(11)$ & $-0.0011(10)$ & $-0.0089(10)$ \\
\hline $\mathrm{C} 4 \mathrm{~A}$ & $0.0283(11)$ & $0.0352(12)$ & $0.0398(12)$ & $0.0012(9)$ & $0.0030(9)$ & $-0.0039(9)$ \\
\hline N5 & $0.0391(11)$ & $0.0353(11)$ & $0.0452(12)$ & $0.0046(9)$ & $0.0042(9)$ & $-0.0088(9)$ \\
\hline $\mathrm{C} 5 \mathrm{~A}$ & $0.0257(10)$ & $0.0322(11)$ & $0.0543(14)$ & $0.0039(9)$ & $0.0006(10)$ & $-0.0038(10)$ \\
\hline C6 & $0.0348(13)$ & $0.0416(14)$ & $0.0670(17)$ & $-0.0027(11)$ & $-0.0037(12)$ & $-0.0089(12)$ \\
\hline $\mathrm{C} 7$ & $0.0365(13)$ & $0.0398(14)$ & $0.084(2)$ & $-0.0080(11)$ & -0.0040 & $-0.0051(14)$ \\
\hline $\mathrm{C} 8$ & $0.0301(12)$ & $0.0383(13)$ & $0.079(2)$ & $-0.0014(10)$ & $0.0071(12)$ & $0.0105(13)$ \\
\hline $\mathrm{Cl} 8$ & $0.0632(5)$ & $0.0533(4)$ & $0.0991(6)$ & $-0.0144(4)$ & $0.0176(4)$ & $0.0167(4)$ \\
\hline C9 & $0.0332(12)$ & $0.0361(12)$ & $0.0573(15)$ & $0.0052(10)$ & $0.0037(11)$ & $0.0031(11)$ \\
\hline C9A & $0.0237(10)$ & $0.0291(11)$ & $0.0534(14)$ & $0.0042(9)$ & $-0.0015(9)$ & $0.0004(10)$ \\
\hline N10 & $0.0305(9)$ & $0.0315(10)$ & $0.0459(11)$ & $0.0015(8)$ & $-0.0031(8)$ & $0.0014(8)$ \\
\hline $\mathrm{C} 11$ & $0.0293(11)$ & $0.0331(11)$ & $0.0373(12)$ & $0.0029(9)$ & $-0.0015(9)$ & $-0.0012(9)$ \\
\hline C11A & $0.0288(10)$ & $0.0328(11)$ & $0.0365(11)$ & $0.0005(9)$ & $-0.0016(9)$ & $-0.0009(9)$ \\
\hline N11 & $0.0423(11)$ & $0.0353(10)$ & $0.0394(10)$ & $-0.0076(9)$ & $-0.0112(9)$ & $0.0057(8)$ \\
\hline $\mathrm{C} 12$ & $0.0437(13)$ & $0.0390(13)$ & $0.0362(12)$ & $-0.0066(10)$ & $-0.0098(10)$ & $0.0055(10)$ \\
\hline $\mathrm{C} 13$ & $0.0435(13)$ & $0.0321(12)$ & $0.0426(13)$ & $0.0041(10)$ & $-0.0073(10)$ & $0.0016(10)$ \\
\hline N14 & $0.0316(10)$ & $0.0342(10)$ & $0.0373(10)$ & $0.0035(8)$ & $-0.0008(8)$ & $-0.0004(8)$ \\
\hline $\mathrm{C} 15$ & $0.0434(13)$ & $0.0372(12)$ & $0.0354(12)$ & $0.0004(10)$ & $-0.0015(10)$ & $0.0017(10)$ \\
\hline $\mathrm{C} 16$ & $0.0402(12)$ & $0.0327(12)$ & $0.0389(12)$ & $-0.0025(10)$ & $-0.0094(10)$ & $0.0053(9)$ \\
\hline $\mathrm{C} 17$ & $0.0336(13)$ & $0.0621(18)$ & $0.0636(18)$ & $0.0058(12)$ & $0.0029(12)$ & $0.0058(14)$ \\
\hline $\mathrm{C} 21$ & $0.0254(10)$ & $0.0292(11)$ & $0.0436(12)$ & $-0.0045(9)$ & $-0.0015(9)$ & $-0.0033(9)$ \\
\hline $\mathrm{C} 22$ & $0.0352(12)$ & $0.0313(11)$ & $0.0510(14)$ & $-0.0026(10)$ & $-0.0029(10)$ & $-0.0001(10)$ \\
\hline $\mathrm{C} 23$ & $0.0332(12)$ & $0.0296(12)$ & $0.0742(19)$ & $-0.0001(10)$ & $-0.0026(12)$ & $-0.0076(12)$ \\
\hline $\mathrm{C} 24$ & $0.0305(12)$ & $0.0480(15)$ & $0.0665(18)$ & $-0.0061(11)$ & $0.0048(11)$ & $-0.0243(13)$ \\
\hline $\mathrm{C} 25$ & $0.0290(11)$ & $0.0542(15)$ & $0.0459(14)$ & $-0.0113(11)$ & $0.0010(10)$ & $-0.0122(11)$ \\
\hline $\mathrm{C} 26$ & $0.0262(11)$ & $0.0364(12)$ & $0.0458(13)$ & $-0.0076(9)$ & $-0.0025(9)$ & $-0.0015(10)$ \\
\hline $\mathrm{C} 27$ & $0.0308(11)$ & $0.0323(12)$ & $0.0445(13)$ & $-0.0032(9)$ & $-0.0008(9)$ & $-0.0059(10)$ \\
\hline $\mathrm{O} 21$ & $0.0480(10)$ & $0.0359(9)$ & $0.0554(11)$ & $0.0104(8)$ & $-0.0054(8)$ & $-0.0052(8)$ \\
\hline $\mathrm{O} 22$ & $0.0519(10)$ & $0.0388(9)$ & $0.0420(9)$ & $0.0027(8)$ & $0.0013(8)$ & $-0.0051(7)$ \\
\hline N23 & $0.0586(16)$ & $0.0345(13)$ & $0.113(3)$ & $0.0082(12)$ & $-0.0038(16)$ & $-0.0085(15)$ \\
\hline $\mathrm{O} 23$ & $0.142(3)$ & $0.0668(18)$ & $0.117(3)$ & $0.0490(19)$ & $-0.017(2)$ & $0.0159(17)$ \\
\hline $\mathrm{O} 24$ & $0.0714(16)$ & $0.0442(12)$ & $0.153(3)$ & $0.0188(12)$ & $0.0088(16)$ & $-0.0227(15)$ \\
\hline N25 & $0.0389(12)$ & $0.100(2)$ & $0.0472(13)$ & $-0.0174(14)$ & $0.0037(10)$ & $-0.0139(15)$ \\
\hline $\mathrm{O} 25$ & $0.0687(16)$ & $0.179(3)$ & $0.0587(15)$ & $0.0199(19)$ & $0.0102(12)$ & $-0.0409(18)$ \\
\hline $\mathrm{O} 26$ & $0.0705(15)$ & $0.0915(18)$ & $0.0462(12)$ & $-0.0312(14)$ & $-0.0086(10)$ & $0.0062(12)$ \\
\hline $\mathrm{S} 31$ & $0.0570(7)$ & $0.0362(5)$ & $0.0410(5)$ & $-0.0028(4)$ & $-0.0024(4)$ & $-0.0033(4)$ \\
\hline $\mathrm{O} 31$ & $0.094(3)$ & $0.0484(16)$ & $0.0543(16)$ & $-0.009(2)$ & $0.009(2)$ & $-0.0199(13)$ \\
\hline $\mathrm{C} 31$ & $0.081(6)$ & $0.060(2)$ & $0.111(7)$ & -0.030 & $0.040(6)$ & $-0.037(3)$ \\
\hline $\mathrm{C} 32$ & $0.145(7)$ & $0.054(2)$ & $0.044(3)$ & $0.021(4)$ & $-0.006(3)$ & $-0.002(2)$ \\
\hline S41 & $0.109(2)$ & $0.0467(11)$ & $0.0475(11)$ & $0.0257(11)$ & $0.0001(11)$ & $-0.0104(8)$ \\
\hline $\mathrm{O} 41$ & $0.094(3)$ & $0.0484(16)$ & $0.0543(16)$ & $-0.009(2)$ & $0.009(2)$ & $-0.0199(13)$ \\
\hline $\mathrm{C} 41$ & $0.081(6)$ & $0.060(2)$ & $0.111(7)$ & $-0.030(3)$ & $0.040(6)$ & $-0.037(3)$ \\
\hline $\mathrm{C} 42$ & $0.145(7)$ & $0.054(2)$ & 0.044 & $0.021(4)$ & $-0.006(3)$ & $-0.002(2)$ \\
\hline
\end{tabular}


Geometric parameters $\left(\AA,{ }^{\circ}\right)$

\begin{tabular}{|c|c|c|c|}
\hline $\mathrm{C} 1-\mathrm{C} 2$ & $1.385(3)$ & $\mathrm{C} 16-\mathrm{H} 16 \mathrm{~A}$ & 0.9900 \\
\hline $\mathrm{C} 1-\mathrm{C} 11 \mathrm{~A}$ & $1.394(3)$ & $\mathrm{C} 16-\mathrm{H} 16 \mathrm{~B}$ & 0.9900 \\
\hline $\mathrm{C} 1-\mathrm{H} 1$ & 0.9500 & $\mathrm{C} 17-\mathrm{H} 17 \mathrm{~A}$ & 0.9800 \\
\hline $\mathrm{C} 2-\mathrm{C} 3$ & $1.388(4)$ & C17-H17B & 0.9800 \\
\hline $\mathrm{C} 2-\mathrm{H} 2$ & 0.9500 & $\mathrm{C} 17-\mathrm{H} 17 \mathrm{C}$ & 0.9800 \\
\hline $\mathrm{C} 3-\mathrm{C} 4$ & $1.375(4)$ & $\mathrm{C} 21-\mathrm{C} 22$ & $1.386(3)$ \\
\hline $\mathrm{C} 3-\mathrm{H} 3$ & 0.9500 & $\mathrm{C} 21-\mathrm{C} 26$ & $1.386(3)$ \\
\hline $\mathrm{C} 4-\mathrm{C} 4 \mathrm{~A}$ & $1.387(3)$ & $\mathrm{C} 21-\mathrm{C} 27$ & $1.517(3)$ \\
\hline $\mathrm{C} 4-\mathrm{H} 4$ & 0.9500 & $\mathrm{C} 22-\mathrm{C} 23$ & $1.381(4)$ \\
\hline $\mathrm{C} 4 \mathrm{~A}-\mathrm{C} 11 \mathrm{~A}$ & $1.397(3)$ & $\mathrm{C} 22-\mathrm{H} 22$ & 0.9500 \\
\hline $\mathrm{C} 4 \mathrm{~A}-\mathrm{N} 5$ & $1.417(3)$ & $\mathrm{C} 23-\mathrm{C} 24$ & $1.376(4)$ \\
\hline $\mathrm{N} 5-\mathrm{C} 5 \mathrm{~A}$ & $1.417(3)$ & $\mathrm{C} 23-\mathrm{N} 23$ & $1.482(4)$ \\
\hline N5- H5 & $0.83(3)$ & $\mathrm{C} 24-\mathrm{C} 25$ & $1.376(4)$ \\
\hline $\mathrm{C} 5 \mathrm{~A}-\mathrm{C} 6$ & $1.396(3)$ & $\mathrm{C} 24-\mathrm{H} 24$ & 0.9500 \\
\hline $\mathrm{C} 5 \mathrm{~A}-\mathrm{C} 9 \mathrm{~A}$ & $1.402(4)$ & $\mathrm{C} 25-\mathrm{C} 26$ & $1.384(4)$ \\
\hline $\mathrm{C} 6-\mathrm{C} 7$ & $1.375(4)$ & $\mathrm{C} 25-\mathrm{N} 25$ & $1.473(4)$ \\
\hline C6-H6 & 0.9500 & $\mathrm{C} 26-\mathrm{H} 26$ & 0.9500 \\
\hline $\mathrm{C} 7-\mathrm{C} 8$ & $1.375(4)$ & $\mathrm{C} 27-\mathrm{O} 21$ & $1.233(3)$ \\
\hline $\mathrm{C} 7-\mathrm{H} 7$ & 0.9500 & $\mathrm{C} 27-\mathrm{O} 22$ & $1.274(3)$ \\
\hline $\mathrm{C} 8-\mathrm{C} 9$ & $1.384(4)$ & $\mathrm{N} 23-\mathrm{O} 24$ & $1.215(4)$ \\
\hline $\mathrm{C} 8-\mathrm{Cl} 8$ & $1.746(3)$ & $\mathrm{N} 23-\mathrm{O} 23$ & $1.222(4)$ \\
\hline $\mathrm{C} 9-\mathrm{C} 9 \mathrm{~A}$ & $1.397(4)$ & $\mathrm{N} 25-\mathrm{O} 26$ & $1.227(4)$ \\
\hline C9- $\mathrm{H} 9$ & 0.9500 & $\mathrm{~N} 25-\mathrm{O} 25$ & $1.228(4)$ \\
\hline $\mathrm{C} 9 \mathrm{~A}-\mathrm{N} 10$ & $1.400(3)$ & $\mathrm{S} 31-\mathrm{O} 31$ & $1.507(3)$ \\
\hline $\mathrm{N} 10-\mathrm{C} 11$ & $1.291(3)$ & $\mathrm{S} 31-\mathrm{C} 32$ & $1.756(5)$ \\
\hline $\mathrm{C} 11-\mathrm{N} 11$ & $1.372(3)$ & $\mathrm{S} 31-\mathrm{C} 31$ & $1.760(5)$ \\
\hline $\mathrm{C} 11-\mathrm{C} 11 \mathrm{~A}$ & $1.496(3)$ & $\mathrm{C} 31-\mathrm{H} 31 \mathrm{~A}$ & 0.9800 \\
\hline $\mathrm{N} 11-\mathrm{C} 16$ & $1.461(3)$ & $\mathrm{C} 31-\mathrm{H} 31 \mathrm{~B}$ & 0.9800 \\
\hline $\mathrm{N} 11-\mathrm{C} 12$ & $1.461(3)$ & $\mathrm{C} 31-\mathrm{H} 31 \mathrm{C}$ & 0.9800 \\
\hline $\mathrm{C} 12-\mathrm{C} 13$ & $1.510(4)$ & $\mathrm{C} 32-\mathrm{H} 32 \mathrm{~A}$ & 0.9800 \\
\hline $\mathrm{C} 12-\mathrm{H} 12 \mathrm{~A}$ & 0.9900 & $\mathrm{C} 32-\mathrm{H} 32 \mathrm{~B}$ & 0.9800 \\
\hline $\mathrm{C} 12-\mathrm{H} 12 \mathrm{~B}$ & 0.9900 & $\mathrm{C} 32-\mathrm{H} 32 \mathrm{C}$ & 0.9800 \\
\hline C13-N14 & $1.491(3)$ & $\mathrm{S} 41-\mathrm{O} 41$ & $1.497(4)$ \\
\hline C13-H13A & 0.9900 & $\mathrm{~S} 41-\mathrm{C} 42$ & $1.749(6)$ \\
\hline C13-H13B & 0.9900 & $\mathrm{~S} 41-\mathrm{C} 41$ & $1.756(6)$ \\
\hline $\mathrm{N} 14-\mathrm{C} 17$ & $1.483(3)$ & $\mathrm{C} 41-\mathrm{H} 41 \mathrm{~A}$ & 0.9800 \\
\hline $\mathrm{N} 14-\mathrm{C} 15$ & $1.490(3)$ & $\mathrm{C} 41-\mathrm{H} 41 \mathrm{~B}$ & 0.9800 \\
\hline N14-H14 & $1.00(3)$ & $\mathrm{C} 41-\mathrm{H} 41 \mathrm{C}$ & 0.9800 \\
\hline $\mathrm{C} 15-\mathrm{C} 16$ & $1.512(3)$ & $\mathrm{C} 42-\mathrm{H} 42 \mathrm{~A}$ & 0.9800 \\
\hline $\mathrm{C} 15-\mathrm{H} 15 \mathrm{~A}$ & 0.9900 & $\mathrm{C} 42-\mathrm{H} 42 \mathrm{~B}$ & 0.9800 \\
\hline C15-H15B & 0.9900 & $\mathrm{C} 42-\mathrm{H} 42 \mathrm{C}$ & 0.9800 \\
\hline $\mathrm{C} 2-\mathrm{C} 1-\mathrm{C} 11 \mathrm{~A}$ & $120.9(2)$ & $\mathrm{N} 11-\mathrm{C} 16-\mathrm{C} 15$ & $111.57(19)$ \\
\hline $\mathrm{C} 2-\mathrm{C} 1-\mathrm{H} 1$ & 119.5 & $\mathrm{~N} 11-\mathrm{C} 16-\mathrm{H} 16 \mathrm{~A}$ & 109.3 \\
\hline $\mathrm{C} 11 \mathrm{~A}-\mathrm{C} 1-\mathrm{H} 1$ & 119.5 & $\mathrm{C} 15-\mathrm{C} 16-\mathrm{H} 16 \mathrm{~A}$ & 109.3 \\
\hline $\mathrm{C} 1-\mathrm{C} 2-\mathrm{C} 3$ & $119.4(2)$ & $\mathrm{N} 11-\mathrm{C} 16-\mathrm{H} 16 \mathrm{~B}$ & 109.3 \\
\hline
\end{tabular}




\begin{tabular}{|c|c|}
\hline $\mathrm{C} 1-\mathrm{C} 2-\mathrm{H} 2$ & 120.3 \\
\hline $\mathrm{C} 3-\mathrm{C} 2-\mathrm{H} 2$ & 120.3 \\
\hline $\mathrm{C} 4-\mathrm{C} 3-\mathrm{C} 2$ & $120.2(2)$ \\
\hline $\mathrm{C} 4-\mathrm{C} 3-\mathrm{H} 3$ & 119.9 \\
\hline $\mathrm{C} 2-\mathrm{C} 3-\mathrm{H} 3$ & 119.9 \\
\hline $\mathrm{C} 3-\mathrm{C} 4-\mathrm{C} 4 \mathrm{~A}$ & $120.5(2)$ \\
\hline $\mathrm{C} 3-\mathrm{C} 4-\mathrm{H} 4$ & 119.8 \\
\hline $\mathrm{C} 4 \mathrm{~A}-\mathrm{C} 4-\mathrm{H} 4$ & 119.8 \\
\hline $\mathrm{C} 4-\mathrm{C} 4 \mathrm{~A}-\mathrm{C} 11 \mathrm{~A}$ & $120.0(2)$ \\
\hline $\mathrm{C} 4-\mathrm{C} 4 \mathrm{~A}-\mathrm{N} 5$ & $120.8(2)$ \\
\hline $\mathrm{C} 11 \mathrm{~A}-\mathrm{C} 4 \mathrm{~A}-\mathrm{N} 5$ & $119.2(2)$ \\
\hline $\mathrm{C} 4 \mathrm{~A}-\mathrm{N} 5-\mathrm{C} 5 \mathrm{~A}$ & $112.30(19)$ \\
\hline $\mathrm{C} 4 \mathrm{~A}-\mathrm{N} 5-\mathrm{H} 5$ & $109(2)$ \\
\hline $\mathrm{C} 5 \mathrm{~A}-\mathrm{N} 5-\mathrm{H} 5$ & $110(2)$ \\
\hline $\mathrm{C} 6-\mathrm{C} 5 \mathrm{~A}-\mathrm{C} 9 \mathrm{~A}$ & $120.1(2)$ \\
\hline $\mathrm{C} 6-\mathrm{C} 5 \mathrm{~A}-\mathrm{N} 5$ & $119.9(2)$ \\
\hline $\mathrm{C} 9 \mathrm{~A}-\mathrm{C} 5 \mathrm{~A}-\mathrm{N} 5$ & $120.0(2)$ \\
\hline $\mathrm{C} 7-\mathrm{C} 6-\mathrm{C} 5 \mathrm{~A}$ & $121.1(3)$ \\
\hline $\mathrm{C} 7-\mathrm{C} 6-\mathrm{H} 6$ & 119.4 \\
\hline $\mathrm{C} 5 \mathrm{~A}-\mathrm{C} 6-\mathrm{H} 6$ & 119.4 \\
\hline $\mathrm{C} 8-\mathrm{C} 7-\mathrm{C} 6$ & $118.6(3)$ \\
\hline $\mathrm{C} 8-\mathrm{C} 7-\mathrm{H} 7$ & 120.7 \\
\hline $\mathrm{C} 6-\mathrm{C} 7-\mathrm{H} 7$ & 120.7 \\
\hline $\mathrm{C} 7-\mathrm{C} 8-\mathrm{C} 9$ & $121.7(3)$ \\
\hline $\mathrm{C} 7-\mathrm{C} 8-\mathrm{Cl} 8$ & $118.3(2)$ \\
\hline $\mathrm{C} 9-\mathrm{C} 8-\mathrm{Cl} 8$ & $120.0(2)$ \\
\hline $\mathrm{C} 8-\mathrm{C} 9-\mathrm{C} 9 \mathrm{~A}$ & $120.3(3)$ \\
\hline $\mathrm{C} 8-\mathrm{C} 9-\mathrm{H} 9$ & 119.9 \\
\hline $\mathrm{C} 9 \mathrm{~A}-\mathrm{C} 9-\mathrm{H} 9$ & 119.9 \\
\hline $\mathrm{C} 9-\mathrm{C} 9 \mathrm{~A}-\mathrm{N} 10$ & $117.8(2)$ \\
\hline $\mathrm{C} 9-\mathrm{C} 9 \mathrm{~A}-\mathrm{C} 5 \mathrm{~A}$ & $118.1(2)$ \\
\hline $\mathrm{N} 10-\mathrm{C} 9 \mathrm{~A}-\mathrm{C} 5 \mathrm{~A}$ & $123.6(2)$ \\
\hline $\mathrm{C} 11-\mathrm{N} 10-\mathrm{C} 9 \mathrm{~A}$ & $122.8(2)$ \\
\hline $\mathrm{N} 10-\mathrm{C} 11-\mathrm{N} 11$ & $118.1(2)$ \\
\hline $\mathrm{N} 10-\mathrm{C} 11-\mathrm{C} 11 \mathrm{~A}$ & $125.2(2)$ \\
\hline $\mathrm{N} 11-\mathrm{C} 11-\mathrm{C} 11 \mathrm{~A}$ & $116.6(2)$ \\
\hline $\mathrm{C} 1-\mathrm{C} 11 \mathrm{~A}-\mathrm{C} 4 \mathrm{~A}$ & $118.7(2)$ \\
\hline $\mathrm{C} 1-\mathrm{C} 11 \mathrm{~A}-\mathrm{C} 11$ & $121.9(2)$ \\
\hline $\mathrm{C} 4 \mathrm{~A}-\mathrm{C} 11 \mathrm{~A}-\mathrm{C} 11$ & $119.5(2)$ \\
\hline $\mathrm{C} 11-\mathrm{N} 11-\mathrm{C} 16$ & $126.01(19)$ \\
\hline $\mathrm{C} 11-\mathrm{N} 11-\mathrm{C} 12$ & $120.80(19)$ \\
\hline $\mathrm{C} 16-\mathrm{N} 11-\mathrm{C} 12$ & $112.91(18)$ \\
\hline $\mathrm{N} 11-\mathrm{C} 12-\mathrm{C} 13$ & $111.7(2)$ \\
\hline $\mathrm{N} 11-\mathrm{C} 12-\mathrm{H} 12 \mathrm{~A}$ & 109.3 \\
\hline $\mathrm{C} 13-\mathrm{C} 12-\mathrm{H} 12 \mathrm{~A}$ & 109.3 \\
\hline $\mathrm{N} 11-\mathrm{C} 12-\mathrm{H} 12 \mathrm{~B}$ & 109.3 \\
\hline $\mathrm{C} 13-\mathrm{C} 12-\mathrm{H} 12 \mathrm{~B}$ & 109.3 \\
\hline & \\
\hline
\end{tabular}

\begin{tabular}{|c|c|}
\hline $\mathrm{C} 15-\mathrm{C} 16-\mathrm{H} 16 \mathrm{~B}$ & 109.3 \\
\hline $\mathrm{H} 16 \mathrm{~A}-\mathrm{C} 16-\mathrm{H} 16 \mathrm{~B}$ & 108.0 \\
\hline $\mathrm{N} 14-\mathrm{C} 17-\mathrm{H} 17 \mathrm{~A}$ & 109.5 \\
\hline $\mathrm{N} 14-\mathrm{C} 17-\mathrm{H} 17 \mathrm{~B}$ & 109.5 \\
\hline $\mathrm{H} 17 \mathrm{~A}-\mathrm{C} 17-\mathrm{H} 17 \mathrm{~B}$ & 109.5 \\
\hline $\mathrm{N} 14-\mathrm{C} 17-\mathrm{H} 17 \mathrm{C}$ & 109.5 \\
\hline $\mathrm{H} 17 \mathrm{~A}-\mathrm{C} 17-\mathrm{H} 17 \mathrm{C}$ & 109.5 \\
\hline $\mathrm{H} 17 \mathrm{~B}-\mathrm{C} 17-\mathrm{H} 17 \mathrm{C}$ & 109.5 \\
\hline $\mathrm{C} 22-\mathrm{C} 21-\mathrm{C} 26$ & $119.6(2)$ \\
\hline $\mathrm{C} 22-\mathrm{C} 21-\mathrm{C} 27$ & $120.5(2)$ \\
\hline $\mathrm{C} 26-\mathrm{C} 21-\mathrm{C} 27$ & $119.9(2)$ \\
\hline $\mathrm{C} 23-\mathrm{C} 22-\mathrm{C} 21$ & $118.8(2)$ \\
\hline $\mathrm{C} 23-\mathrm{C} 22-\mathrm{H} 22$ & 120.6 \\
\hline $\mathrm{C} 21-\mathrm{C} 22-\mathrm{H} 22$ & 120.6 \\
\hline $\mathrm{C} 24-\mathrm{C} 23-\mathrm{C} 22$ & $123.5(2)$ \\
\hline $\mathrm{C} 24-\mathrm{C} 23-\mathrm{N} 23$ & $118.9(3)$ \\
\hline $\mathrm{C} 22-\mathrm{C} 23-\mathrm{N} 23$ & $117.6(3)$ \\
\hline $\mathrm{C} 25-\mathrm{C} 24-\mathrm{C} 23$ & $116.1(2)$ \\
\hline $\mathrm{C} 25-\mathrm{C} 24-\mathrm{H} 24$ & 122.0 \\
\hline $\mathrm{C} 23-\mathrm{C} 24-\mathrm{H} 24$ & 122.0 \\
\hline $\mathrm{C} 24-\mathrm{C} 25-\mathrm{C} 26$ & $123.0(3)$ \\
\hline $\mathrm{C} 24-\mathrm{C} 25-\mathrm{N} 25$ & $118.4(3)$ \\
\hline $\mathrm{C} 26-\mathrm{C} 25-\mathrm{N} 25$ & $118.5(3)$ \\
\hline $\mathrm{C} 25-\mathrm{C} 26-\mathrm{C} 21$ & $119.1(2)$ \\
\hline $\mathrm{C} 25-\mathrm{C} 26-\mathrm{H} 26$ & 120.4 \\
\hline $\mathrm{C} 21-\mathrm{C} 26-\mathrm{H} 26$ & 120.4 \\
\hline $\mathrm{O} 21-\mathrm{C} 27-\mathrm{O} 22$ & $126.7(2)$ \\
\hline $\mathrm{O} 21-\mathrm{C} 27-\mathrm{C} 21$ & $118.2(2)$ \\
\hline $\mathrm{O} 22-\mathrm{C} 27-\mathrm{C} 21$ & $115.0(2)$ \\
\hline $\mathrm{O} 24-\mathrm{N} 23-\mathrm{O} 23$ & $124.6(3)$ \\
\hline $\mathrm{O} 24-\mathrm{N} 23-\mathrm{C} 23$ & $117.1(3)$ \\
\hline $\mathrm{O} 23-\mathrm{N} 23-\mathrm{C} 23$ & $118.3(3)$ \\
\hline $\mathrm{O} 26-\mathrm{N} 25-\mathrm{O} 25$ & $124.3(3)$ \\
\hline $\mathrm{O} 26-\mathrm{N} 25-\mathrm{C} 25$ & $118.2(3)$ \\
\hline $\mathrm{O} 25-\mathrm{N} 25-\mathrm{C} 25$ & $117.6(3)$ \\
\hline $\mathrm{O} 31-\mathrm{S} 31-\mathrm{C} 32$ & $104.5(2)$ \\
\hline $\mathrm{O} 31-\mathrm{S} 31-\mathrm{C} 31$ & $107.6(3)$ \\
\hline $\mathrm{C} 32-\mathrm{S} 31-\mathrm{C} 31$ & $99.1(4)$ \\
\hline $\mathrm{S} 31-\mathrm{C} 31-\mathrm{H} 31 \mathrm{~A}$ & 109.5 \\
\hline $\mathrm{S} 31-\mathrm{C} 31-\mathrm{H} 31 \mathrm{~B}$ & 109.5 \\
\hline $\mathrm{H} 31 \mathrm{~A}-\mathrm{C} 31-\mathrm{H} 31 \mathrm{~B}$ & 109.5 \\
\hline $\mathrm{S} 31-\mathrm{C} 31-\mathrm{H} 31 \mathrm{C}$ & 109.5 \\
\hline $\mathrm{H} 31 \mathrm{~A}-\mathrm{C} 31-\mathrm{H} 31 \mathrm{C}$ & 109.5 \\
\hline $\mathrm{H} 31 \mathrm{~B}-\mathrm{C} 31-\mathrm{H} 31 \mathrm{C}$ & 109.5 \\
\hline $\mathrm{S} 31-\mathrm{C} 32-\mathrm{H} 32 \mathrm{~A}$ & 109.5 \\
\hline $\mathrm{S} 31-\mathrm{C} 32-\mathrm{H} 32 \mathrm{~B}$ & 109.5 \\
\hline $\mathrm{H} 32 \mathrm{~A}-\mathrm{C} 32-\mathrm{H} 32 \mathrm{~B}$ & 109.5 \\
\hline $\mathrm{S} 31-\mathrm{C} 32-\mathrm{H} 32 \mathrm{C}$ & 109.5 \\
\hline
\end{tabular}




\begin{tabular}{|c|c|}
\hline $\mathrm{N} 14-\mathrm{C} 13-\mathrm{C} 12$ & $109.72(19)$ \\
\hline $\mathrm{N} 14-\mathrm{C} 13-\mathrm{H} 13 \mathrm{~A}$ & 109.7 \\
\hline $\mathrm{C} 12-\mathrm{C} 13-\mathrm{H} 13 \mathrm{~A}$ & 109.7 \\
\hline $\mathrm{N} 14-\mathrm{C} 13-\mathrm{H} 13 \mathrm{~B}$ & 109.7 \\
\hline $\mathrm{C} 12-\mathrm{C} 13-\mathrm{H} 13 \mathrm{~B}$ & 109.7 \\
\hline $\mathrm{H} 13 \mathrm{~A}-\mathrm{C} 13-\mathrm{H} 13 \mathrm{~B}$ & 108.2 \\
\hline $\mathrm{C} 17-\mathrm{N} 14-\mathrm{C} 15$ & $112.1(2)$ \\
\hline $\mathrm{C} 17-\mathrm{N} 14-\mathrm{C} 13$ & $112.7(2)$ \\
\hline $\mathrm{C} 15-\mathrm{N} 14-\mathrm{C} 13$ & $109.17(18)$ \\
\hline $\mathrm{C} 17-\mathrm{N} 14-\mathrm{H} 14$ & $104.9(16)$ \\
\hline $\mathrm{C} 15-\mathrm{N} 14-\mathrm{H} 14$ & $110.5(16)$ \\
\hline $\mathrm{C} 13-\mathrm{N} 14-\mathrm{H} 14$ & $107.3(16)$ \\
\hline $\mathrm{N} 14-\mathrm{C} 15-\mathrm{C} 16$ & $110.49(19)$ \\
\hline $\mathrm{N} 14-\mathrm{C} 15-\mathrm{H} 15 \mathrm{~A}$ & 109.6 \\
\hline $\mathrm{C} 16-\mathrm{C} 15-\mathrm{H} 15 \mathrm{~A}$ & 109.6 \\
\hline $\mathrm{N} 14-\mathrm{C} 15-\mathrm{H} 15 \mathrm{~B}$ & 109.6 \\
\hline $\mathrm{C} 16-\mathrm{C} 15-\mathrm{H} 15 \mathrm{~B}$ & 109.6 \\
\hline $\mathrm{H} 15 \mathrm{~A}-\mathrm{C} 15-\mathrm{H} 15 \mathrm{~B}$ & 108.1 \\
\hline $\mathrm{C} 11 \mathrm{~A}-\mathrm{C} 1-\mathrm{C} 2-\mathrm{C} 3$ & $1.1(4)$ \\
\hline $\mathrm{C} 1-\mathrm{C} 2-\mathrm{C} 3-\mathrm{C} 4$ & $-5.1(4)$ \\
\hline $\mathrm{C} 2-\mathrm{C} 3-\mathrm{C} 4-\mathrm{C} 4 \mathrm{~A}$ & $3.6(4)$ \\
\hline $\mathrm{C} 3-\mathrm{C} 4-\mathrm{C} 4 \mathrm{~A}-\mathrm{C} 11 \mathrm{~A}$ & $1.8(4)$ \\
\hline $\mathrm{C} 3-\mathrm{C} 4-\mathrm{C} 4 \mathrm{~A}-\mathrm{N} 5$ & $-178.5(2)$ \\
\hline $\mathrm{C} 4-\mathrm{C} 4 \mathrm{~A}-\mathrm{N} 5-\mathrm{C} 5 \mathrm{~A}$ & $115.1(3)$ \\
\hline $\mathrm{C} 11 \mathrm{~A}-\mathrm{C} 4 \mathrm{~A}-\mathrm{N} 5-\mathrm{C} 5 \mathrm{~A}$ & $-65.2(3)$ \\
\hline $\mathrm{C} 4 \mathrm{~A}-\mathrm{N} 5-\mathrm{C} 5 \mathrm{~A}-\mathrm{C} 6$ & $-117.8(2)$ \\
\hline $\mathrm{C} 4 \mathrm{~A}-\mathrm{N} 5-\mathrm{C} 5 \mathrm{~A}-\mathrm{C} 9 \mathrm{~A}$ & $63.5(3)$ \\
\hline $\mathrm{C} 9 \mathrm{~A}-\mathrm{C} 5 \mathrm{~A}-\mathrm{C} 6-\mathrm{C} 7$ & $-0.1(4)$ \\
\hline $\mathrm{N} 5-\mathrm{C} 5 \mathrm{~A}-\mathrm{C} 6-\mathrm{C} 7$ & $-178.8(2)$ \\
\hline $\mathrm{C} 5 \mathrm{~A}-\mathrm{C} 6-\mathrm{C} 7-\mathrm{C} 8$ & $-1.9(4)$ \\
\hline $\mathrm{C} 6-\mathrm{C} 7-\mathrm{C} 8-\mathrm{C} 9$ & $2.3(4)$ \\
\hline $\mathrm{C} 6-\mathrm{C} 7-\mathrm{C} 8-\mathrm{Cl} 8$ & $-177.8(2)$ \\
\hline $\mathrm{C} 7-\mathrm{C} 8-\mathrm{C} 9-\mathrm{C} 9 \mathrm{~A}$ & $-0.8(4)$ \\
\hline $\mathrm{C} 18-\mathrm{C} 8-\mathrm{C} 9-\mathrm{C} 9 \mathrm{~A}$ & $179.38(18)$ \\
\hline $\mathrm{C} 8-\mathrm{C} 9-\mathrm{C} 9 \mathrm{~A}-\mathrm{N} 10$ & $171.3(2)$ \\
\hline $\mathrm{C} 8-\mathrm{C} 9-\mathrm{C} 9 \mathrm{~A}-\mathrm{C} 5 \mathrm{~A}$ & $-1.3(3)$ \\
\hline $\mathrm{C} 6-\mathrm{C} 5 \mathrm{~A}-\mathrm{C} 9 \mathrm{~A}-\mathrm{C} 9$ & $1.7(3)$ \\
\hline $\mathrm{N} 5-\mathrm{C} 5 \mathrm{~A}-\mathrm{C} 9 \mathrm{~A}-\mathrm{C} 9$ & $-179.6(2)$ \\
\hline $\mathrm{C} 6-\mathrm{C} 5 \mathrm{~A}-\mathrm{C} 9 \mathrm{~A}-\mathrm{N} 10$ & $-170.4(2)$ \\
\hline $\mathrm{N} 5-\mathrm{C} 5 \mathrm{~A}-\mathrm{C} 9 \mathrm{~A}-\mathrm{N} 10$ & $8.2(3)$ \\
\hline $\mathrm{C} 9-\mathrm{C} 9 \mathrm{~A}-\mathrm{N} 10-\mathrm{C} 11$ & $146.3(2)$ \\
\hline $\mathrm{C} 5 \mathrm{~A}-\mathrm{C} 9 \mathrm{~A}-\mathrm{N} 10-\mathrm{C} 11$ & $-41.5(3)$ \\
\hline $\mathrm{C} 9 \mathrm{~A}-\mathrm{N} 10-\mathrm{C} 11-\mathrm{N} 11$ & $176.4(2)$ \\
\hline $\mathrm{C} 9 \mathrm{~A}-\mathrm{N} 10-\mathrm{C} 11-\mathrm{C} 11 \mathrm{~A}$ & $-7.9(4)$ \\
\hline $\mathrm{C} 2-\mathrm{C} 1-\mathrm{C} 11 \mathrm{~A}-\mathrm{C} 4 \mathrm{~A}$ & $4.3(3)$ \\
\hline $\mathrm{C} 2-\mathrm{C} 1-\mathrm{C} 11 \mathrm{~A}-\mathrm{C} 11$ & $-177.0(2)$ \\
\hline $\mathrm{C} 4-\mathrm{C} 4 \mathrm{~A}-\mathrm{C} 11 \mathrm{~A}-\mathrm{C} 1$ & $-5.7(3)$ \\
\hline
\end{tabular}

$\begin{array}{ll}\mathrm{H} 32 \mathrm{~A}-\mathrm{C} 32-\mathrm{H} 32 \mathrm{C} & 109.5 \\ \mathrm{H} 32 \mathrm{~B}-\mathrm{C} 32-\mathrm{H} 32 \mathrm{C} & 109.5 \\ \mathrm{O} 41-\mathrm{S} 41-\mathrm{C} 42 & 106.5(4) \\ \mathrm{O} 41-\mathrm{S} 41-\mathrm{C} 41 & 108.6(5) \\ \mathrm{C} 42-\mathrm{S} 41-\mathrm{C} 41 & 99.8(5) \\ \mathrm{S} 41-\mathrm{C} 41-\mathrm{H} 41 \mathrm{~A} & 109.5 \\ \mathrm{~S} 41-\mathrm{C} 41-\mathrm{H} 41 \mathrm{~B} & 109.5 \\ \mathrm{H} 41 \mathrm{~A}-\mathrm{C} 41-\mathrm{H} 41 \mathrm{~B} & 109.5 \\ \mathrm{~S} 41-\mathrm{C} 41-\mathrm{H} 41 \mathrm{C} & 109.5 \\ \mathrm{H} 41 \mathrm{~A}-\mathrm{C} 41-\mathrm{H} 41 \mathrm{C} & 109.5 \\ \mathrm{H} 41 \mathrm{~B}-\mathrm{C} 41-\mathrm{H} 41 \mathrm{C} & 109.5 \\ \mathrm{~S} 41-\mathrm{C} 42-\mathrm{H} 42 \mathrm{~A} & 109.5 \\ \text { S41-C42-H42B } & 109.5 \\ \mathrm{H} 42 \mathrm{~A}-\mathrm{C} 42-\mathrm{H} 42 \mathrm{~B} & 109.5 \\ \text { S41-C42-H42C } & 109.5 \\ \text { H42A-C42-H42C } & 109.5 \\ \text { H42B-C42-H42C } & 109.5 \\ & \end{array}$

$\mathrm{C} 11 \mathrm{~A}-\mathrm{C} 11-\mathrm{N} 11-\mathrm{C} 16 \quad 16.8(3)$

$\mathrm{N} 10-\mathrm{C} 11-\mathrm{N} 11-\mathrm{C} 12$

$\mathrm{C} 11 \mathrm{~A}-\mathrm{C} 11-\mathrm{N} 11-\mathrm{C} 12 \quad-169.7$ (2)

$\mathrm{C} 11-\mathrm{N} 11-\mathrm{C} 12-\mathrm{C} 13 \quad-120.8(2)$

$\mathrm{C} 16-\mathrm{N} 11-\mathrm{C} 12-\mathrm{C} 13 \quad 53.4(3)$

$\mathrm{N} 11-\mathrm{C} 12-\mathrm{C} 13-\mathrm{N} 14 \quad-56.7$ (3)

$\mathrm{C} 12-\mathrm{C} 13-\mathrm{N} 14-\mathrm{C} 17 \quad-175.3$ (2)

$\mathrm{C} 12-\mathrm{C} 13-\mathrm{N} 14-\mathrm{C} 15 \quad 59.5$ (2)

C17-N14-C15-C16 175.4 (2)

$\mathrm{C} 13-\mathrm{N} 14-\mathrm{C} 15-\mathrm{C} 16 \quad-59.1$ (2)

$\mathrm{C} 11-\mathrm{N} 11-\mathrm{C} 16-\mathrm{C} 15 \quad 121.5$ (2)

$\mathrm{C} 12-\mathrm{N} 11-\mathrm{C} 16-\mathrm{C} 15 \quad-52.4(3)$

$\mathrm{N} 14-\mathrm{C} 15-\mathrm{C} 16-\mathrm{N} 11 \quad 55.3(3)$

$\mathrm{C} 26-\mathrm{C} 21-\mathrm{C} 22-\mathrm{C} 23 \quad-0.1(3)$

$\mathrm{C} 27-\mathrm{C} 21-\mathrm{C} 22-\mathrm{C} 23 \quad-177.5$ (2)

$\mathrm{C} 21-\mathrm{C} 22-\mathrm{C} 23-\mathrm{C} 24 \quad-0.4$ (4)

$\mathrm{C} 21-\mathrm{C} 22-\mathrm{C} 23-\mathrm{N} 23 \quad 176.6(2)$

$\mathrm{C} 22-\mathrm{C} 23-\mathrm{C} 24-\mathrm{C} 25 \quad 0.3(4)$

$\mathrm{N} 23-\mathrm{C} 23-\mathrm{C} 24-\mathrm{C} 25 \quad-176.7$ (2)

$\mathrm{C} 23-\mathrm{C} 24-\mathrm{C} 25-\mathrm{C} 26 \quad 0.3$ (4)

$\mathrm{C} 23-\mathrm{C} 24-\mathrm{C} 25-\mathrm{N} 25 \quad 177.4$ (2)

$\mathrm{C} 24-\mathrm{C} 25-\mathrm{C} 26-\mathrm{C} 21 \quad-0.7$ (3)

$\mathrm{N} 25-\mathrm{C} 25-\mathrm{C} 26-\mathrm{C} 21 \quad-177.8$ (2)

$\mathrm{C} 22-\mathrm{C} 21-\mathrm{C} 26-\mathrm{C} 25 \quad 0.6(3)$

$\mathrm{C} 27-\mathrm{C} 21-\mathrm{C} 26-\mathrm{C} 25 \quad 178.1$ (2)

$\mathrm{C} 22-\mathrm{C} 21-\mathrm{C} 27-\mathrm{O} 21 \quad 177.5$ (2)

$\mathrm{C} 26-\mathrm{C} 21-\mathrm{C} 27-\mathrm{O} 21 \quad 0.0(3)$

$\mathrm{C} 22-\mathrm{C} 21-\mathrm{C} 27-\mathrm{O} 22 \quad-2.0$ (3)

$\mathrm{C} 26-\mathrm{C} 21-\mathrm{C} 27-\mathrm{O} 22 \quad-179.5$ (2) 


$\begin{array}{llll}\mathrm{N} 5-\mathrm{C} 4 \mathrm{~A}-\mathrm{C} 11 \mathrm{~A}-\mathrm{C} 1 & 174.6(2) & \mathrm{C} 24-\mathrm{C} 23-\mathrm{N} 23-\mathrm{O} 24 & -0.8(4) \\ \mathrm{C} 4-\mathrm{C} 4 \mathrm{~A}-\mathrm{C} 11 \mathrm{~A}-\mathrm{C} 11 & 175.5(2) & \mathrm{C} 22-\mathrm{C} 23-\mathrm{N} 23-\mathrm{O} 24 & -177.9(3) \\ \mathrm{N} 5-\mathrm{C} 4 \mathrm{~A}-\mathrm{C} 11 \mathrm{~A}-\mathrm{C} 11 & -4.1(3) & \mathrm{C} 24-\mathrm{C} 23-\mathrm{N} 23-\mathrm{O} 23 & 177.6(3) \\ \mathrm{N} 10-\mathrm{C} 11-\mathrm{C} 11 \mathrm{~A}-\mathrm{C} 1 & -129.2(3) & \mathrm{C} 22-\mathrm{C} 23-\mathrm{N} 23-\mathrm{O} 23 & 0.5(4) \\ \mathrm{N} 11-\mathrm{C} 11-\mathrm{C} 11 \mathrm{~A}-\mathrm{C} 1 & 46.5(3) & \mathrm{C} 24-\mathrm{C} 25-\mathrm{N} 25-\mathrm{O} 26 & -178.3(2) \\ \mathrm{N} 10-\mathrm{C} 11-\mathrm{C} 11 \mathrm{~A}-\mathrm{C} 4 \mathrm{~A} & 49.5(3) & \mathrm{C} 26-\mathrm{C} 25-\mathrm{N} 25-\mathrm{O} 26 & -1.1(4) \\ \mathrm{N} 11-\mathrm{C} 11-\mathrm{C} 11 \mathrm{~A}-\mathrm{C} 4 \mathrm{~A} & -134.7(2) & \mathrm{C} 24-\mathrm{C} 25-\mathrm{N} 25-\mathrm{O} 25 & 1.6(4) \\ \mathrm{N} 10-\mathrm{C} 11-\mathrm{N} 11-\mathrm{C} 16 & -167.1(2) & \mathrm{C} 26-\mathrm{C} 25-\mathrm{N} 25-\mathrm{O} 25 & 178.8(3)\end{array}$

Hydrogen-bond geometry $\left(\AA,{ }^{\circ}\right)$

\begin{tabular}{lllll}
\hline$D-\mathrm{H} \cdots A$ & $D-\mathrm{H}$ & $\mathrm{H} \cdots A$ & $D \cdots A$ & $D-\mathrm{H} \cdots A$ \\
\hline $\mathrm{N} 5-\mathrm{H} 5 \cdots \mathrm{O} 31$ & $0.83(3)$ & $2.10(3)$ & $2.921(4)$ & $170(3)$ \\
$\mathrm{N} 14-\mathrm{H} 14 \cdots \mathrm{O} 22$ & $1.00(3)$ & $1.58(3)$ & $2.575(3)$ & $176(2)$ \\
$\mathrm{C} 4-\mathrm{H} 4 \cdots \mathrm{O} 31$ & 0.95 & 2.58 & $3.342(4)$ & 137 \\
$\mathrm{C} 4-\mathrm{H} 4 \cdots \mathrm{O} 41$ & 0.95 & 2.38 & $3.208(7)$ & 146 \\
$\mathrm{C} 6-\mathrm{H} 6 \cdots \mathrm{O} 31$ & 0.95 & 2.54 & $3.313(5)$ & 139
\end{tabular}

(II) Clozapinium hydrogen maleate 0.21-hydrate

Crystal data

$\mathrm{C}_{18} \mathrm{H}_{20} \mathrm{ClN}_{4}^{+} \cdot \mathrm{C}_{4} \mathrm{H}_{3} \mathrm{O}_{4}^{-} \cdot 0.21 \mathrm{H}_{2} \mathrm{O}$

$M_{r}=446.68$

Monoclinic, $P 2{ }_{1} / c$

$a=9.7166(3) \AA$

$b=9.9699(2) \AA$

$c=23.1059(6) \AA$

$\beta=96.800(3)^{\circ}$

$V=2222.60(10) \AA^{3}$

$Z=4$

\section{Data collection}

Agilent Eos Gemini

diffractometer

Radiation source: Enhance $(\mathrm{Cu}) \mathrm{X}$-ray Source

$\omega$ scans

Absorption correction: multi-scan

(CrysAlis RED; Agilent, 2012)

$T_{\min }=0.440, T_{\max }=0.668$

8634 measured reflections

\section{Refinement}

Refinement on $F^{2}$

Least-squares matrix: full

$R\left[F^{2}>2 \sigma\left(F^{2}\right)\right]=0.048$

$w R\left(F^{2}\right)=0.133$

$S=1.03$

4228 reflections

295 parameters

0 restraints
$F(000)=936.4$

$D_{\mathrm{x}}=1.335 \mathrm{Mg} \mathrm{m}^{-3}$

$\mathrm{Cu} K \alpha$ radiation, $\lambda=1.54184 \AA$

Cell parameters from 4228 reflections

$\theta=3.9-71.2^{\circ}$

$\mu=1.84 \mathrm{~mm}^{-1}$

$T=173 \mathrm{~K}$

Block, colourless

$0.46 \times 0.32 \times 0.22 \mathrm{~mm}$

4228 independent reflections

3552 reflections with $I>2 \sigma(I)$

$R_{\text {int }}=0.026$

$\theta_{\text {max }}=71.2^{\circ}, \theta_{\min }=3.9^{\circ}$

$h=-11 \rightarrow 11$

$k=-12 \rightarrow 7$

$l=-28 \rightarrow 27$

Hydrogen site location: difference Fourier map

$\mathrm{H}$ atoms treated by a mixture of independent and constrained refinement

$w=1 /\left[\sigma^{2}\left(F_{0}^{2}\right)+(0.069 P)^{2}+0.8133 P\right]$

where $P=\left(F_{\mathrm{o}}^{2}+2 F_{\mathrm{c}}{ }^{2}\right) / 3$

$(\Delta / \sigma)_{\max }<0.001$

$\Delta \rho_{\max }=0.46 \mathrm{e}^{-3}$

$\Delta \rho_{\min }=-0.30$ e $\AA^{-3}$ 


\section{Special details}

Geometry. All e.s.d.'s (except the e.s.d. in the dihedral angle between two 1.s. planes) are estimated using the full covariance matrix. The cell e.s.d.'s are taken into account individually in the estimation of e.s.d.'s in distances, angles and torsion angles; correlations between e.s.d.'s in cell parameters are only used when they are defined by crystal symmetry. An approximate (isotropic) treatment of cell e.s.d.'s is used for estimating e.s.d.'s involving l.s. planes.

Fractional atomic coordinates and isotropic or equivalent isotropic displacement parameters $\left(\AA^{2}\right)$

\begin{tabular}{|c|c|c|c|c|c|}
\hline & $x$ & $y$ & $z$ & $U_{\text {iso }} * / U_{\text {eq }}$ & Occ. $(<1)$ \\
\hline $\mathrm{C} 1$ & $0.6656(2)$ & $0.4997(2)$ & $0.23723(9)$ & $0.0361(4)$ & \\
\hline H1 & 0.7048 & 0.4466 & 0.2691 & $0.043^{*}$ & \\
\hline $\mathrm{C} 2$ & $0.7438(2)$ & $0.5303(2)$ & $0.19279(9)$ & $0.0417(5)$ & \\
\hline $\mathrm{H} 2$ & 0.8362 & 0.4981 & 0.1940 & $0.050 *$ & \\
\hline $\mathrm{C} 3$ & $0.6866(2)$ & $0.6083(2)$ & $0.14640(9)$ & $0.0409(5)$ & \\
\hline $\mathrm{H} 3$ & 0.7417 & 0.6340 & 0.1169 & $0.049 *$ & \\
\hline $\mathrm{C} 4$ & $0.5503(2)$ & $0.6488(2)$ & $0.14272(8)$ & $0.0356(4)$ & \\
\hline $\mathrm{H} 4$ & 0.5110 & 0.6994 & 0.1100 & $0.043 *$ & \\
\hline $\mathrm{C} 4 \mathrm{~A}$ & 0.46942 (19) & 0.61605 (19) & $0.18684(8)$ & $0.0291(4)$ & \\
\hline N5 & $0.32745(17)$ & $0.65240(17)$ & $0.18232(7)$ & $0.0326(3)$ & \\
\hline H5 & $0.307(2)$ & $0.703(3)$ & $0.1527(11)$ & $0.039^{*}$ & \\
\hline $\mathrm{C} 5 \mathrm{~A}$ & $0.23552(18)$ & $0.54101(19)$ & $0.18333(8)$ & $0.0296(4)$ & \\
\hline C6 & $0.1401(2)$ & $0.5111(2)$ & $0.13518(8)$ & $0.0357(4)$ & \\
\hline H6 & 0.1364 & 0.5660 & 0.1014 & $0.043^{*}$ & \\
\hline $\mathrm{C} 7$ & $0.0501(2)$ & $0.4028(2)$ & $0.13556(9)$ & $0.0385(5)$ & \\
\hline $\mathrm{H} 7$ & -0.0150 & 0.3835 & 0.1026 & $0.046^{*}$ & \\
\hline $\mathrm{C} 8$ & $0.05780(19)$ & $0.3239(2)$ & $0.18497(9)$ & $0.0360(4)$ & \\
\hline $\mathrm{Cl} 8$ & $-0.05340(6)$ & $0.18656(6)$ & $0.18694(3)$ & $0.0560(2)$ & \\
\hline C9 & 0.14961 (19) & $0.3526(2)$ & $0.23364(9)$ & $0.0334(4)$ & \\
\hline H9 & 0.1507 & 0.2984 & 0.2675 & $0.040^{*}$ & \\
\hline C9A & $0.24090(18)$ & $0.46068(19)$ & $0.23353(8)$ & $0.0290(4)$ & \\
\hline N10 & $0.32420(16)$ & $0.48876(16)$ & $0.28580(6)$ & $0.0301(3)$ & \\
\hline $\mathrm{C} 11$ & $0.45129(18)$ & $0.52545(18)$ & $0.28708(8)$ & $0.0283(4)$ & \\
\hline C11A & $0.52924(19)$ & $0.54587(19)$ & $0.23581(8)$ & $0.0293(4)$ & \\
\hline N11 & $0.52983(16)$ & $0.53872(17)$ & $0.34067(6)$ & $0.0315(4)$ & \\
\hline $\mathrm{C} 12$ & $0.46621(19)$ & $0.50463(19)$ & $0.39301(7)$ & $0.0300(4)$ & \\
\hline $\mathrm{H} 12 \mathrm{~A}$ & 0.5381 & 0.4687 & 0.4229 & $0.036^{*}$ & \\
\hline H12B & 0.3960 & 0.4336 & 0.3834 & $0.036^{*}$ & \\
\hline $\mathrm{C} 13$ & $0.39818(18)$ & $0.62443(19)$ & $0.41749(8)$ & $0.0302(4)$ & \\
\hline H13A & 0.3214 & 0.6569 & 0.3889 & $0.036^{*}$ & \\
\hline H13B & 0.3592 & 0.5986 & 0.4536 & $0.036^{*}$ & \\
\hline N14 & $0.50291(17)$ & $0.73320(17)$ & $0.43053(7)$ & $0.0324(4)$ & \\
\hline H14 & $0.565(3)$ & $0.702(2)$ & $0.4574(11)$ & $0.039 *$ & \\
\hline $\mathrm{C} 15$ & $0.5702(2)$ & $0.7683(2)$ & $0.37746(8)$ & $0.0382(5)$ & \\
\hline H15A & 0.6437 & 0.8358 & 0.3878 & $0.046^{*}$ & \\
\hline H15B & 0.5004 & 0.8082 & 0.3477 & $0.046^{*}$ & \\
\hline $\mathrm{C} 16$ & $0.63243(19)$ & $0.6451(2)$ & $0.35254(8)$ & $0.0361(4)$ & \\
\hline H16A & 0.6701 & 0.6694 & 0.3160 & $0.043^{*}$ & \\
\hline $\mathrm{H} 16 \mathrm{~B}$ & 0.7101 & 0.6120 & 0.3805 & $0.043^{*}$ & \\
\hline
\end{tabular}




\begin{tabular}{|c|c|c|c|c|c|}
\hline $\mathrm{C} 17$ & $0.4417(3)$ & $0.8518(2)$ & $0.45611(11)$ & $0.0561(6)$ & \\
\hline H17A & 0.3690 & 0.8896 & 0.4278 & $0.084 *$ & \\
\hline H17B & 0.5140 & 0.9193 & 0.4661 & $0.084 *$ & \\
\hline $\mathrm{H} 17 \mathrm{C}$ & 0.4017 & 0.8251 & 0.4914 & $0.084 *$ & \\
\hline $\mathrm{C} 21$ & $1.1468(2)$ & $0.6834(2)$ & $0.54450(9)$ & $0.0415(5)$ & \\
\hline $\mathrm{O} 21$ & $1.1086(2)$ & $0.7228(3)$ & $0.49282(8)$ & $0.0910(9)$ & \\
\hline $\mathrm{H} 21$ & $1.006(5)$ & $0.739(5)$ & $0.488(2)$ & $0.137^{*}$ & \\
\hline $\mathrm{O} 22$ & $1.26642(15)$ & $0.69322(19)$ & $0.56647(7)$ & $0.0533(4)$ & \\
\hline $\mathrm{C} 22$ & $1.0433(2)$ & $0.6232(3)$ & $0.57919(10)$ & $0.0490(6)$ & \\
\hline $\mathrm{H} 22$ & 1.0817 & 0.5844 & 0.6151 & $0.059^{*}$ & \\
\hline $\mathrm{C} 23$ & $0.9056(2)$ & $0.6141(3)$ & $0.56863(10)$ & $0.0494(6)$ & \\
\hline $\mathrm{H} 23$ & 0.8618 & 0.5719 & 0.5984 & $0.059^{*}$ & \\
\hline $\mathrm{C} 24$ & $0.8103(2)$ & $0.6601(2)$ & $0.51734(8)$ & $0.0356(4)$ & \\
\hline $\mathrm{O} 23$ & $0.68470(15)$ & $0.6486(2)$ & $0.51985(7)$ & $0.0526(4)$ & \\
\hline $\mathrm{O} 24$ & $0.85884(18)$ & $0.7068(3)$ & $0.47375(8)$ & $0.0810(8)$ & \\
\hline $\mathrm{O} 31$ & $0.7719(11)$ & $0.9745(11)$ & $0.5046(5)$ & $0.074(4)^{*}$ & $0.210(7)$ \\
\hline H31A & 0.8183 & 0.9016 & 0.4951 & $0.111^{*}$ & $0.210(7)$ \\
\hline H31B & 0.8247 & 1.0490 & 0.5081 & $0.111^{*}$ & 0.210 \\
\hline
\end{tabular}

Atomic displacement parameters $\left(\AA^{2}\right)$

\begin{tabular}{lllllll}
\hline & $U^{11}$ & $U^{22}$ & $U^{33}$ & $U^{12}$ & $U^{13}$ & $U^{23}$ \\
\hline C1 & $0.0326(9)$ & $0.0429(11)$ & $0.0324(9)$ & $0.0035(8)$ & $0.0016(7)$ & $-0.0027(8)$ \\
C2 & $0.0333(10)$ & $0.0513(13)$ & $0.0421(11)$ & $0.0024(9)$ & $0.0106(8)$ & $-0.0098(10)$ \\
C3 & $0.0439(11)$ & $0.0483(12)$ & $0.0328(10)$ & $-0.0098(9)$ & $0.0148(8)$ & $-0.0075(9)$ \\
C4 & $0.0435(11)$ & $0.0355(10)$ & $0.0273(9)$ & $-0.0063(8)$ & $0.0031(8)$ & $-0.0016(8)$ \\
C4A & $0.0319(9)$ & $0.0278(9)$ & $0.0271(8)$ & $-0.0028(7)$ & $0.0018(7)$ & $-0.0056(7)$ \\
N5 & $0.0350(8)$ & $0.0308(8)$ & $0.0308(8)$ & $0.0020(7)$ & $-0.0011(6)$ & $0.0024(7)$ \\
C5A & $0.0259(8)$ & $0.0304(9)$ & $0.0321(9)$ & $0.0043(7)$ & $0.0013(7)$ & $-0.0053(7)$ \\
C6 & $0.0331(9)$ & $0.0416(11)$ & $0.0310(9)$ & $0.0058(8)$ & $-0.0016(7)$ & $-0.0024(8)$ \\
C7 & $0.0277(9)$ & $0.0469(12)$ & $0.0388(10)$ & $0.0021(8)$ & $-0.0047(8)$ & $-0.0118(9)$ \\
C8 & $0.0250(9)$ & $0.0357(10)$ & $0.0467(11)$ & $-0.0019(8)$ & $0.0017(8)$ & $-0.0088(9)$ \\
C18 & $0.0448(3)$ & $0.0505(3)$ & $0.0690(4)$ & $-0.0191(2)$ & $-0.0089(3)$ & $-0.0033(3)$ \\
C9 & $0.0279(9)$ & $0.0355(10)$ & $0.0365(9)$ & $0.0002(8)$ & $0.0022(7)$ & $-0.0017(8)$ \\
C9A & $0.0245(8)$ & $0.0322(9)$ & $0.0301(9)$ & $0.0035(7)$ & $0.0020(7)$ & $-0.0059(7)$ \\
N10 & $0.0299(8)$ & $0.0341(8)$ & $0.0261(7)$ & $-0.0022(6)$ & $0.0020(6)$ & $-0.0023(6)$ \\
C11 & $0.0294(9)$ & $0.0293(9)$ & $0.0258(8)$ & $0.0004(7)$ & $0.0016(7)$ & $-0.0011(7)$ \\
C11A & $0.0297(9)$ & $0.0325(9)$ & $0.0254(8)$ & $-0.0012(7)$ & $0.0028(7)$ & $-0.0038(7)$ \\
N11 & $0.0308(8)$ & $0.0407(9)$ & $0.0227(7)$ & $-0.0074(7)$ & $0.0025(6)$ & $0.0006(6)$ \\
C12 & $0.0341(9)$ & $0.0316(9)$ & $0.0237(8)$ & $-0.0051(7)$ & $0.0012(7)$ & $0.0029(7)$ \\
C13 & $0.0274(8)$ & $0.0358(10)$ & $0.0268(8)$ & $-0.0034(7)$ & $0.0001(7)$ & $0.0030(7)$ \\
N14 & $0.0388(9)$ & $0.0302(8)$ & $0.0265(7)$ & $-0.0031(7)$ & $-0.0026(6)$ & $0.0028(6)$ \\
C15 & $0.0458(11)$ & $0.0390(11)$ & $0.0281(9)$ & $-0.0157(9)$ & $-0.0022(8)$ & $0.0080(8)$ \\
C16 & $0.0297(9)$ & $0.0537(12)$ & $0.0241(8)$ & $-0.0129(9)$ & $-0.0004(7)$ & $0.0012(8)$ \\
C17 & $0.0829(18)$ & $0.0339(11)$ & $0.0526(14)$ & $0.0015(12)$ & $0.0122(13)$ & $-0.0047(10)$ \\
C21 & $0.0347(10)$ & $0.0498(12)$ & $0.0383(10)$ & $-0.0006(9)$ & $-0.0032(8)$ & $-0.0123(9)$ \\
O21 & $0.0376(9)$ & $0.183(3)$ & $0.0495(10)$ & $-0.0273(13)$ & $-0.0053(8)$ & $0.0325(14)$ \\
O22 & $0.0315(8)$ & $0.0719(12)$ & $0.0541(9)$ & $0.0031(7)$ & $-0.0052(7)$ & $-0.0144(8)$ \\
& & & & &
\end{tabular}




\begin{tabular}{lllllll}
$\mathrm{C} 22$ & $0.0418(11)$ & $0.0594(15)$ & $0.0421(11)$ & $0.0017(10)$ & $-0.0102(9)$ & $0.0144(10)$ \\
$\mathrm{C} 23$ & $0.0419(11)$ & $0.0644(15)$ & $0.0401(11)$ & $-0.0090(11)$ & $-0.0029(9)$ & $0.0215(11)$ \\
$\mathrm{C} 24$ & $0.0354(10)$ & $0.0418(11)$ & $0.0278(9)$ & $-0.0107(9)$ & $-0.0041(7)$ & $0.0051(8)$ \\
$\mathrm{O} 23$ & $0.0344(8)$ & $0.0847(13)$ & $0.0367(8)$ & $-0.0113(8)$ & $-0.0040(6)$ & $0.0215(8)$ \\
$\mathrm{O} 24$ & $0.0400(9)$ & $0.158(2)$ & $0.0418(9)$ & $-0.0252(11)$ & $-0.0089(7)$ & $0.0419(12)$ \\
\hline
\end{tabular}

Geometric parameters $\left(\hat{A},{ }^{\circ}\right)$

\begin{tabular}{|c|c|c|c|}
\hline $\mathrm{C} 1-\mathrm{C} 2$ & $1.382(3)$ & $\mathrm{C} 12-\mathrm{C} 13$ & $1.507(3)$ \\
\hline $\mathrm{C} 1-\mathrm{C} 11 \mathrm{~A}$ & $1.399(3)$ & $\mathrm{C} 12-\mathrm{H} 12 \mathrm{~A}$ & 0.9900 \\
\hline $\mathrm{C} 1-\mathrm{H} 1$ & 0.9500 & $\mathrm{C} 12-\mathrm{H} 12 \mathrm{~B}$ & 0.9900 \\
\hline $\mathrm{C} 2-\mathrm{C} 3$ & $1.387(3)$ & $\mathrm{C} 13-\mathrm{N} 14$ & $1.493(2)$ \\
\hline $\mathrm{C} 2-\mathrm{H} 2$ & 0.9500 & $\mathrm{C} 13-\mathrm{H} 13 \mathrm{~A}$ & 0.9900 \\
\hline $\mathrm{C} 3-\mathrm{C} 4$ & $1.377(3)$ & C13-H13B & 0.9900 \\
\hline $\mathrm{C} 3-\mathrm{H} 3$ & 0.9500 & $\mathrm{~N} 14-\mathrm{C} 17$ & $1.478(3)$ \\
\hline $\mathrm{C} 4-\mathrm{C} 4 \mathrm{~A}$ & $1.397(3)$ & $\mathrm{N} 14-\mathrm{C} 15$ & $1.498(2)$ \\
\hline $\mathrm{C} 4-\mathrm{H} 4$ & 0.9500 & $\mathrm{~N} 14-\mathrm{H} 14$ & $0.87(3)$ \\
\hline $\mathrm{C} 4 \mathrm{~A}-\mathrm{C} 11 \mathrm{~A}$ & $1.397(3)$ & $\mathrm{C} 15-\mathrm{C} 16$ & $1.513(3)$ \\
\hline $\mathrm{C} 4 \mathrm{~A}-\mathrm{N} 5$ & $1.418(2)$ & $\mathrm{C} 15-\mathrm{H} 15 \mathrm{~A}$ & 0.9900 \\
\hline $\mathrm{N} 5-\mathrm{C} 5 \mathrm{~A}$ & $1.427(3)$ & C15-H15B & 0.9900 \\
\hline N5-H5 & $0.86(3)$ & C16-H16A & 0.9900 \\
\hline $\mathrm{C} 5 \mathrm{~A}-\mathrm{C} 6$ & $1.394(3)$ & C16-H16B & 0.9900 \\
\hline $\mathrm{C} 5 \mathrm{~A}-\mathrm{C} 9 \mathrm{~A}$ & $1.405(3)$ & C17-H17A & 0.9800 \\
\hline $\mathrm{C} 6-\mathrm{C} 7$ & $1.390(3)$ & C17-H17B & 0.9800 \\
\hline $\mathrm{C} 6-\mathrm{H} 6$ & 0.9500 & $\mathrm{C} 17-\mathrm{H} 17 \mathrm{C}$ & 0.9800 \\
\hline $\mathrm{C} 7-\mathrm{C} 8$ & $1.381(3)$ & $\mathrm{C} 21-\mathrm{O} 22$ & $1.216(3)$ \\
\hline $\mathrm{C} 7-\mathrm{H} 7$ & 0.9500 & $\mathrm{C} 21-\mathrm{O} 21$ & $1.270(3)$ \\
\hline $\mathrm{C} 8-\mathrm{C} 9$ & $1.380(3)$ & $\mathrm{C} 21-\mathrm{C} 22$ & $1.485(3)$ \\
\hline $\mathrm{C} 8-\mathrm{Cl} 8$ & $1.748(2)$ & $\mathrm{O} 21-\mathrm{H} 21$ & $1.00(5)$ \\
\hline C9-C9A & $1.396(3)$ & $\mathrm{C} 22-\mathrm{C} 23$ & $1.334(3)$ \\
\hline $\mathrm{C} 9-\mathrm{H} 9$ & 0.9500 & $\mathrm{C} 22-\mathrm{H} 22$ & 0.9500 \\
\hline $\mathrm{C} 9 \mathrm{~A}-\mathrm{N} 10$ & $1.400(2)$ & $\mathrm{C} 23-\mathrm{C} 24$ & $1.487(3)$ \\
\hline $\mathrm{N} 10-\mathrm{C} 11$ & $1.285(2)$ & $\mathrm{C} 23-\mathrm{H} 23$ & 0.9500 \\
\hline $\mathrm{C} 11-\mathrm{N} 11$ & $1.382(2)$ & $\mathrm{C} 24-\mathrm{O} 23$ & $1.234(2)$ \\
\hline $\mathrm{C} 11-\mathrm{C} 11 \mathrm{~A}$ & $1.494(2)$ & $\mathrm{C} 24-\mathrm{O} 24$ & $1.251(3)$ \\
\hline $\mathrm{N} 11-\mathrm{C} 16$ & $1.459(2)$ & $\mathrm{O} 31-\mathrm{H} 31 \mathrm{~A}$ & 0.8959 \\
\hline $\mathrm{N} 11-\mathrm{C} 12$ & $1.462(2)$ & $\mathrm{O} 31-\mathrm{H} 31 \mathrm{~B}$ & 0.9007 \\
\hline $\mathrm{C} 2-\mathrm{C} 1-\mathrm{C} 11 \mathrm{~A}$ & $120.84(19)$ & $\mathrm{N} 11-\mathrm{C} 12-\mathrm{H} 12 \mathrm{~B}$ & 109.2 \\
\hline $\mathrm{C} 2-\mathrm{C} 1-\mathrm{H} 1$ & 119.6 & $\mathrm{C} 13-\mathrm{C} 12-\mathrm{H} 12 \mathrm{~B}$ & 109.2 \\
\hline $\mathrm{C} 11 \mathrm{~A}-\mathrm{C} 1-\mathrm{H} 1$ & 119.6 & $\mathrm{H} 12 \mathrm{~A}-\mathrm{C} 12-\mathrm{H} 12 \mathrm{~B}$ & 107.9 \\
\hline $\mathrm{C} 1-\mathrm{C} 2-\mathrm{C} 3$ & $119.49(19)$ & $\mathrm{N} 14-\mathrm{C} 13-\mathrm{C} 12$ & $109.42(15)$ \\
\hline $\mathrm{C} 1-\mathrm{C} 2-\mathrm{H} 2$ & 120.3 & $\mathrm{~N} 14-\mathrm{C} 13-\mathrm{H} 13 \mathrm{~A}$ & 109.8 \\
\hline $\mathrm{C} 3-\mathrm{C} 2-\mathrm{H} 2$ & 120.3 & $\mathrm{C} 12-\mathrm{C} 13-\mathrm{H} 13 \mathrm{~A}$ & 109.8 \\
\hline $\mathrm{C} 4-\mathrm{C} 3-\mathrm{C} 2$ & $120.47(18)$ & $\mathrm{N} 14-\mathrm{C} 13-\mathrm{H} 13 \mathrm{~B}$ & 109.8 \\
\hline $\mathrm{C} 4-\mathrm{C} 3-\mathrm{H} 3$ & 119.8 & $\mathrm{C} 12-\mathrm{C} 13-\mathrm{H} 13 \mathrm{~B}$ & 109.8 \\
\hline $\mathrm{C} 2-\mathrm{C} 3-\mathrm{H} 3$ & 119.8 & $\mathrm{H} 13 \mathrm{~A}-\mathrm{C} 13-\mathrm{H} 13 \mathrm{~B}$ & 108.2 \\
\hline $\mathrm{C} 3-\mathrm{C} 4-\mathrm{C} 4 \mathrm{~A}$ & $120.39(19)$ & $\mathrm{C} 17-\mathrm{N} 14-\mathrm{C} 13$ & $111.41(17)$ \\
\hline
\end{tabular}




\begin{tabular}{|c|c|c|c|}
\hline $\mathrm{C} 3-\mathrm{C} 4-\mathrm{H} 4$ & 119.8 & $\mathrm{C} 17-\mathrm{N} 14-\mathrm{C} 15$ & $112.05(17)$ \\
\hline $\mathrm{C} 4 \mathrm{~A}-\mathrm{C} 4-\mathrm{H} 4$ & 119.8 & $\mathrm{C} 13-\mathrm{N} 14-\mathrm{C} 15$ & $110.95(14)$ \\
\hline $\mathrm{C} 11 \mathrm{~A}-\mathrm{C} 4 \mathrm{~A}-\mathrm{C} 4$ & $119.47(17)$ & C17-N14-H14 & $106.2(16)$ \\
\hline $\mathrm{C} 11 \mathrm{~A}-\mathrm{C} 4 \mathrm{~A}-\mathrm{N} 5$ & $119.70(16)$ & $\mathrm{C} 13-\mathrm{N} 14-\mathrm{H} 14$ & $106.3(16)$ \\
\hline $\mathrm{C} 4-\mathrm{C} 4 \mathrm{~A}-\mathrm{N} 5$ & $120.83(17)$ & $\mathrm{C} 15-\mathrm{N} 14-\mathrm{H} 14$ & $109.6(16)$ \\
\hline $\mathrm{C} 4 \mathrm{~A}-\mathrm{N} 5-\mathrm{C} 5 \mathrm{~A}$ & $113.90(15)$ & $\mathrm{N} 14-\mathrm{C} 15-\mathrm{C} 16$ & $110.77(16)$ \\
\hline $\mathrm{C} 4 \mathrm{~A}-\mathrm{N} 5-\mathrm{H} 5$ & $110.1(16)$ & $\mathrm{N} 14-\mathrm{C} 15-\mathrm{H} 15 \mathrm{~A}$ & 109.5 \\
\hline $\mathrm{C} 5 \mathrm{~A}-\mathrm{N} 5-\mathrm{H} 5$ & $112.8(17)$ & $\mathrm{C} 16-\mathrm{C} 15-\mathrm{H} 15 \mathrm{~A}$ & 109.5 \\
\hline $\mathrm{C} 6-\mathrm{C} 5 \mathrm{~A}-\mathrm{C} 9 \mathrm{~A}$ & $119.55(18)$ & $\mathrm{N} 14-\mathrm{C} 15-\mathrm{H} 15 \mathrm{~B}$ & 109.5 \\
\hline $\mathrm{C} 6-\mathrm{C} 5 \mathrm{~A}-\mathrm{N} 5$ & $120.79(18)$ & $\mathrm{C} 16-\mathrm{C} 15-\mathrm{H} 15 \mathrm{~B}$ & 109.5 \\
\hline $\mathrm{C} 9 \mathrm{~A}-\mathrm{C} 5 \mathrm{~A}-\mathrm{N} 5$ & $119.66(16)$ & $\mathrm{H} 15 \mathrm{~A}-\mathrm{C} 15-\mathrm{H} 15 \mathrm{~B}$ & 108.1 \\
\hline $\mathrm{C} 7-\mathrm{C} 6-\mathrm{C} 5 \mathrm{~A}$ & $121.36(19)$ & $\mathrm{N} 11-\mathrm{C} 16-\mathrm{C} 15$ & $111.51(16)$ \\
\hline $\mathrm{C} 7-\mathrm{C} 6-\mathrm{H} 6$ & 119.3 & $\mathrm{~N} 11-\mathrm{C} 16-\mathrm{H} 16 \mathrm{~A}$ & 109.3 \\
\hline $\mathrm{C} 5 \mathrm{~A}-\mathrm{C} 6-\mathrm{H} 6$ & 119.3 & $\mathrm{C} 15-\mathrm{C} 16-\mathrm{H} 16 \mathrm{~A}$ & 109.3 \\
\hline $\mathrm{C} 8-\mathrm{C} 7-\mathrm{C} 6$ & $118.39(18)$ & $\mathrm{N} 11-\mathrm{C} 16-\mathrm{H} 16 \mathrm{~B}$ & 109.3 \\
\hline $\mathrm{C} 8-\mathrm{C} 7-\mathrm{H} 7$ & 120.8 & $\mathrm{C} 15-\mathrm{C} 16-\mathrm{H} 16 \mathrm{~B}$ & 109.3 \\
\hline $\mathrm{C} 6-\mathrm{C} 7-\mathrm{H} 7$ & 120.8 & $\mathrm{H} 16 \mathrm{~A}-\mathrm{C} 16-\mathrm{H} 16 \mathrm{~B}$ & 108.0 \\
\hline $\mathrm{C} 9-\mathrm{C} 8-\mathrm{C} 7$ & $121.44(19)$ & N14-C17-H17A & 109.5 \\
\hline $\mathrm{C} 9-\mathrm{C} 8-\mathrm{Cl} 8$ & $118.90(17)$ & N14-C17-H17B & 109.5 \\
\hline $\mathrm{C} 7-\mathrm{C} 8-\mathrm{Cl} 8$ & $119.65(15)$ & $\mathrm{H} 17 \mathrm{~A}-\mathrm{C} 17-\mathrm{H} 17 \mathrm{~B}$ & 109.5 \\
\hline $\mathrm{C} 8-\mathrm{C} 9-\mathrm{C} 9 \mathrm{~A}$ & $120.55(19)$ & N14-C17-H17C & 109.5 \\
\hline $\mathrm{C} 8-\mathrm{C} 9-\mathrm{H} 9$ & 119.7 & $\mathrm{H} 17 \mathrm{~A}-\mathrm{C} 17-\mathrm{H} 17 \mathrm{C}$ & 109.5 \\
\hline $\mathrm{C} 9 \mathrm{~A}-\mathrm{C} 9-\mathrm{H} 9$ & 119.7 & $\mathrm{H} 17 \mathrm{~B}-\mathrm{C} 17-\mathrm{H} 17 \mathrm{C}$ & 109.5 \\
\hline C9-C9A-N10 & $117.09(17)$ & $\mathrm{O} 22-\mathrm{C} 21-\mathrm{O} 21$ & $121.8(2)$ \\
\hline $\mathrm{C} 9-\mathrm{C} 9 \mathrm{~A}-\mathrm{C} 5 \mathrm{~A}$ & $118.67(17)$ & $\mathrm{O} 22-\mathrm{C} 21-\mathrm{C} 22$ & $118.8(2)$ \\
\hline $\mathrm{N} 10-\mathrm{C} 9 \mathrm{~A}-\mathrm{C} 5 \mathrm{~A}$ & $124.00(17)$ & $\mathrm{O} 21-\mathrm{C} 21-\mathrm{C} 22$ & $119.42(19)$ \\
\hline $\mathrm{C} 11-\mathrm{N} 10-\mathrm{C} 9 \mathrm{~A}$ & $122.17(15)$ & $\mathrm{C} 21-\mathrm{O} 21-\mathrm{H} 21$ & $109(3)$ \\
\hline $\mathrm{N} 10-\mathrm{C} 11-\mathrm{N} 11$ & $118.36(16)$ & $\mathrm{C} 23-\mathrm{C} 22-\mathrm{C} 21$ & $131.1(2)$ \\
\hline $\mathrm{N} 10-\mathrm{C} 11-\mathrm{C} 11 \mathrm{~A}$ & $126.65(16)$ & $\mathrm{C} 23-\mathrm{C} 22-\mathrm{H} 22$ & 114.5 \\
\hline $\mathrm{N} 11-\mathrm{C} 11-\mathrm{C} 11 \mathrm{~A}$ & $114.80(15)$ & $\mathrm{C} 21-\mathrm{C} 22-\mathrm{H} 22$ & 114.5 \\
\hline $\mathrm{C} 4 \mathrm{~A}-\mathrm{C} 11 \mathrm{~A}-\mathrm{C} 1$ & $119.06(17)$ & $\mathrm{C} 22-\mathrm{C} 23-\mathrm{C} 24$ & $129.9(2)$ \\
\hline $\mathrm{C} 4 \mathrm{~A}-\mathrm{C} 11 \mathrm{~A}-\mathrm{C} 11$ & $120.61(16)$ & $\mathrm{C} 22-\mathrm{C} 23-\mathrm{H} 23$ & 115.1 \\
\hline $\mathrm{C} 1-\mathrm{C} 11 \mathrm{~A}-\mathrm{C} 11$ & $120.28(17)$ & $\mathrm{C} 24-\mathrm{C} 23-\mathrm{H} 23$ & 115.1 \\
\hline $\mathrm{C} 11-\mathrm{N} 11-\mathrm{C} 16$ & $122.04(15)$ & $\mathrm{O} 23-\mathrm{C} 24-\mathrm{O} 24$ & $122.78(19)$ \\
\hline $\mathrm{C} 11-\mathrm{N} 11-\mathrm{C} 12$ & $118.45(15)$ & $\mathrm{O} 23-\mathrm{C} 24-\mathrm{C} 23$ & $117.38(18)$ \\
\hline $\mathrm{C} 16-\mathrm{N} 11-\mathrm{C} 12$ & $111.09(14)$ & $\mathrm{O} 24-\mathrm{C} 24-\mathrm{C} 23$ & $119.84(19)$ \\
\hline $\mathrm{N} 11-\mathrm{C} 12-\mathrm{C} 13$ & $111.93(15)$ & $\mathrm{C} 24-\mathrm{O} 24-\mathrm{H} 21$ & $110.7(19)$ \\
\hline $\mathrm{N} 11-\mathrm{C} 12-\mathrm{H} 12 \mathrm{~A}$ & 109.2 & $\mathrm{H} 31 \mathrm{~A}-\mathrm{O} 31-\mathrm{H} 31 \mathrm{~B}$ & 113.2 \\
\hline $\mathrm{C} 13-\mathrm{C} 12-\mathrm{H} 12 \mathrm{~A}$ & 109.2 & & \\
\hline $\mathrm{C} 11 \mathrm{~A}-\mathrm{C} 1-\mathrm{C} 2-\mathrm{C} 3$ & $0.3(3)$ & $\mathrm{C} 4-\mathrm{C} 4 \mathrm{~A}-\mathrm{C} 11 \mathrm{~A}-\mathrm{C} 11$ & $171.79(17)$ \\
\hline $\mathrm{C} 1-\mathrm{C} 2-\mathrm{C} 3-\mathrm{C} 4$ & $-3.7(3)$ & $\mathrm{N} 5-\mathrm{C} 4 \mathrm{~A}-\mathrm{C} 11 \mathrm{~A}-\mathrm{C} 11$ & $-8.6(3)$ \\
\hline $\mathrm{C} 2-\mathrm{C} 3-\mathrm{C} 4-\mathrm{C} 4 \mathrm{~A}$ & $2.4(3)$ & $\mathrm{C} 2-\mathrm{C} 1-\mathrm{C} 11 \mathrm{~A}-\mathrm{C} 4 \mathrm{~A}$ & $4.3(3)$ \\
\hline $\mathrm{C} 3-\mathrm{C} 4-\mathrm{C} 4 \mathrm{~A}-\mathrm{C} 11 \mathrm{~A}$ & $2.3(3)$ & $\mathrm{C} 2-\mathrm{C} 1-\mathrm{C} 11 \mathrm{~A}-\mathrm{C} 11$ & $-173.05(19)$ \\
\hline $\mathrm{C} 3-\mathrm{C} 4-\mathrm{C} 4 \mathrm{~A}-\mathrm{N} 5$ & $-177.34(18)$ & $\mathrm{N} 10-\mathrm{C} 11-\mathrm{C} 11 \mathrm{~A}-\mathrm{C} 4 \mathrm{~A}$ & $45.2(3)$ \\
\hline $\mathrm{C} 11 \mathrm{~A}-\mathrm{C} 4 \mathrm{~A}-\mathrm{N} 5-\mathrm{C} 5 \mathrm{~A}$ & $-59.5(2)$ & $\mathrm{N} 11-\mathrm{C} 11-\mathrm{C} 11 \mathrm{~A}-\mathrm{C} 4 \mathrm{~A}$ & $-139.81(18)$ \\
\hline $\mathrm{C} 4-\mathrm{C} 4 \mathrm{~A}-\mathrm{N} 5-\mathrm{C} 5 \mathrm{~A}$ & $120.07(19)$ & $\mathrm{N} 10-\mathrm{C} 11-\mathrm{C} 11 \mathrm{~A}-\mathrm{C} 1$ & $-137.5(2)$ \\
\hline $\mathrm{C} 4 \mathrm{~A}-\mathrm{N} 5-\mathrm{C} 5 \mathrm{~A}-\mathrm{C} 6$ & $-116.17(19)$ & $\mathrm{N} 11-\mathrm{C} 11-\mathrm{C} 11 \mathrm{~A}-\mathrm{C} 1$ & $37.5(3)$ \\
\hline
\end{tabular}




$\begin{array}{llll}\mathrm{C} 4 \mathrm{~A}-\mathrm{N} 5-\mathrm{C} 5 \mathrm{~A}-\mathrm{C} 9 \mathrm{~A} & 64.0(2) & \mathrm{N} 10-\mathrm{C} 11-\mathrm{N} 11-\mathrm{C} 16 & -142.60(19) \\ \mathrm{C} 9 \mathrm{~A}-\mathrm{C} 5 \mathrm{~A}-\mathrm{C} 6-\mathrm{C} 7 & -0.3(3) & \mathrm{C} 11 \mathrm{~A}-\mathrm{C} 11-\mathrm{N} 11-\mathrm{C} 16 & 42.0(2) \\ \mathrm{N} 5-\mathrm{C} 5 \mathrm{~A}-\mathrm{C} 6-\mathrm{C} 7 & 179.87(17) & \mathrm{N} 10-\mathrm{C} 11-\mathrm{N} 11-\mathrm{C} 12 & 2.7(3) \\ \mathrm{C} 5 \mathrm{~A}-\mathrm{C} 6-\mathrm{C} 7-\mathrm{C} 8 & -0.4(3) & \mathrm{C} 11 \mathrm{~A}-\mathrm{C} 11-\mathrm{N} 11-\mathrm{C} 12 & -172.74(16) \\ \mathrm{C} 6-\mathrm{C} 7-\mathrm{C} 8-\mathrm{C} 9 & 1.6(3) & \mathrm{C} 11-\mathrm{N} 11-\mathrm{C} 12-\mathrm{C} 13 & -91.0(2) \\ \mathrm{C} 6-\mathrm{C} 7-\mathrm{C} 8-\mathrm{C} 18 & -179.85(15) & \mathrm{C} 16-\mathrm{N} 11-\mathrm{C} 12-\mathrm{C} 13 & 57.8(2) \\ \mathrm{C} 7-\mathrm{C} 8-\mathrm{C} 9-\mathrm{C} 9 \mathrm{~A} & -2.0(3) & \mathrm{N} 11-\mathrm{C} 12-\mathrm{C} 13-\mathrm{N} 14 & -57.45(19) \\ \mathrm{C} 18-\mathrm{C} 8-\mathrm{C} 9-\mathrm{C} 9 \mathrm{~A} & 179.39(14) & \mathrm{C} 12-\mathrm{C} 13-\mathrm{N} 14-\mathrm{C} 17 & -178.53(16) \\ \mathrm{C} 8-\mathrm{C} 9-\mathrm{C} 9 \mathrm{~A}-\mathrm{N} 10 & 175.80(17) & \mathrm{C} 12-\mathrm{C} 13-\mathrm{N} 14-\mathrm{C} 15 & 55.9(2) \\ \mathrm{C} 8-\mathrm{C} 9-\mathrm{C} 9 \mathrm{~A}-\mathrm{C} 5 \mathrm{~A} & 1.2(3) & \mathrm{C} 17-\mathrm{N} 14-\mathrm{C} 15-\mathrm{C} 16 & 179.67(18) \\ \mathrm{C} 6-\mathrm{C} 5 \mathrm{~A}-\mathrm{C} 9 \mathrm{~A}-\mathrm{C} 9 & -0.1(3) & \mathrm{C} 13-\mathrm{N} 14-\mathrm{C} 15-\mathrm{C} 16 & -55.1(2) \\ \mathrm{N} 5-\mathrm{C} 5 \mathrm{~A}-\mathrm{C} 9 \mathrm{~A}-\mathrm{C} 9 & 179.71(16) & \mathrm{C} 11-\mathrm{N} 11-\mathrm{C} 16-\mathrm{C} 15 & 91.5(2) \\ \mathrm{C} 6-\mathrm{C} 5 \mathrm{~A}-\mathrm{C} 9 \mathrm{~A}-\mathrm{N} 10 & -174.23(16) & \mathrm{C} 12-\mathrm{N} 11-\mathrm{C} 16-\mathrm{C} 15 & -56.0(2) \\ \mathrm{N} 5-\mathrm{C} 5 \mathrm{~A}-\mathrm{C} 9 \mathrm{~A}-\mathrm{N} 10 & 5.6(3) & \mathrm{N} 14-\mathrm{C} 15-\mathrm{C} 16-\mathrm{N} 11 & 54.9(2) \\ \mathrm{C} 9-\mathrm{C} 9 \mathrm{~A}-\mathrm{N} 10-\mathrm{C} 11 & 141.11(19) & \mathrm{O} 22-\mathrm{C} 21-\mathrm{C} 22-\mathrm{C} 23 & 171.5(3) \\ \mathrm{C} 5 \mathrm{~A}-\mathrm{C} 9 \mathrm{~A}-\mathrm{N} 10-\mathrm{C} 11 & -44.6(3) & \mathrm{O} 21-\mathrm{C} 21-\mathrm{C} 22-\mathrm{C} 23 & -8.7(5) \\ \text { C9A-N10-C11-N11 } & -173.90(17) & \mathrm{C} 21-\mathrm{C} 22-\mathrm{C} 23-\mathrm{C} 24 & 1.3(5) \\ \text { C9A-N10-C11-C11A } & 0.9(3) & \mathrm{C} 22-\mathrm{C} 23-\mathrm{C} 24-\mathrm{O} 23 & -174.8(3) \\ \text { C4-C4A-C11A-C1 } & -5.6(3) & \mathrm{C} 22-\mathrm{C} 23-\mathrm{C} 24-\mathrm{O} 24 & 6.0(5) \\ \text { N5-C4A-C11A-C1 } & 174.05(17) & & \end{array}$

Hydrogen-bond geometry $\left(A,{ }^{\circ}\right)$

$\mathrm{Cg} 1$ and $\mathrm{Cg} 2$ represent the centroids of the rings $(\mathrm{C} 5 \mathrm{~A}, \mathrm{C} 6-\mathrm{C} 9, \mathrm{C} 9 \mathrm{~A})$ and $(\mathrm{C} 1-\mathrm{C} 4, \mathrm{C} 4 \mathrm{~A}, \mathrm{C} 11 \mathrm{~A})$, respectively.

\begin{tabular}{lllll}
\hline$D-\mathrm{H} \cdots A$ & $D-\mathrm{H}$ & $\mathrm{H} \cdots A$ & $D \cdots A$ & $D-\mathrm{H} \cdots A$ \\
\hline $\mathrm{N} 5-\mathrm{H} 5 \cdots \mathrm{O} 22^{\mathrm{i}}$ & $0.86(3)$ & $2.24(3)$ & $3.084(2)$ & $170(3)$ \\
$\mathrm{N} 14-\mathrm{H} 14 \cdots \mathrm{O} 23$ & $0.87(3)$ & $1.82(3)$ & $2.688(2)$ & $173(2)$ \\
$\mathrm{O} 21-\mathrm{H} 21 \cdots \mathrm{O} 24$ & $1.00(5)$ & $1.46(5)$ & $2.420(3)$ & $157(5)$ \\
$\mathrm{O} 31-\mathrm{H} 31 A \cdots \mathrm{O} 24$ & 0.90 & 2.05 & $2.913(11)$ & 160 \\
$\mathrm{O} 31-\mathrm{H} 31 B \cdots \mathrm{O} 21^{\mathrm{ii}}$ & 0.90 & 2.37 & $3.232(11)$ & 161 \\
$\mathrm{C} 12-\mathrm{H} 12 A \cdots \mathrm{O} 22^{\text {iii }}$ & 0.99 & 2.48 & $3.308(2)$ & 141 \\
$\mathrm{C} 15-\mathrm{H} 15 A \cdots C g 1^{\text {iv }}$ & 0.99 & 2.95 & $3.642(2)$ & 128 \\
$\mathrm{C} 15-\mathrm{H} 15 B \cdots C g 2^{\text {iv }}$ & 0.99 & 2.95 & $3.759(2)$ & 139
\end{tabular}

Symmetry codes: (i) $x-1,-y+3 / 2, z-1 / 2$; (ii) $-x+2,-y+2,-z+1$; (iii) $-x+2,-y+1,-z+1$; (iv) $-x+1, y+1 / 2,-z+1 / 2$.

(III) Clozapinium 2-hydroxybenzoate

Crystal data

$\mathrm{C}_{18} \mathrm{H}_{20} \mathrm{ClN}_{4}^{+} \cdot \mathrm{C}_{7} \mathrm{H}_{5} \mathrm{O}_{3}^{-}$

$M_{r}=464.94$

Monoclinic, $C c$

$a=17.4296(5) \AA$

$b=15.3728(5) \AA$

$c=8.6359(3) \AA$

$\beta=90.325(3)^{\circ}$

$V=2313.88(13) \AA^{3}$

$Z=4$
$F(000)=976$

$D_{\mathrm{x}}=1.335 \mathrm{Mg} \mathrm{m}^{-3}$

$\mathrm{Cu} K \alpha$ radiation, $\lambda=1.54184 \AA$

Cell parameters from 4069 reflections

$\theta=3.8-72.6^{\circ}$

$\mu=1.75 \mathrm{~mm}^{-1}$

$T=173 \mathrm{~K}$

Block, colourless

$0.42 \times 0.36 \times 0.20 \mathrm{~mm}$ 


\section{Data collection}

Agilent Eos Gemini diffractometer

Radiation source: Enhance $(\mathrm{Cu}) \mathrm{X}$-ray Source $\omega$ scans

Absorption correction: multi-scan

(CrysAlis RED; Agilent, 2012)

$T_{\min }=0.399, T_{\max }=0.705$

7244 measured reflections

\section{Refinement}

Refinement on $F^{2}$

Least-squares matrix: full

$R\left[F^{2}>2 \sigma\left(F^{2}\right)\right]=0.056$

$w R\left(F^{2}\right)=0.141$

$S=1.08$

4069 reflections

308 parameters

2 restraints

Hydrogen site location: difference Fourier map
4069 independent reflections

3962 reflections with $I>2 \sigma(I)$

$R_{\text {int }}=0.048$

$\theta_{\text {max }}=72.6^{\circ}, \theta_{\min }=3.8^{\circ}$

$h=-21 \rightarrow 21$

$k=-10 \rightarrow 18$

$l=-10 \rightarrow 10$
$\mathrm{H}$ atoms treated by a mixture of independent and constrained refinement

$w=1 /\left[\sigma^{2}\left(F_{\mathrm{o}}^{2}\right)+(0.1069 P)^{2}\right]$ where $P=\left(F_{\mathrm{o}}^{2}+2 F_{\mathrm{c}}^{2}\right) / 3$

$(\Delta / \sigma)_{\max }<0.001$

$\Delta \rho_{\max }=0.53$ e $\AA^{-3}$

$\Delta \rho_{\min }=-0.36$ e $\AA^{-3}$

Absolute structure: Flack $x$ determined using 1674 quotients $\left[\left(I^{+}\right)-\left(I^{-}\right)\right] /\left[\left(I^{+}\right)+\left(I^{\prime}\right)\right]$ (Parsons et al., 2013)

Absolute structure parameter: -0.022 (17)

Special details

Geometry. All e.s.d.'s (except the e.s.d. in the dihedral angle between two 1.s. planes) are estimated using the full covariance matrix. The cell e.s.d.'s are taken into account individually in the estimation of e.s.d.'s in distances, angles and torsion angles; correlations between e.s.d.'s in cell parameters are only used when they are defined by crystal symmetry. An approximate (isotropic) treatment of cell e.s.d.'s is used for estimating e.s.d.'s involving 1.s. planes.

Fractional atomic coordinates and isotropic or equivalent isotropic displacement parameters $\left(\hat{A}^{2}\right)$

\begin{tabular}{lllll}
\hline & $x$ & $y$ & $z$ & $U_{\text {iso }} * / U_{\text {eq }}$ \\
\hline C1 & $0.68573(19)$ & $0.4925(2)$ & $0.8347(4)$ & $0.0296(6)$ \\
H1 & 0.6566 & 0.5324 & 0.8945 & $0.036^{*}$ \\
C2 & $0.7650(2)$ & $0.4966(2)$ & $0.8402(4)$ & $0.0346(7)$ \\
H2 & 0.7899 & 0.5394 & 0.9018 & $0.042^{*}$ \\
C3 & $0.80783(19)$ & $0.4372(2)$ & $0.7545(4)$ & $0.0338(7)$ \\
H3 & 0.8623 & 0.4405 & 0.7552 & $0.041^{*}$ \\
C4 & $0.77094(19)$ & $0.3730(2)$ & $0.6681(4)$ & $0.0322(7)$ \\
H4 & 0.8003 & 0.3310 & 0.6136 & $0.039^{*}$ \\
C4A & $0.69150(18)$ & $0.3700(2)$ & $0.6611(3)$ & $0.0273(6)$ \\
N5 & $0.65422(16)$ & $0.30589(18)$ & $0.5690(3)$ & $0.0306(6)$ \\
H5 & $0.688(3)$ & $0.276(3)$ & $0.511(6)$ & $0.037^{*}$ \\
C5A & $0.60999(17)$ & $0.2452(2)$ & $0.6565(3)$ & $0.0290(6)$ \\
C6 & $0.6279(2)$ & $0.1568(2)$ & $0.6588(4)$ & $0.0368(7)$ \\
H6 & 0.6702 & 0.1363 & 0.6004 & $0.044 *$ \\
C7 & $0.5850(2)$ & $0.0983(2)$ & $0.7448(5)$ & $0.0403(8)$ \\
H7 & 0.5969 & 0.0380 & 0.7442 & $0.048^{*}$ \\
C8 & $0.5249(2)$ & $0.1300(2)$ & $0.8312(5)$ & $0.0369(7)$ \\
C18 & $0.47247(6)$ & $0.05843(6)$ & $0.94789(14)$ & $0.0561(3)$ \\
C9 & $0.50474(19)$ & $0.2173(2)$ & $0.8300(4)$ & $0.0332(7)$
\end{tabular}




$\begin{array}{lllll}\text { H9 } & 0.4622 & 0.2369 & 0.8886 & 0.040^{*} \\ \text { C9A } & 0.54720(18) & 0.2761(2) & 0.7424(4) & 0.0287(6) \\ \text { N10 } & 0.51934(16) & 0.36170(18) & 0.7350(3) & 0.0294(5) \\ \text { C11 } & 0.56224(18) & 0.4295(2) & 0.7362(3) & 0.0267(6) \\ \text { C11A } & 0.64754(19) & 0.4310(2) & 0.7428(3) & 0.0272(6) \\ \text { N11 } & 0.52619(16) & 0.50963(18) & 0.7496(3) & 0.0303(6) \\ \text { C12 } & 0.44338(19) & 0.5111(2) & 0.7757(4) & 0.0334(7) \\ \text { H12A } & 0.4281 & 0.4591 & 0.8356 & 0.040^{*} \\ \text { H12B } & 0.4159 & 0.5098 & 0.6751 & 0.040^{*} \\ \text { C13 } & 0.4218(2) & 0.5929(2) & 0.8641(4) & 0.0357(7) \\ \text { H13A } & 0.3654 & 0.5949 & 0.8774 & 0.043^{*} \\ \text { H13B } & 0.4458 & 0.5914 & 0.9682 & 0.043^{*} \\ \text { N14 } & 0.44753(17) & 0.6726(2) & 0.7804(3) & 0.0331(6) \\ \text { H14 } & 0.423(3) & 0.675(3) & 0.690(7) & 0.040^{*} \\ \text { C15 } & 0.5313(2) & 0.6687(2) & 0.7446(4) & 0.0338(7) \\ \text { H15A } & 0.5614 & 0.6712 & 0.8422 & 0.041^{*} \\ \text { H15B } & 0.5457 & 0.7195 & 0.6805 & 0.041^{*} \\ \text { C16 } & 0.55040(19) & 0.5851(2) & 0.6580(4) & 0.0306(6) \\ \text { H16A } & 0.5237 & 0.5847 & 0.5565 & 0.037^{*} \\ \text { H16B } & 0.6063 & 0.5821 & 0.6393 & 0.037^{*} \\ \text { C17 } & 0.4297(3) & 0.7522(3) & 0.8713(5) & 0.0482(9) \\ \text { H17A } & 0.4588 & 0.7513 & 0.9687 & 0.072^{*} \\ \text { H17B } & 0.3747 & 0.7539 & 0.8937 & 0.072^{*} \\ \text { H17C } & 0.4439 & 0.8038 & 0.8114 & 0.072^{*} \\ \text { C21 } & 0.28195(18) & 0.6783(2) & 0.3237(4) & 0.0306(6) \\ \text { C22 } & 0.2327(2) & 0.6192(3) & 0.2489(5) & 0.0462(9) \\ \text { C23 } & 0.1947(3) & 0.6426(4) & 0.1142(7) & 0.0657(14) \\ \text { H23A } & 0.1623 & 0.6020 & 0.0627 & 0.079^{*} \\ \text { C24 } & 0.2041(3) & 0.7258(5) & 0.0548(6) & 0.0673(15) \\ \text { H24 } & 0.1774 & 0.7422 & -0.0369 & 0.081^{*} \\ \text { C25 } & 0.2518(3) & 0.7851(3) & 0.1272(5) & 0.0509(10) \\ \text { H25 } & 0.2576 & 0.8419 & 0.0856 & 0.061^{*} \\ \text { C26 } & 0.2911(2) & 0.7617(2) & 0.2602(4) & 0.0354(7) \\ \text { H26 } & 0.3245 & 0.8022 & 0.3089 & 0.042^{*} \\ \text { C27 } & 0.32403(17) & 0.6517(2) & 0.4680(4) & 0.0286(6) \\ \text { O21 } & 0.31483(16) & 0.57583(18) & 0.5174(4) & 0.0445(6) \\ \text { O22 } & 0.36650(14) & 0.70751(16) & 0.5327(3) & 0.0332(5) \\ \text { O23 } & 0.2212(2) & 0.5383(2) & 0.3064(5) & 0.0661(10) \\ \text { H23 } & 0.259(6) & 0.544(6) & 0.394(13) & 0.099^{*} \\ & & & & \end{array}$

Atomic displacement parameters $\left(\AA^{2}\right)$

\begin{tabular}{lllllll}
\hline & $U^{11}$ & $U^{22}$ & $U^{33}$ & $U^{12}$ & $U^{13}$ & $U^{23}$ \\
\hline C1 & $0.0314(15)$ & $0.0289(13)$ & $0.0285(14)$ & $0.0049(11)$ & $0.0008(12)$ & $-0.0026(11)$ \\
C2 & $0.0345(17)$ & $0.0331(15)$ & $0.0363(16)$ & $-0.0012(12)$ & $-0.0036(13)$ & $-0.0015(12)$ \\
C3 & $0.0239(13)$ & $0.0341(15)$ & $0.0433(18)$ & $0.0016(11)$ & $-0.0013(12)$ & $0.0033(12)$ \\
C4 & $0.0311(16)$ & $0.0340(15)$ & $0.0317(16)$ & $0.0088(12)$ & $0.0028(12)$ & $0.0018(12)$ \\
C4A & $0.0305(16)$ & $0.0285(14)$ & $0.0229(13)$ & $0.0044(11)$ & $0.0013(11)$ & $0.0035(10)$
\end{tabular}




\begin{tabular}{|c|c|c|c|c|c|c|}
\hline N5 & $0.0311(13)$ & $0.0348(13)$ & $0.0259(12)$ & $0.0058(11)$ & $0.0038(10)$ & $-0.0053(10)$ \\
\hline $\mathrm{C} 5 \mathrm{~A}$ & $0.0255(15)$ & $0.0335(15)$ & $0.0279(15)$ & $0.0030(11)$ & $-0.0050(12)$ & $-0.0046(11)$ \\
\hline C6 & $0.0329(15)$ & $0.0346(17)$ & $0.0429(19)$ & $0.0062(12)$ & $-0.0030(13)$ & $-0.0084(13)$ \\
\hline $\mathrm{C} 7$ & $0.0354(17)$ & $0.0276(15)$ & $0.058(2)$ & $0.0028(12)$ & $-0.0092(15)$ & $-0.0047(13)$ \\
\hline $\mathrm{C} 8$ & $0.0300(15)$ & $0.0340(16)$ & $0.0466(19)$ & $-0.0048(12)$ & $-0.0060(14)$ & $0.0011(13)$ \\
\hline $\mathrm{Cl} 8$ & $0.0461(5)$ & $0.0410(5)$ & $0.0813(8)$ & -0.0089 & $0.0056(5)$ & $0.0139(4)$ \\
\hline C9 & $0.0243(14)$ & $0.0387(16)$ & $0.0364(17)$ & $-0.0001(11)$ & $-0.0050(12)$ & $-0.0021(12)$ \\
\hline C9A & $0.0241(13)$ & $0.0336(15)$ & $0.0281(14)$ & $0.0035(11)$ & $-0.0074(11)$ & $-0.0035(11)$ \\
\hline N10 & $0.0253(11)$ & $0.0345(13)$ & $0.0284(13)$ & $0.0068(10)$ & $-0.0013(10)$ & $0.0003(10)$ \\
\hline C11 & $0.0272(15)$ & $0.0338(15)$ & $0.0190(13)$ & $0.0063(11)$ & $0.0000(10)$ & $0.0004(10)$ \\
\hline $\mathrm{C} 11 \mathrm{~A}$ & $0.0277(15)$ & $0.0296(13)$ & $0.0242(14)$ & $0.0034(11)$ & $0.0004(11)$ & $0.0022(10)$ \\
\hline N11 & $0.0272(13)$ & $0.0328(13)$ & $0.0310(13)$ & $0.0088(10)$ & $0.0028(10)$ & $0.0038(10)$ \\
\hline $\mathrm{C} 12$ & $0.0269(15)$ & $0.0355(16)$ & $0.0378(17)$ & $0.0087(12)$ & $0.0056(12)$ & $0.0048(12)$ \\
\hline $\mathrm{C} 13$ & $0.0338(16)$ & $0.0443(18)$ & $0.0290(15)$ & $0.0149(14)$ & $0.0048(12)$ & $0.0022(13)$ \\
\hline N14 & $0.0352(14)$ & $0.0367(14)$ & $0.0271(14)$ & $0.0137(11)$ & $-0.0073(11)$ & $-0.0033(10)$ \\
\hline $\mathrm{C} 15$ & $0.0329(15)$ & $0.0329(15)$ & $0.0357(16)$ & $0.0062(12)$ & $-0.0082(13)$ & $0.0000(12)$ \\
\hline $\mathrm{C} 16$ & $0.0315(15)$ & $0.0311(15)$ & $0.0292(14)$ & $0.0069(11)$ & $0.0017(12)$ & $0.0038(12)$ \\
\hline $\mathrm{C} 17$ & $0.053(2)$ & $0.049(2)$ & $0.0425(19)$ & $0.0227(18)$ & $-0.0116(17)$ & $-0.0150(16)$ \\
\hline $\mathrm{C} 21$ & $0.0250(13)$ & $0.0386(16)$ & $0.0282(15)$ & $0.0046(12)$ & $-0.0008(11)$ & $-0.0034(12)$ \\
\hline $\mathrm{C} 22$ & $0.0366(18)$ & $0.053(2)$ & $0.049(2)$ & $-0.0049(16)$ & $-0.0060(16)$ & $-0.0092(17)$ \\
\hline $\mathrm{C} 23$ & $0.053(3)$ & $0.093(4)$ & $0.051(3)$ & $-0.011(3)$ & $-0.021(2)$ & $-0.014(3)$ \\
\hline $\mathrm{C} 24$ & $0.052(3)$ & $0.114(5)$ & $0.036(2)$ & $0.012(3)$ & $-0.0204(19)$ & $0.002(2)$ \\
\hline $\mathrm{C} 25$ & $0.047(2)$ & $0.068(3)$ & $0.0374(19)$ & $0.0157(19)$ & $-0.0009(17)$ & $0.0149(18)$ \\
\hline $\mathrm{C} 26$ & $0.0311(16)$ & $0.0442(18)$ & $0.0307(16)$ & $0.0052(13)$ & $-0.0020(13)$ & $0.0025(13)$ \\
\hline $\mathrm{C} 27$ & $0.0212(13)$ & $0.0339(15)$ & $0.0308(15)$ & $0.0054(11)$ & $0.0030(11)$ & $-0.0010(11)$ \\
\hline $\mathrm{O} 21$ & $0.0373(14)$ & $0.0400(13)$ & $0.0562(16)$ & $-0.0004(11)$ & $-0.0038(11)$ & $0.0128(12)$ \\
\hline $\mathrm{O} 22$ & $0.0315(11)$ & $0.0375(11)$ & $0.0304(11)$ & $0.0034(9)$ & $-0.0075(9)$ & $-0.0002(8)$ \\
\hline $\mathrm{O} 23$ & $0.060(2)$ & $0.0520(17)$ & $0.086(3)$ & $-0.0213(17)$ & $-0.0142(18)$ & $-0.0066(18)$ \\
\hline
\end{tabular}

Geometric parameters ( $\left.\AA,{ }^{\circ}\right)$

\begin{tabular}{llll}
\hline $\mathrm{C} 1-\mathrm{C} 2$ & $1.383(5)$ & $\mathrm{C} 12-\mathrm{H} 12 \mathrm{~B}$ & 0.9900 \\
$\mathrm{C} 1-\mathrm{C} 11 \mathrm{~A}$ & $1.400(5)$ & $\mathrm{C} 13-\mathrm{N} 14$ & $1.493(5)$ \\
$\mathrm{C} 1-\mathrm{H} 1$ & 0.9500 & $\mathrm{C} 13-\mathrm{H} 13 \mathrm{~A}$ & 0.9900 \\
$\mathrm{C} 2-\mathrm{C} 3$ & $1.395(5)$ & $\mathrm{C} 13-\mathrm{H} 13 \mathrm{~B}$ & $1.487(4)$ \\
$\mathrm{C} 2-\mathrm{H} 2$ & 0.9500 & $\mathrm{~N} 14-\mathrm{C} 17$ & $1.496(4)$ \\
$\mathrm{C} 3-\mathrm{C} 4$ & $1.393(5)$ & $\mathrm{N} 14-\mathrm{C} 15$ & $0.88(6)$ \\
$\mathrm{C} 3-\mathrm{H} 3$ & 0.9500 & $\mathrm{~N} 14-\mathrm{H} 14$ & $1.524(4)$ \\
$\mathrm{C} 4-\mathrm{C} 4 \mathrm{~A}$ & $1.386(4)$ & $\mathrm{C} 15-\mathrm{C} 16$ & 0.9900 \\
$\mathrm{C} 4-\mathrm{H} 4$ & 0.9500 & $\mathrm{C} 15-\mathrm{H} 15 \mathrm{~A}$ & 0.9900 \\
$\mathrm{C} 4 \mathrm{~A}-\mathrm{C} 11 \mathrm{~A}$ & $1.404(4)$ & $\mathrm{C} 15-\mathrm{H} 15 \mathrm{~B}$ & 0.9900 \\
$\mathrm{C} 4 \mathrm{~A}-\mathrm{N} 5$ & $1.422(4)$ & $\mathrm{C} 16-\mathrm{H} 16 \mathrm{~A}$ & 0.9900 \\
$\mathrm{~N} 5-\mathrm{C} 5 \mathrm{~A}$ & $1.429(4)$ & $\mathrm{C} 16-\mathrm{H} 16 \mathrm{~B}$ & 0.9800 \\
$\mathrm{~N} 5-\mathrm{H} 5$ & $0.90(5)$ & $\mathrm{C} 17-\mathrm{H} 17 \mathrm{~A}$ & 0.9800 \\
$\mathrm{C} 5 \mathrm{~A}-\mathrm{C} 6$ & $1.394(5)$ & $\mathrm{C} 17-\mathrm{H} 17 \mathrm{~B}$ & 0.9800 \\
$\mathrm{C} 5 \mathrm{~A}-\mathrm{C} 9 \mathrm{~A}$ & $1.408(4)$ & $\mathrm{C} 17-\mathrm{H} 17 \mathrm{C}$ & $1.403(5)$ \\
$\mathrm{C} 6-\mathrm{C} 7$ & $1.387(6)$ & $\mathrm{C} 21-\mathrm{C} 26$ & $1.406(5)$ \\
$\mathrm{C} 6-\mathrm{H} 6$ & 0.9500 & $\mathrm{C} 21-\mathrm{C} 22$ &
\end{tabular}




\begin{tabular}{|c|c|c|c|}
\hline $\mathrm{C} 7-\mathrm{C} 8$ & $1.379(6)$ & $\mathrm{C} 21-\mathrm{C} 27$ & $1.499(4)$ \\
\hline $\mathrm{C} 7-\mathrm{H} 7$ & 0.9500 & $\mathrm{C} 22-\mathrm{O} 23$ & $1.355(6)$ \\
\hline $\mathrm{C} 8-\mathrm{C} 9$ & $1.387(5)$ & $\mathrm{C} 22-\mathrm{C} 23$ & $1.383(7)$ \\
\hline $\mathrm{C} 8-\mathrm{Cl} 8$ & $1.752(4)$ & $\mathrm{C} 23-\mathrm{C} 24$ & $1.389(9)$ \\
\hline $\mathrm{C} 9-\mathrm{C} 9 \mathrm{~A}$ & $1.394(5)$ & $\mathrm{C} 23-\mathrm{H} 23 \mathrm{~A}$ & 0.9500 \\
\hline $\mathrm{C} 9-\mathrm{H} 9$ & 0.9500 & $\mathrm{C} 24-\mathrm{C} 25$ & $1.381(9)$ \\
\hline $\mathrm{C} 9 \mathrm{~A}-\mathrm{N} 10$ & $1.405(4)$ & $\mathrm{C} 24-\mathrm{H} 24$ & 0.9500 \\
\hline $\mathrm{N} 10-\mathrm{C} 11$ & $1.283(5)$ & $\mathrm{C} 25-\mathrm{C} 26$ & $1.381(5)$ \\
\hline $\mathrm{C} 11-\mathrm{N} 11$ & $1.388(4)$ & $\mathrm{C} 25-\mathrm{H} 25$ & 0.9500 \\
\hline $\mathrm{C} 11-\mathrm{C} 11 \mathrm{~A}$ & $1.488(4)$ & $\mathrm{C} 26-\mathrm{H} 26$ & 0.9500 \\
\hline $\mathrm{N} 11-\mathrm{C} 12$ & $1.462(4)$ & $\mathrm{C} 27-\mathrm{O} 21$ & $1.253(4)$ \\
\hline $\mathrm{N} 11-\mathrm{C} 16$ & $1.467(4)$ & $\mathrm{C} 27-\mathrm{O} 22$ & $1.261(4)$ \\
\hline $\mathrm{C} 12-\mathrm{C} 13$ & $1.520(4)$ & $\mathrm{O} 23-\mathrm{H} 23$ & $1.00(11)$ \\
\hline $\mathrm{C} 12-\mathrm{H} 12 \mathrm{~A}$ & 0.9900 & & \\
\hline $\mathrm{C} 2-\mathrm{C} 1-\mathrm{C} 11 \mathrm{~A}$ & $121.4(3)$ & $\mathrm{N} 14-\mathrm{C} 13-\mathrm{C} 12$ & $111.1(3)$ \\
\hline $\mathrm{C} 2-\mathrm{C} 1-\mathrm{H} 1$ & 119.3 & $\mathrm{~N} 14-\mathrm{C} 13-\mathrm{H} 13 \mathrm{~A}$ & 109.4 \\
\hline $\mathrm{C} 11 \mathrm{~A}-\mathrm{C} 1-\mathrm{H} 1$ & 119.3 & $\mathrm{C} 12-\mathrm{C} 13-\mathrm{H} 13 \mathrm{~A}$ & 109.4 \\
\hline $\mathrm{C} 1-\mathrm{C} 2-\mathrm{C} 3$ & $119.3(3)$ & $\mathrm{N} 14-\mathrm{C} 13-\mathrm{H} 13 \mathrm{~B}$ & 109.4 \\
\hline $\mathrm{C} 1-\mathrm{C} 2-\mathrm{H} 2$ & 120.3 & $\mathrm{C} 12-\mathrm{C} 13-\mathrm{H} 13 \mathrm{~B}$ & 109.4 \\
\hline $\mathrm{C} 3-\mathrm{C} 2-\mathrm{H} 2$ & 120.3 & $\mathrm{H} 13 \mathrm{~A}-\mathrm{C} 13-\mathrm{H} 13 \mathrm{~B}$ & 108.0 \\
\hline $\mathrm{C} 4-\mathrm{C} 3-\mathrm{C} 2$ & $120.1(3)$ & $\mathrm{C} 17-\mathrm{N} 14-\mathrm{C} 13$ & $110.8(3)$ \\
\hline $\mathrm{C} 4-\mathrm{C} 3-\mathrm{H} 3$ & 120.0 & $\mathrm{C} 17-\mathrm{N} 14-\mathrm{C} 15$ & $110.4(3)$ \\
\hline $\mathrm{C} 2-\mathrm{C} 3-\mathrm{H} 3$ & 120.0 & $\mathrm{C} 13-\mathrm{N} 14-\mathrm{C} 15$ & $111.3(3)$ \\
\hline $\mathrm{C} 4 \mathrm{~A}-\mathrm{C} 4-\mathrm{C} 3$ & $120.3(3)$ & $\mathrm{C} 17-\mathrm{N} 14-\mathrm{H} 14$ & $110(3)$ \\
\hline $\mathrm{C} 4 \mathrm{~A}-\mathrm{C} 4-\mathrm{H} 4$ & 119.9 & $\mathrm{C} 13-\mathrm{N} 14-\mathrm{H} 14$ & $108(3)$ \\
\hline $\mathrm{C} 3-\mathrm{C} 4-\mathrm{H} 4$ & 119.9 & $\mathrm{C} 15-\mathrm{N} 14-\mathrm{H} 14$ & $106(3)$ \\
\hline $\mathrm{C} 4-\mathrm{C} 4 \mathrm{~A}-\mathrm{C} 11 \mathrm{~A}$ & $120.3(3)$ & $\mathrm{N} 14-\mathrm{C} 15-\mathrm{C} 16$ & $110.5(3)$ \\
\hline $\mathrm{C} 4-\mathrm{C} 4 \mathrm{~A}-\mathrm{N} 5$ & $120.0(3)$ & $\mathrm{N} 14-\mathrm{C} 15-\mathrm{H} 15 \mathrm{~A}$ & 109.5 \\
\hline $\mathrm{C} 11 \mathrm{~A}-\mathrm{C} 4 \mathrm{~A}-\mathrm{N} 5$ & $119.7(3)$ & $\mathrm{C} 16-\mathrm{C} 15-\mathrm{H} 15 \mathrm{~A}$ & 109.5 \\
\hline $\mathrm{C} 4 \mathrm{~A}-\mathrm{N} 5-\mathrm{C} 5 \mathrm{~A}$ & $113.8(2)$ & $\mathrm{N} 14-\mathrm{C} 15-\mathrm{H} 15 \mathrm{~B}$ & 109.5 \\
\hline $\mathrm{C} 4 \mathrm{~A}-\mathrm{N} 5-\mathrm{H} 5$ & $111(3)$ & $\mathrm{C} 16-\mathrm{C} 15-\mathrm{H} 15 \mathrm{~B}$ & 109.5 \\
\hline $\mathrm{C} 5 \mathrm{~A}-\mathrm{N} 5-\mathrm{H} 5$ & $108(3)$ & $\mathrm{H} 15 \mathrm{~A}-\mathrm{C} 15-\mathrm{H} 15 \mathrm{~B}$ & 108.1 \\
\hline $\mathrm{C} 6-\mathrm{C} 5 \mathrm{~A}-\mathrm{C} 9 \mathrm{~A}$ & $119.7(3)$ & $\mathrm{N} 11-\mathrm{C} 16-\mathrm{C} 15$ & $109.8(3)$ \\
\hline $\mathrm{C} 6-\mathrm{C} 5 \mathrm{~A}-\mathrm{N} 5$ & $121.5(3)$ & $\mathrm{N} 11-\mathrm{C} 16-\mathrm{H} 16 \mathrm{~A}$ & 109.7 \\
\hline $\mathrm{C} 9 \mathrm{~A}-\mathrm{C} 5 \mathrm{~A}-\mathrm{N} 5$ & $118.8(3)$ & $\mathrm{C} 15-\mathrm{C} 16-\mathrm{H} 16 \mathrm{~A}$ & 109.7 \\
\hline $\mathrm{C} 7-\mathrm{C} 6-\mathrm{C} 5 \mathrm{~A}$ & $121.3(3)$ & $\mathrm{N} 11-\mathrm{C} 16-\mathrm{H} 16 \mathrm{~B}$ & 109.7 \\
\hline $\mathrm{C} 7-\mathrm{C} 6-\mathrm{H} 6$ & 119.4 & $\mathrm{C} 15-\mathrm{C} 16-\mathrm{H} 16 \mathrm{~B}$ & 109.7 \\
\hline $\mathrm{C} 5 \mathrm{~A}-\mathrm{C} 6-\mathrm{H} 6$ & 119.4 & $\mathrm{H} 16 \mathrm{~A}-\mathrm{C} 16-\mathrm{H} 16 \mathrm{~B}$ & 108.2 \\
\hline $\mathrm{C} 8-\mathrm{C} 7-\mathrm{C} 6$ & $118.3(3)$ & N14-C17-H17A & 109.5 \\
\hline $\mathrm{C} 8-\mathrm{C} 7-\mathrm{H} 7$ & 120.9 & N14-C17-H17B & 109.5 \\
\hline $\mathrm{C} 6-\mathrm{C} 7-\mathrm{H} 7$ & 120.9 & $\mathrm{H} 17 \mathrm{~A}-\mathrm{C} 17-\mathrm{H} 17 \mathrm{~B}$ & 109.5 \\
\hline $\mathrm{C} 7-\mathrm{C} 8-\mathrm{C} 9$ & $122.1(3)$ & $\mathrm{N} 14-\mathrm{C} 17-\mathrm{H} 17 \mathrm{C}$ & 109.5 \\
\hline $\mathrm{C} 7-\mathrm{C} 8-\mathrm{Cl} 8$ & $119.3(3)$ & $\mathrm{H} 17 \mathrm{~A}-\mathrm{C} 17-\mathrm{H} 17 \mathrm{C}$ & 109.5 \\
\hline $\mathrm{C} 9-\mathrm{C} 8-\mathrm{Cl} 8$ & $118.6(3)$ & $\mathrm{H} 17 \mathrm{~B}-\mathrm{C} 17-\mathrm{H} 17 \mathrm{C}$ & 109.5 \\
\hline $\mathrm{C} 8-\mathrm{C} 9-\mathrm{C} 9 \mathrm{~A}$ & $119.7(3)$ & $\mathrm{C} 26-\mathrm{C} 21-\mathrm{C} 22$ & $118.7(3)$ \\
\hline $\mathrm{C} 8-\mathrm{C} 9-\mathrm{H} 9$ & 120.1 & $\mathrm{C} 26-\mathrm{C} 21-\mathrm{C} 27$ & $121.2(3)$ \\
\hline $\mathrm{C} 9 \mathrm{~A}-\mathrm{C} 9-\mathrm{H} 9$ & 120.1 & $\mathrm{C} 22-\mathrm{C} 21-\mathrm{C} 27$ & $120.0(3)$ \\
\hline
\end{tabular}




\begin{tabular}{|c|c|c|c|}
\hline $\mathrm{C} 9-\mathrm{C} 9 \mathrm{~A}-\mathrm{N} 10$ & $116.6(3)$ & $\mathrm{O} 23-\mathrm{C} 22-\mathrm{C} 23$ & $118.4(4)$ \\
\hline $\mathrm{C} 9-\mathrm{C} 9 \mathrm{~A}-\mathrm{C} 5 \mathrm{~A}$ & $119.0(3)$ & $\mathrm{O} 23-\mathrm{C} 22-\mathrm{C} 21$ & $121.1(4)$ \\
\hline $\mathrm{N} 10-\mathrm{C} 9 \mathrm{~A}-\mathrm{C} 5 \mathrm{~A}$ & $124.1(3)$ & $\mathrm{C} 23-\mathrm{C} 22-\mathrm{C} 21$ & $120.5(4)$ \\
\hline $\mathrm{C} 11-\mathrm{N} 10-\mathrm{C} 9 \mathrm{~A}$ & $124.0(3)$ & $\mathrm{C} 22-\mathrm{C} 23-\mathrm{C} 24$ & $119.6(5)$ \\
\hline $\mathrm{N} 10-\mathrm{C} 11-\mathrm{N} 11$ & $117.2(3)$ & $\mathrm{C} 22-\mathrm{C} 23-\mathrm{H} 23 \mathrm{~A}$ & 120.2 \\
\hline $\mathrm{N} 10-\mathrm{C} 11-\mathrm{C} 11 \mathrm{~A}$ & $126.6(3)$ & $\mathrm{C} 24-\mathrm{C} 23-\mathrm{H} 23 \mathrm{~A}$ & 120.2 \\
\hline $\mathrm{N} 11-\mathrm{C} 11-\mathrm{C} 11 \mathrm{~A}$ & $115.8(3)$ & $\mathrm{C} 25-\mathrm{C} 24-\mathrm{C} 23$ & $120.9(4)$ \\
\hline $\mathrm{C} 1-\mathrm{C} 11 \mathrm{~A}-\mathrm{C} 4 \mathrm{~A}$ & $118.5(3)$ & $\mathrm{C} 25-\mathrm{C} 24-\mathrm{H} 24$ & 119.6 \\
\hline $\mathrm{C} 1-\mathrm{C} 11 \mathrm{~A}-\mathrm{C} 11$ & $120.3(3)$ & $\mathrm{C} 23-\mathrm{C} 24-\mathrm{H} 24$ & 119.6 \\
\hline $\mathrm{C} 4 \mathrm{~A}-\mathrm{C} 11 \mathrm{~A}-\mathrm{C} 11$ & $121.2(3)$ & $\mathrm{C} 24-\mathrm{C} 25-\mathrm{C} 26$ & $119.9(5)$ \\
\hline $\mathrm{C} 11-\mathrm{N} 11-\mathrm{C} 12$ & $118.3(3)$ & $\mathrm{C} 24-\mathrm{C} 25-\mathrm{H} 25$ & 120.0 \\
\hline $\mathrm{C} 11-\mathrm{N} 11-\mathrm{C} 16$ & $121.7(3)$ & $\mathrm{C} 26-\mathrm{C} 25-\mathrm{H} 25$ & 120.0 \\
\hline $\mathrm{C} 12-\mathrm{N} 11-\mathrm{C} 16$ & $111.0(2)$ & $\mathrm{C} 25-\mathrm{C} 26-\mathrm{C} 21$ & $120.5(4)$ \\
\hline $\mathrm{N} 11-\mathrm{C} 12-\mathrm{C} 13$ & $109.7(3)$ & $\mathrm{C} 25-\mathrm{C} 26-\mathrm{H} 26$ & 119.8 \\
\hline $\mathrm{N} 11-\mathrm{C} 12-\mathrm{H} 12 \mathrm{~A}$ & 109.7 & $\mathrm{C} 21-\mathrm{C} 26-\mathrm{H} 26$ & 119.8 \\
\hline $\mathrm{C} 13-\mathrm{C} 12-\mathrm{H} 12 \mathrm{~A}$ & 109.7 & $\mathrm{O} 21-\mathrm{C} 27-\mathrm{O} 22$ & $124.0(3)$ \\
\hline $\mathrm{N} 11-\mathrm{C} 12-\mathrm{H} 12 \mathrm{~B}$ & 109.7 & $\mathrm{O} 21-\mathrm{C} 27-\mathrm{C} 21$ & $118.3(3)$ \\
\hline $\mathrm{C} 13-\mathrm{C} 12-\mathrm{H} 12 \mathrm{~B}$ & 109.7 & $\mathrm{O} 22-\mathrm{C} 27-\mathrm{C} 21$ & $117.8(3)$ \\
\hline $\mathrm{H} 12 \mathrm{~A}-\mathrm{C} 12-\mathrm{H} 12 \mathrm{~B}$ & 108.2 & $\mathrm{C} 22-\mathrm{O} 23-\mathrm{H} 23$ & $96(5)$ \\
\hline $\mathrm{C} 11 \mathrm{~A}-\mathrm{C} 1-\mathrm{C} 2-\mathrm{C} 3$ & $-1.1(5)$ & $\mathrm{N} 11-\mathrm{C} 11-\mathrm{C} 11 \mathrm{~A}-\mathrm{C} 1$ & $32.9(4)$ \\
\hline $\mathrm{C} 1-\mathrm{C} 2-\mathrm{C} 3-\mathrm{C} 4$ & $-1.9(5)$ & $\mathrm{N} 10-\mathrm{C} 11-\mathrm{C} 11 \mathrm{~A}-\mathrm{C} 4 \mathrm{~A}$ & $38.8(4)$ \\
\hline $\mathrm{C} 2-\mathrm{C} 3-\mathrm{C} 4-\mathrm{C} 4 \mathrm{~A}$ & $2.8(5)$ & $\mathrm{N} 11-\mathrm{C} 11-\mathrm{C} 11 \mathrm{~A}-\mathrm{C} 4 \mathrm{~A}$ & $-148.7(3)$ \\
\hline $\mathrm{C} 3-\mathrm{C} 4-\mathrm{C} 4 \mathrm{~A}-\mathrm{C} 11 \mathrm{~A}$ & $-0.8(5)$ & $\mathrm{N} 10-\mathrm{C} 11-\mathrm{N} 11-\mathrm{C} 12$ & $5.5(4)$ \\
\hline $\mathrm{C} 3-\mathrm{C} 4-\mathrm{C} 4 \mathrm{~A}-\mathrm{N} 5$ & $178.1(3)$ & $\mathrm{C} 11 \mathrm{~A}-\mathrm{C} 11-\mathrm{N} 11-\mathrm{C} 12$ & $-167.7(3)$ \\
\hline $\mathrm{C} 4-\mathrm{C} 4 \mathrm{~A}-\mathrm{N} 5-\mathrm{C} 5 \mathrm{~A}$ & $115.3(3)$ & $\mathrm{N} 10-\mathrm{C} 11-\mathrm{N} 11-\mathrm{C} 16$ & $-138.6(3)$ \\
\hline $\mathrm{C} 11 \mathrm{~A}-\mathrm{C} 4 \mathrm{~A}-\mathrm{N} 5-\mathrm{C} 5 \mathrm{~A}$ & $-65.7(3)$ & $\mathrm{C} 11 \mathrm{~A}-\mathrm{C} 11-\mathrm{N} 11-\mathrm{C} 16$ & $48.2(4)$ \\
\hline $\mathrm{C} 4 \mathrm{~A}-\mathrm{N} 5-\mathrm{C} 5 \mathrm{~A}-\mathrm{C} 6$ & $-117.1(3)$ & $\mathrm{C} 11-\mathrm{N} 11-\mathrm{C} 12-\mathrm{C} 13$ & $151.8(3)$ \\
\hline $\mathrm{C} 4 \mathrm{~A}-\mathrm{N} 5-\mathrm{C} 5 \mathrm{~A}-\mathrm{C} 9 \mathrm{~A}$ & $62.6(4)$ & $\mathrm{C} 16-\mathrm{N} 11-\mathrm{C} 12-\mathrm{C} 13$ & $-60.6(3)$ \\
\hline $\mathrm{C} 9 \mathrm{~A}-\mathrm{C} 5 \mathrm{~A}-\mathrm{C} 6-\mathrm{C} 7$ & $-0.2(5)$ & $\mathrm{N} 11-\mathrm{C} 12-\mathrm{C} 13-\mathrm{N} 14$ & $56.6(4)$ \\
\hline $\mathrm{N} 5-\mathrm{C} 5 \mathrm{~A}-\mathrm{C} 6-\mathrm{C} 7$ & $179.6(3)$ & $\mathrm{C} 12-\mathrm{C} 13-\mathrm{N} 14-\mathrm{C} 17$ & $-177.1(3)$ \\
\hline $\mathrm{C} 5 \mathrm{~A}-\mathrm{C} 6-\mathrm{C} 7-\mathrm{C} 8$ & $-1.3(5)$ & $\mathrm{C} 12-\mathrm{C} 13-\mathrm{N} 14-\mathrm{C} 15$ & $-53.8(4)$ \\
\hline $\mathrm{C} 6-\mathrm{C} 7-\mathrm{C} 8-\mathrm{C} 9$ & $2.3(6)$ & $\mathrm{C} 17-\mathrm{N} 14-\mathrm{C} 15-\mathrm{C} 16$ & $177.3(3)$ \\
\hline $\mathrm{C} 6-\mathrm{C} 7-\mathrm{C} 8-\mathrm{Cl} 8$ & $-177.1(3)$ & $\mathrm{C} 13-\mathrm{N} 14-\mathrm{C} 15-\mathrm{C} 16$ & $53.8(4)$ \\
\hline $\mathrm{C} 7-\mathrm{C} 8-\mathrm{C} 9-\mathrm{C} 9 \mathrm{~A}$ & $-1.6(5)$ & $\mathrm{C} 11-\mathrm{N} 11-\mathrm{C} 16-\mathrm{C} 15$ & $-152.7(3)$ \\
\hline $\mathrm{C} 18-\mathrm{C} 8-\mathrm{C} 9-\mathrm{C} 9 \mathrm{~A}$ & $177.7(3)$ & $\mathrm{C} 12-\mathrm{N} 11-\mathrm{C} 16-\mathrm{C} 15$ & $60.9(4)$ \\
\hline $\mathrm{C} 8-\mathrm{C} 9-\mathrm{C} 9 \mathrm{~A}-\mathrm{N} 10$ & $174.2(3)$ & $\mathrm{N} 14-\mathrm{C} 15-\mathrm{C} 16-\mathrm{N} 11$ & $-56.8(4)$ \\
\hline $\mathrm{C} 8-\mathrm{C} 9-\mathrm{C} 9 \mathrm{~A}-\mathrm{C} 5 \mathrm{~A}$ & $0.0(5)$ & $\mathrm{C} 26-\mathrm{C} 21-\mathrm{C} 22-\mathrm{O} 23$ & $-179.2(4)$ \\
\hline $\mathrm{C} 6-\mathrm{C} 5 \mathrm{~A}-\mathrm{C} 9 \mathrm{~A}-\mathrm{C} 9$ & $0.8(5)$ & $\mathrm{C} 27-\mathrm{C} 21-\mathrm{C} 22-\mathrm{O} 23$ & $1.2(6)$ \\
\hline $\mathrm{N} 5-\mathrm{C} 5 \mathrm{~A}-\mathrm{C} 9 \mathrm{~A}-\mathrm{C} 9$ & $-178.9(3)$ & $\mathrm{C} 26-\mathrm{C} 21-\mathrm{C} 22-\mathrm{C} 23$ & $0.7(6)$ \\
\hline $\mathrm{C} 6-\mathrm{C} 5 \mathrm{~A}-\mathrm{C} 9 \mathrm{~A}-\mathrm{N} 10$ & $-172.9(3)$ & $\mathrm{C} 27-\mathrm{C} 21-\mathrm{C} 22-\mathrm{C} 23$ & $-178.9(4)$ \\
\hline $\mathrm{N} 5-\mathrm{C} 5 \mathrm{~A}-\mathrm{C} 9 \mathrm{~A}-\mathrm{N} 10$ & $7.4(5)$ & $\mathrm{O} 23-\mathrm{C} 22-\mathrm{C} 23-\mathrm{C} 24$ & $178.5(5)$ \\
\hline $\mathrm{C} 9-\mathrm{C} 9 \mathrm{~A}-\mathrm{N} 10-\mathrm{C} 11$ & $141.9(3)$ & $\mathrm{C} 21-\mathrm{C} 22-\mathrm{C} 23-\mathrm{C} 24$ & $-1.4(8)$ \\
\hline $\mathrm{C} 5 \mathrm{~A}-\mathrm{C} 9 \mathrm{~A}-\mathrm{N} 10-\mathrm{C} 11$ & $-44.2(5)$ & $\mathrm{C} 22-\mathrm{C} 23-\mathrm{C} 24-\mathrm{C} 25$ & $0.9(9)$ \\
\hline $\mathrm{C} 9 \mathrm{~A}-\mathrm{N} 10-\mathrm{C} 11-\mathrm{N} 11$ & $-171.4(3)$ & $\mathrm{C} 23-\mathrm{C} 24-\mathrm{C} 25-\mathrm{C} 26$ & $0.3(8)$ \\
\hline $\mathrm{C} 9 \mathrm{~A}-\mathrm{N} 10-\mathrm{C} 11-\mathrm{C} 11 \mathrm{~A}$ & $1.0(5)$ & $\mathrm{C} 24-\mathrm{C} 25-\mathrm{C} 26-\mathrm{C} 21$ & $-1.0(6)$ \\
\hline $\mathrm{C} 2-\mathrm{C} 1-\mathrm{C} 11 \mathrm{~A}-\mathrm{C} 4 \mathrm{~A}$ & $3.1(5)$ & $\mathrm{C} 22-\mathrm{C} 21-\mathrm{C} 26-\mathrm{C} 25$ & $0.5(5)$ \\
\hline $\mathrm{C} 2-\mathrm{C} 1-\mathrm{C} 11 \mathrm{~A}-\mathrm{C} 11$ & $-178.6(3)$ & $\mathrm{C} 27-\mathrm{C} 21-\mathrm{C} 26-\mathrm{C} 25$ & $-180.0(3)$ \\
\hline
\end{tabular}




$\begin{array}{llll}\mathrm{C} 4-\mathrm{C} 4 \mathrm{~A}-\mathrm{C} 11 \mathrm{~A}-\mathrm{C} 1 & -2.1(4) & \mathrm{C} 26-\mathrm{C} 21-\mathrm{C} 27-\mathrm{O} 21 & -178.5(3) \\ \mathrm{N} 5-\mathrm{C} 4 \mathrm{~A}-\mathrm{C} 11 \mathrm{~A}-\mathrm{C} 1 & 179.0(3) & \mathrm{C} 22-\mathrm{C} 21-\mathrm{C} 27-\mathrm{O} 21 & 1.1(5) \\ \mathrm{C} 4-\mathrm{C} 4 \mathrm{~A}-\mathrm{C} 11 \mathrm{~A}-\mathrm{C} 11 & 179.6(3) & \mathrm{C} 26-\mathrm{C} 21-\mathrm{C} 27-\mathrm{O} 22 & 1.7(4) \\ \mathrm{N} 5-\mathrm{C} 4 \mathrm{~A}-\mathrm{C} 11 \mathrm{~A}-\mathrm{C} 11 & 0.6(4) & \mathrm{C} 22-\mathrm{C} 21-\mathrm{C} 27-\mathrm{O} 22 & -178.7(3) \\ \mathrm{N} 10-\mathrm{C} 11-\mathrm{C} 11 \mathrm{~A}-\mathrm{C} 1 & -139.6(3) & & \end{array}$

Hydrogen-bond geometry $\left(A,{ }^{\circ}\right)$

$\mathrm{Cg} 1$ represents the centroid of the ring (C5A,C6-C9,C9A).

\begin{tabular}{lllll}
\hline$D-\mathrm{H} \cdots A$ & $D-\mathrm{H}$ & $\mathrm{H} \cdots A$ & $D \cdots A$ & $D-\mathrm{H} \cdots A$ \\
\hline $\mathrm{N} 14-\mathrm{H} 14 \cdots \mathrm{O} 22$ & $0.89(6)$ & $1.75(6)$ & $2.612(4)$ & $164(5)$ \\
$\mathrm{O} 23-\mathrm{H} 23 \cdots \mathrm{O} 21$ & $1.01(11)$ & $1.52(10)$ & $2.507(5)$ & $166(9)$ \\
$\mathrm{C} 4-\mathrm{H} 4 \cdots \mathrm{O} 22^{\mathrm{i}}$ & 0.95 & 2.33 & $3.261(4)$ & 166 \\
$\mathrm{C} 9-\mathrm{H} 9 \cdots \mathrm{O} 22^{\mathrm{ii}}$ & 0.95 & 2.25 & $3.202(4)$ & 176 \\
$\mathrm{C} 12-\mathrm{H} 12 B \cdots \mathrm{O} 21$ & 0.99 & 2.44 & $3.306(5)$ & 146 \\
$\mathrm{C} 15-\mathrm{H} 15 A \cdots \mathrm{N} 5^{\mathrm{ii}}$ & 0.99 & 2.56 & $3.539(4)$ & 170 \\
$\mathrm{C} 24-\mathrm{H} 24 \cdots \mathrm{Cg} 1^{\mathrm{iii}}$ & 0.95 & 2.83 & $3.637(5)$ & 144
\end{tabular}

Symmetry codes: (i) $x+1 / 2, y-1 / 2, z$; (ii) $x,-y+1, z+1 / 2$; (iii) $x-1 / 2, y+1 / 2, z-1$. 